Pontifícia Universidade Católica $_{\text {at }}$

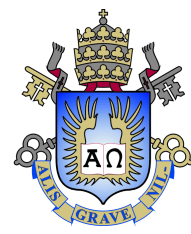

Antonio Capanema Guerra Galvão

\title{
Cascaded gamma-ray Counterpart of the IceCube Neutrinos
}

Dissertação de Mestrado

Dissertation presented to the Programa de Pós-graduação em Física of PUC-Rio in partial fulfillment of the requirements for the degree of Mestre em Física.

Advisor: Prof. Arman Esmaili Taklimi 


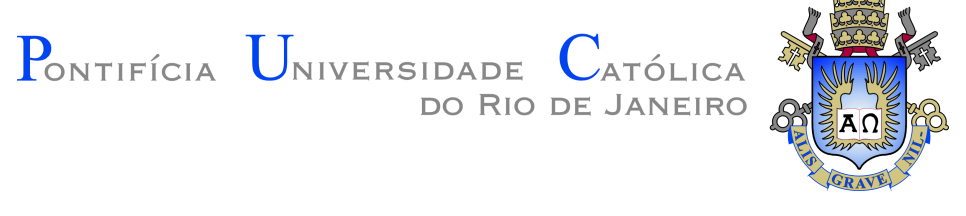

\title{
Antonio Capanema Guerra Galvão
}

\section{Cascaded gamma-ray Counterpart of the IceCube Neutrinos}

Dissertation presented to the Programa de Pós-graduação em Física of PUC-Rio in partial fulfillment of the requirements for the degree of Mestre em Física. Approved by the Examination Committee:

\author{
Prof. Arman Esmaili Taklimi \\ Advisor \\ Departamento de Física - PUC-Rio
}

Prof. Gero Arthur Hubertus Thilo Freiherr Von Gersdorff

Departamento de Física - PUC-Rio

Prof. Pasquale Dario Serpico

LAPTh

Rio de Janeiro, September 25th, 2020 
All rights reserved.

\section{Antonio Capanema Guerra Galvão}

The author obtained his Bachelor's Degree in Physics at the Pontifical Catholic University of Rio de Janeiro in 2018, where he is also earning his Master's Degree and is currently enrolled as a $\mathrm{PhD}$ student.

Bibliographic data

Galvão, Antonio Capanema Guerra

Cascaded gamma-ray Counterpart of the IceCube Neutrinos / Antonio Capanema Guerra Galvão; advisor: Arman Esmaili Taklimi. - 2020.

115 f: il. color. ; $30 \mathrm{~cm}$

Dissertação (mestrado) - Pontifícia Universidade Católica do Rio de Janeiro, Departamento de Física, 2020.

Inclui bibliografia

1. Física - Teses. 2. Astrofísica Multimensageira. 3. IceCube. 4. Neutrinos. 5. Raios Gama. 6. Cascata Eletromagnética. 7. Fundo Extragalático de Raios Gama.. I. Taklimi, Arman Esmaili. II. Pontifícia Universidade Católica do Rio de Janeiro. Departamento de Física. III. Título. 
To my professors, friends and family, for their continuous support and encouragement. 


\section{Acknowledgments}

I would like to first thank my advisor, Prof. Arman Esmaili, for his valuable comments on this dissertation and for his guidance during all these years. I admire him not only as a physicist, but also as a person, having taught me valuable lessons in both aspects. Arman is more than just an mentor: he is an inspiration and a friend which I hope to keep for the rest of my life. Thank you once again, I hope that this work can do justice to your mentoring and make you proud of your apprentice!

Then, I wish to extend my thanks to all professors who have closely followed my academic journey, both in and outside of PUC-Rio. Special thanks to Profs. Gero Gersdorff and Thiago Guerreiro for your lectures, your time and your trust.

Next, it is a difficult task to thank all of my friends as I have been lucky to have so many great people come and go throughout my life. To my friends from school, PUC-Rio, UC Berkeley and also from outside academia: thank you! I would also like to mention personally my dear friend Fatih Dinç, the nicest person I'll ever meet, for his constant support and advice during my career since 2016, and Luana Macedo, my girlfriend, for her love and emotional support during such a critical phase of my life. It is because of all of these amazing people that I am still fighting for my dreams with such passion and determination.

Thank you to CNPq and FAPERJ for the financial support of this work under the scholarships No. 157107/2018-3 and E-26/201.794/2019, respectively. This study was financed in part by the Coordenação de Aperfeiçoamento de Pessoal de Nível Superior - Brasil (CAPES) - Finance Code 001.

Last, but not least, thank you to my family, especially to my parents, who have raised me with so much care.

This dissertation is dedicated to all of you. 


\section{Abstract}

Galvão, Antonio Capanema Guerra; Taklimi, Arman Esmaili (Advisor). Cascaded gamma-ray Counterpart of the IceCube Neutrinos. Rio de Janeiro, 2020. 115p. Dissertação de Mestrado

- Departamento de Física, Pontifícia Universidade Católica do Rio de Janeiro.

In 2013, the IceCube Neutrino Observatory, located at the South Pole, discovered a flux of astrophysical neutrinos with $\mathrm{PeV}$ energies, later found to extend down to at least $\sim 10 \mathrm{TeV}$. Despite many efforts since then, determining their sources remains one of the most daunting challenges in the astrophysics community. In this dissertation, we investigate possible sources via a well-motivated multimessenger approach. In any production mechanism of cosmic neutrinos, there must also be a simultaneous production of $\gamma$-rays with comparable energies. Unlike neutrinos, which travel unscathed throughout the Universe, high energy $\gamma$-rays undergo interactions with background photons in an energy-degrading process known as "electromagnetic cascade". At the Earth, they constitute the diffuse extragalactic $\gamma$-ray background (EGB), precisely measured by the Fermi Gamma-ray Space Telescope in the $\mathrm{GeV}-\mathrm{TeV}$ range. By performing a conservative, quantitative, multimessenger analysis, we find a $\gtrsim 3 \sigma$ (possibly as large as $\sim 5 \sigma$ ) tension between IceCube and EGB data, pointing towards the existence of a new class of high energy cosmic accelerators, such as " $\gamma$-ray-opaque" sources.

\section{Keywords}

Multimessenger Astrophysics; IceCube; Neutrinos; $\gamma$-rays; Electromagnetic Cascade; Extragalactic $\gamma$-ray Background. 


\section{Resumo}

Galvão, Antonio Capanema Guerra; Taklimi, Arman Esmaili. Contrapartida em Raios-Gama Cascateados dos Neutrinos do IceCube. Rio de Janeiro, 2020. 115p. Dissertação de Mestrado Departamento de Física, Pontifícia Universidade Católica do Rio de Janeiro.

Em 2013, o IceCube Neutrino Observatory, localizado no Polo Sul, descobriu um fluxo de neutrinos astrofísicos com energias de PeV. Mais tarde, descobriu-se que este fluxo se estendia até pelo menos $\sim 10 \mathrm{TeV}$. Apesar de muitos esforços desde então, determinar as suas fontes permanece sendo um dos maiores desafios na comunidade de astrofísica. Nesta dissertação, investigamos possíveis fontes através de uma abordagem multimensageira bem motivada. Em qualquer mecanismo para a produção de neutrinos cósmicos, obrigatoriamente há produção simultânea de raios gama com energias comparáveis. Ao contrário de neutrinos, que atravessam o Universo ilesos, raios gama de altas energias sofrem interações com fótons de fundo em um processo de degradação de energia conhecido como "cascata eletromagnética". Na Terra, eles contituem o fundo extragalático de raios gama difuso (EGB), medido com precisão pelo Fermi Gamma-ray Space Telescope entre GeV-TeV . Realizando uma análise conservativa, quantitativa e multimensageira, encontra-se uma tensão de $\gtrsim 3 \sigma$ (possivelmente chegando a $\sim 5 \sigma$ ) entre os dados do IceCube e do EGB, apontando para a exitência de uma nova classe de aceleradores cósmicos de alta energia, como, por exemplo, fontes opacas para raios gama.

\section{Palavras-chave}

Astrofísica Multimensageira; IceCube; Neutrinos; Raios Gama; Cascata Eletromagnética; Fundo Extragalático de Raios Gama.. 


\section{Table of contents}

$\begin{array}{llr}1 & \text { Introduction } & 19\end{array}$

1.1 Multimessenger Astrophysics 20

1.2 IceCube Observations 23

$\begin{array}{lll}1.2 .1 & \text { Event Morphologies } & 26\end{array}$

1.3 Searches for Astrophysical Neutrino Sources 28

1.4 Objectives 32

2 Multimessenger Astrophysics $\quad 34$

2.1 Event Selection at IceCube 35

2.1.1 Atmospheric Backgrounds 35

$\begin{array}{lll}2.1 .2 & \text { IceCube Datasets } & 36\end{array}$

2.1.2.1 HESE Dataset $\quad 37$

2.1.2.2 Cascade Dataset 39

2.1.2.3 Through-going $\mu$-track Dataset 41

2.2 Emission of High-Energy Particles in Astrophysical Sources 43

2.2.1 $p p$ Scenario 44

$\begin{array}{lll}2.2 .2 & p \gamma \text { Scenario } & 45\end{array}$

2.2.3 $\nu-\gamma$ Connection 46

2.3 Neutrino Propagation 47

2.3.1 Decoherent Neutrino Oscillations 48

3 Electromagnetic Cascades $\quad 50$

$3.1 \gamma$ Backgrounds $\quad 51$

3.1.1 Cosmic Microwave Background 51

3.1.2 Extragalactic Background Light $\quad 52$

3.2 Physical Processes in $\gamma$-ray Propagation 54

3.2.1 Pair Production 55

3.2.2 Inverse Compton Scattering $\quad 56$

3.3 Cascade Regimes $\quad 59$

3.3.1 Leading Particle Regime $\quad 59$

$\begin{array}{lll}\text { 3.3.2 Multiplication Regime } & 60\end{array}$

$\begin{array}{lll}\text { 3.3.3 Low Energy Regime } & 60\end{array}$

3.4 Spectral Features of Cascaded $\gamma$-rays 61

3.5 Numerical Cascade Simulations 64

3.5.1 $\gamma$-Cascade Simulations $\quad 65$

3.5.2 Comparing Simulations with the Universal Spectrum 67

4 Methodology $\quad 69$

4.1 The Extragalactic $\gamma$-ray Background 69

$\begin{array}{ll}4.2 & \text { Multimessenger Analyses }\end{array}$

$\begin{array}{lll}4.2 .1 & \chi^{2} \text { Test } & 74\end{array}$

4.2.2 Integrated Flux above $50 \mathrm{GeV} \quad 77$

$\begin{array}{lll}4.2 .3 & \text { Spectrum Normalization } & 77\end{array}$

$\begin{array}{lll}4.2 .4 & \varepsilon_{\text {br Redshift }} & 78\end{array}$ 
$5 \quad$ Results and Discussions $\quad 80$

$5.1 \chi^{2}$ Analysis Results $\quad 80$

5.1.1 Limits in the $\varepsilon_{\mathrm{br}}-s_{h}$ Plane $\quad 80$

5.1.2 Limits in the $\Phi_{\text {astro }}-\gamma_{\text {astro }}$ Plane 84

5.2 Complementary Results and Discussions 85

$\begin{array}{lll}5.3 & \text { Interpretations and Discussions } & 87\end{array}$

$\begin{array}{llr}6 & \text { Final Remarks } & 89\end{array}$

$\begin{array}{lr}\text { Bibliography } & 91\end{array}$

$\begin{array}{lll}\text { A A Brief Review on Cosmology } & 108\end{array}$

B SFR and other Source Population Distributions 113 


\section{List of figures}

Figure 1.1 The opacity of the universe as a function of redshift. The gray points indicate the highest energy $\gamma$-ray coming from each blazar analyzed. The curves shown represent the cosmic horizon $^{1}$ for three different EBL (target photon distribution) models. Figure reproduced from [2].

Figure 1.2 Schematic representation of different messengers propagating through the Universe. Neutrinos offer a direct, uninterrupted path towards its source.

Figure 1.3 Schematic view of IceCube's 86-string hexagonal array extending to a depth of $\sim 2500 \mathrm{~m}$. Figure reproduced from [13].

Figure 1.4 Arrival directions of the highest energy IceCube events in equatorial coordinates. Different event morphologies (see Section 1.2.1) are represented by differently colored symbols. The gray line indicates the Galactic plane. Figure reproduced from [19].

Figure 1.5 Flavor composition of astrophysical neutrinos at the Earth (i.e. measured by IceCube, expressed in terms of likelihood variation $-2 \Delta \ln L$; left) and inferred production ratio at their sources (right). Every point in this "flavor triangle" represents a possible flavor composition $\left(\nu_{e}: \nu_{\mu}: \nu_{\tau}\right)$ (no distinction is made between neutrinos and antineutrinos) at the Earth, " $\oplus$ ", and at the source, " $S$ ". Several C.L. contours are labelled in white and gray solid lines. Three benchmark production scenarios (see Section 2.2) and their $3 \sigma$ ranges are shown as red/orange/green points and contours, respectively. The resulting compositions at the Earth are obtained after simulating decoherent neutrino oscillations (see Section 2.3.1). Figure reproduced from [20].

Figure 1.6 A cascade $^{2}$ (left, $E_{\nu}=1.14 \mathrm{PeV}$ ) and a $\mu$-track (right, $E_{\nu}=880 \mathrm{TeV}$ ) at the IceCube detector: two astrophysical neutrino events with distinct morphologies. DOMs are represented by spheres. Their size indicates the amount of Cherenkov light collected by each DOM. Color represents time: in the track signature, the muon travels from right to left. These (and many more) pictures can be found in the official IceCube website [29]. (1.6(a)) Cascade (1.6(b)) $\mu$-track 
Figure 2.1 Number of events per deposited energy after 2 years [15] (2.1(a)), 3 years [18] $(2.1(\mathrm{~b})), 4$ years [87] $(2.1(\mathrm{c}))$ and 6 years [88] (2.1(d)) of HESE data. Expected backgrounds due to atmospheric muons and neutrinos, as well as their uncertainties are indicated in the red, blue and hatched regions. Error bars in the data points represent a $1 \sigma$ uncertainty. A total of 28 astrophysical neutrino events were reported in the 2year sample: an excess with respect to the expected background events at $4.1 \sigma$ confidence level (C.L.). This was followed by 37 candidate events in the 3-year sample, 54 events after 4 years and 82 in the 6-year HESE sample. The gray shaded region to the left of the vertical line in the 6-year plot marks where $E_{\nu}<60 \mathrm{TeV}$, which has been excluded from this and all previous analyses (see text).

(2.1(a)) HESE 2yr 38

(2.1(b)) HESE 3yr 38

(2.1(c)) HESE 4yr 38

(2.1(d)) HESE 6yr 38

Figure 2.2 Most recent results for deposited energies in HESE, after 7.5 years of data [89]. Data points are black crosses with $1 \sigma$ error bars, and the colored stacked histogram shows the best fit expectations for each component of the total neutrino flux: astrophysical (yellow), conventional atmospheric neutrinos (red) and atmospheric muons (blue). Just as before, events below 60 $\mathrm{TeV}$ (shaded region) are excluded from the analysis/fit, leaving us with 60 events above this threshold.

Figure 2.3 Measured astrophysical neutrino flux with $1 \sigma$ error bars for IceCube's cascade dataset after 2 years [90] (2.3(a)) and 4 years [91] $(2.3(\mathrm{~b}))$ of data-taking. These data points were obtained using a differential model, where an $E^{-2}$ weight was assumed in each energy bin. Best-fit power-laws are represented by dashed lines with a $\pm 1 \sigma$ red band around them. In Figure 2.3(b), the dark red shaded region represents the sensitive energy range (i.e. the energy range included in the power-law fit). 40 (2.3(a)) Cascade 2yr 40 (2.3(b)) Cascade 4yr

Figure 2.4 Astrophysical neutrino flux with 6 years of cascade events [92]. Crosses represent the differential model best fit results (see caption in Fig. 2.3) and solid (dashed) colored lines represent several flux models in (outside of) the sensitive energy range of $16 \mathrm{TeV}<E_{\nu}<2.6 \mathrm{PeV}$. The red line is the one we are interested in, with a single power-law fit and a $1 \sigma$ red error band around it. 40

Figure 2.5 Best-fit neutrino spectra considering through-going $\mu$ tracks coming from the northern hemisphere after 6 years [94] (2.5(a)) and 8 years [17] (2.5(b)) of collecting data. Expected conventional and (upper bounds for) prompt neutrino contributions are shown in blue and green, respectively. 
Figure 2.6 Best-fit neutrino spectra with 9.5 years of throughgoing $\mu$-track data [95]. The sum of the astrophysical (red line) and conventional (purple line) components provide a fit to the experimental data. The prompt atmospheric component (green line) is not shown because its best-fit normalization is zero.

Figure 2.7 Single power-law astrophysical neutrino flux parameters for each IceCube dataset, including their best-fit values and $1 \sigma$ contour around them. The contours which are of interest to us are the HESE (green), Tracks (blue) and Cascades assuming $p \gamma$ production mechanism (yellow). ${ }^{3}$ See Section 2.2 for further details on the production mechanisms. Figure reproduced from [92].

Figure 3.1 EBL intensity at $z=0$ as a function of wavelength as modelled by Domínguez et al [115]. The solid black line represents their EBL best fit with uncertainty given by the shaded gray region. Blue dot-dashed and red dashed lines correspond to other EBL models found in literature ([117] and [118] respectively). Colored solid lines are upper bounds from the study of blazars. Empty symbols are direct measurements while filled symbols are from galaxy counts, usually considered as lower limits. 53

Figure 3.2 EBL evolution with redshift (top left panel: $z=0.2$ ), reproduced from [115]. The solid and dashed black lines represent their model under different assumptions for $z>1$ extrapolation (see the reference for details). Red dashed, blue dot-dashed, green dotted and gray dot-long-dashed lines represent contributions from quiescent, star-forming galaxies, starbursts and AGN, respectively. Magenta dashed and orange dot-dashed lines are from other models ([117] and [118] respectively), for comparison. 54

Figure 3.3 Illustrative representation of an electromagnetic cascade, adapted from [107]. The standard notation of curly lines for photons and straight solid lines for electrons is adopted. From left to right, the vertical dashed lines separate the leading particle, multiplication and low energy regimes. In the leading particle regime, examples of non-leading electrons which start new cascades at $E>E_{t h, c m b}$ and $E_{t h, c m b}>E>E_{t h, e b l}$ are shown. In the multiplication regime, the uppermost photon is an example of a photon produced with energy $E_{t h, e b l}>E_{\gamma}>E_{X}$, becoming cascade-sterile since it cannot pair produce.

Figure 3.4 Cascaded fluxes (blue lines) above $E_{X} \approx 100 \mathrm{MeV}$ from point sources located at different redshifts. The black dashed line corresponds to the cutoff energy $E_{t h, e b l}(z)$ predicted by the universal spectrum in Eq. 3-27. Dashed grey lines correspond to $E_{-2}$ (as predicted by the Eq. $3-27$ ) and $E^{-1.9}$ spectra. The latter index seems to fit the simulations better for $z \lesssim 0.2$. 
Figure 4.1 EGB flux measured by The Fermi-LAT Collaboration between $100 \mathrm{MeV}$ and $820 \mathrm{GeV}$ for three different foreground models (see Table 4.1) at high latitudes, $|b|>20^{\circ}$ [126]. Error bars include statistical and systematic uncertainties for each foreground model added in quadrature, while the yellow shaded region represents the systematic uncertainty coming specifically from modelling the diffuse galactic emission. For foreground model A, the cumulative intensity solely from resolved sources is shown as the gray band.

Figure 4.2 EGB data (red points) together with its three conventional contributions considered in this work (blazars: blue band [129], RGs: black striped band [130], SFGs: gray band [131]) and their respective uncertainties. Their sum is represented by the yellow band. Pink lines pertain to the original paper's [129] intention to set constraints on the DM annihilation cross section and are unrelated to this thesis.

Figure 4.3 Left: same as Figure 4.1 but for the IGRB [126]. Right: Conventional/well-established contributions to the IGRB, together with their sum and total uncertainty represented by the blue band [141].

Figure 4.4 Cosmological redshift of the break energy in our injection spectrum 4-1. As expected, this effect results in $E_{\mathrm{br}}<\varepsilon_{\mathrm{br}}$ since all colored lines fall below the dashed gray line (which indicates no redshift).

Figure 5.1 Limits in the $\varepsilon_{\mathrm{br}}-s_{h}$ plane from IceCube (gray contours, corresponding to $E_{\mathrm{br}}=E_{\mathrm{th}}$ ) and EGB data (colored contours, at $90 \% \mathrm{CL}$ corresponding to $\Delta \chi^{2}=4.61$ ), with arrows pointing towards the allowed regions. EGB constraints are obtained assuming the smallest (lower contours) and largest (upper contours) normalizations allowed by IceCube at $1 \sigma$ for each $s_{h}$, according to Figure 2.7, for each dataset (9.5y Tracks: blue, 6y Cascades: green, 7.5y HESE: orange). A clear tension can be seen between neutrino and $\gamma$-ray data in the Cascade $s_{h}$ range. Figure reproduced from [79].

Figure 5.2 Neutrino (black lines), cascaded (red lines) and total (conventional + cascaded, blue lines) $\gamma$-ray fluxes for specific $\left(\varepsilon_{\mathrm{br}}, s_{h}\right)$ values, compared with the IceCube allowed regions for each dataset (black dashed lines) and the Fermi EGB data (red data points). For each flux, the darker tone has normalization $\Phi_{\max }$ while the lighter tone has normalization $\Phi_{\min }$, with a filling of the same color between both lines. $\mathcal{A}_{\text {min }}$ and $\mathcal{A}_{\text {max }}$ are the $\mathcal{A}$ values obtained from the minimization of our $\chi^{2}$ function $4-5$. 
Figure 5.3 Limits for fixed $E_{\mathrm{br}}$ values in the $\Phi_{\text {astro }} s_{h}$ plane are given by black contours, with arrows pointing towards allowed regions. The blue, green and orange contours are the $1 \sigma$ (dashed) and $2 \sigma$ (dotted) allowed regions for the 9.5y Tracks, 6y Cascade and 7.5y HESE IceCube datasets, respectively, with stars representing their best-fit values in Eq. 4-8. Since, $E_{\mathrm{th}}=16 \mathrm{TeV}$ for the Cascade dataset, by assuming a change in spectral behaviour at $E_{\mathrm{br}}=10 \mathrm{TeV}$, we find a $\gtrsim 3 \sigma$ tension. This tension increases to $\sim 5 \sigma$ for $E_{\mathrm{br}}=1 \mathrm{TeV}$. Figure adapted from [79].

Figure 5.4 Exclusions from cascaded $\gamma$-ray flux integration (colored contours) as described in Section 4.2.2 for $q=82 \%, 86 \%$ and $88 \%$, with arrows pointing towards allowed regions. Contours for smallest(largest) normalization allowed at $1 \sigma$ CL are given by dashed(solid) colored lines. Gray contours indicate IceCube's thresholds for each dataset $\left(E_{\mathrm{br}}=E_{\mathrm{th}}\right)$. Similar to Figure 5.1, we see a multimessenger tension arising from the Cascade dataset. Figure reproduced from [79].

Figure B.1 Redshift dependence of the cosmic SFR density. The fit from Eq. B-1 is shown by the orange line. The gray points and dotted line are the compiled data and fit from [186]. The colored points are more recent high- $z$ data from this Figure's source article [188] and references therein.

Figure B.2 Comparison between the SFR [188] and BL Lac [189] evolution functions normalized to unity at $z=0$. The BL Lac function has been parametrized using a luminosity-dependent density evolution model. Figure reproduced from [80]. 


\section{List of tables}

Table 1.1 IceCube's main signatures and their corresponding $\nu$ interactions.

Table 2.1 Best-fit parameters and low-energy threshold energies for each IceCube dataset considered in this thesis. The $\Phi_{\text {astro }}$ reported values are per-flavor. In order to obtain each dataset's $\Phi_{\text {astro }}$, IceCube assumes a $(1: 1: 1)$ flavor ratio at the Earth and uses this to extrapolate the normalization of all astrophysical neutrinos from the events of an individual dataset.

Table 2.2 Best-fit parameter values for the PMNS matrix following the parametrization in Eq. 2-15. The fit assumes normal ordering for the neutrino masses $\left(m_{1}<m_{2}<m_{3}\right)$ and includes data from solar, reactor, accelerator and atmospheric (including Super-Kamiokande) neutrino experiments [102, 103].

Table 3.1 Summary of PP, ICS and regular Compton scattering cross sections and order-of-magnitude mean free paths for different incoming particle energies, adapted from [107]. The target photons come from either the COB (optical part of the EBL) or the CMB. Their respective densities are also displayed in the table. The mean free path for regular Compton scattering is shown for comparison.

Table 4.1 Main differences between the three FG models for the DGE employed in Fermi's EGB analysis [126].

Table 4.2 EGB intensities and upper uncertainties, in $\left[\mathrm{cm}^{-2} \mathrm{~s}^{-1}\right.$ $\mathrm{sr}^{-1}$, for FG model A, which is the one used in this work. The uncertainties include the statistical, systematic and foreground modeling errors added in quadrature [126].

Table 4.3 Values of $q$ such that $\Delta \chi^{2}>q$ implies an exclusion of a scenario at a specific confidence level (CL). The values are reported for $m=1,2$ and 3 free parameters. This table is based on Table 40.2 from Particle Data Group's 2020 Statistics review [149].

Table A.1 Energy density dependence on the scale factor following $\rho_{i}=\rho_{i, 0} a^{-n_{i}}$ for different components of our universe. 


\section{List of Abreviations}

AGN - Active Galactic Nucleus/Nuclei

BAO - Baryon Acoustic Oscillations

BSM - Beyond the Standard Model

CC - Charged Current

CIB - Cosmic Infrared Background

CMB - Cosmic Microwave Background

COB - Cosmic Optical Background

DGE - Diffuse Galactic Emission

DM - Dark Matter

DOM - Digital Optical Module

EBL - Extragalactic Background Light

EGB - Extragalactic Gamma-ray Background

FLRW - Friedmann-Lemaître-Robertson-Walker

GR - General Relativity

GRB - Gamma-Ray Burst

GW - Gravitational Wave

GZK - Greisen-Zatsepin-Kuzmin

HESE - High Energy Starting Events

HST - Hubble Space Telescope

IGRB - Isotropic diffuse Gamma-Ray Background

IMF - Initial Mass Function

LAT - Large Area Telescope

LF - Luminosity Function

LIGO - Laser Interferometer Gravitational-Wave Observatory

NC - Neutral Current 
PWN - Pulsar Wind Nebula

QED - Quantum Electrodynamics

RG - Radio Galaxy

SBG - Starburst Galaxy

SFG - Star-Forming Galaxy

SFR - Star-Formation Rate

SMBH - Supermassive Black Hole

SNR - Supernova Remnant

TDE - Tidal Disruption Event

(UHE)CR - (Ultra-High Energy) Cosmic Ray 
If there is no point in the universe that we discover by the methods of science, there is a point that we can give the universe by the way we live, by loving each other, by discovering things about nature, by creating works of art. - Steven Weinberg

Frankenberry, The Faith of Scientists: In Their Own Words. 


\section{Introduction}

Astrophysics is a truly vast field which studies the nature of astronomical objects using tools from most, if not all, branches of physics, from classical to quantum mechanics, from general relativity to particle physics. The Universe is home to a countless variety of such objects, providing us with plenty of subjects to test and improve our theories. Unfortunately for us, however, we are merely spectators of the cosmos, confined to a tiny speck of land we call Earth, awaiting for extraterrestrial signals which are far from being under our control. These signals come in the form of cosmic "messengers": particles travelling long distances and carrying information about their sources, leaving us with the task of interpretation. There are four main messengers currently studied by astrophysicists: cosmic rays (CRs), photons, neutrinos and gravitational waves (GWs). Separately, each messenger gives us a unique view of the Universe, but together, they complement each other in incredible ways. This coordinated observation and interpretation of different messenger signals is the idea behind multimessenger astrophysics, which is the central theme of this thesis.

This thesis is divided into six chapters. The goal of this first chapter is to briefly introduce the field of multimessenger astronomy, focusing primarily on the astrophysical neutrino flux observations by the IceCube Neutrino Observatory (or simply, IceCube). The (yet unanswered) question we want to tackle is simple: what are the sources of the IceCube neutrinos? In particular, what information can we extract about them using a multimessenger approach? After outlining the main efforts from the community to try and solve this puzzle, we move on to Chapter 2, where we present all necessary details regarding IceCube's results. This is followed by a comprehensive description of high-energy neutrino and $\gamma$-ray production in dense astrophysical environments and their propagation through the Universe, establishing a multimessenger connection between both. Neutrinos travel essentially undisturbed, while $\gamma$-rays undergo multiple interactions, making their propagation considerably trickier to follow. Chapter 3 is solely dedicated to understanding this process in great detail. Chapter 4 begins by presenting the $\gamma$-ray data collected by the Fermi Gammaray Space Telescope (Fermi, for short). Having all the foundations laid out, the remainder of Chapter 4 aims to thoroughly describe our analyses methods. 
We report, interpret and discuss our results in Chapter 5. Finally, in Chapter 6 , we conclude by summarizing our results and pointing our what we can learn from them.

Before we go any further, I would like to stress that this thesis is not just meant to be a presentation of new results, but also a helpful introduction to newcomers in astrophysics. This means that we will build our knowledge from scratch, assuming the reader is familiar with the essentials of particle physics and general relativity. A useful review on cosmology can be found in Appendix A, with the most relevant derivations and formulae necessary for the main text (especially for Chapter 2). We shall be using natural units $c=\hbar=1$ throughout this thesis unless specified.

\section{1}

\section{Multimessenger Astrophysics}

Astroparticle physics is and has been historically a multi-frequency and multimessenger science by nature. From the early 20th century onward, we have observed the sky using light from different regions of the electromagnetic spectrum, ranging from radio waves to $\gamma$-rays, allowing us to map the distributions of stars and galaxies in the Universe, predict their temperatures, chemical compositions and much more. Since their discovery in 1912 by Victor Hess [1], we have found that CRs themselves constitute a diverse class of charged messengers, including protons, positrons, electrons and heavy nuclei. Recently, the emergence of neutrino and GW astronomy has marked the so-called "dawn of the multimessenger era", where we can hope to study astrophysical objects by combining multiple different particle observations.

Naturally, each messenger has its unique properties which allow us to probe different aspects of their sources (and the space between them and us). No messenger alone can give us the complete picture. Due to their charge, CRs are susceptible to plasma shock acceleration and magnetic field deflection, making them a great tool for understanding acceleration mechanisms in dense astrophysical environments. However, this deflection comes with the loss of directional information, making it impossible (or extremely hard) to trace back to their sources.

Although photons only travel from the surface of optically thick sources, they do move in a straight line (ignoring gravitational lensing effects), making them suitable for astronomy. The complications arise at high energies. $\gamma$-rays with $100 \mathrm{GeV}$ (which play a crucial role in this thesis) run the risk of interacting with low-energy target photons in space via pair production. This probability increases with energy to the degree that the photon attenuation 
length for $\sim \mathrm{PeV}$ photons reaches Galactic scales. A study done by the FermiLAT collaboration confirms the existence of a cosmic horizon for high-energy $\gamma$-rays [2]. Their work consisted in collecting data from 739 blazars over 9 years of operations, and recording the highest-energy photons detected from each one, displayed as gray points in Figure 1.1. The plot shows us, for example, that $>\mathrm{TeV} \gamma$-rays cannot reach us if emitted any further than $z \sim 0.1$.

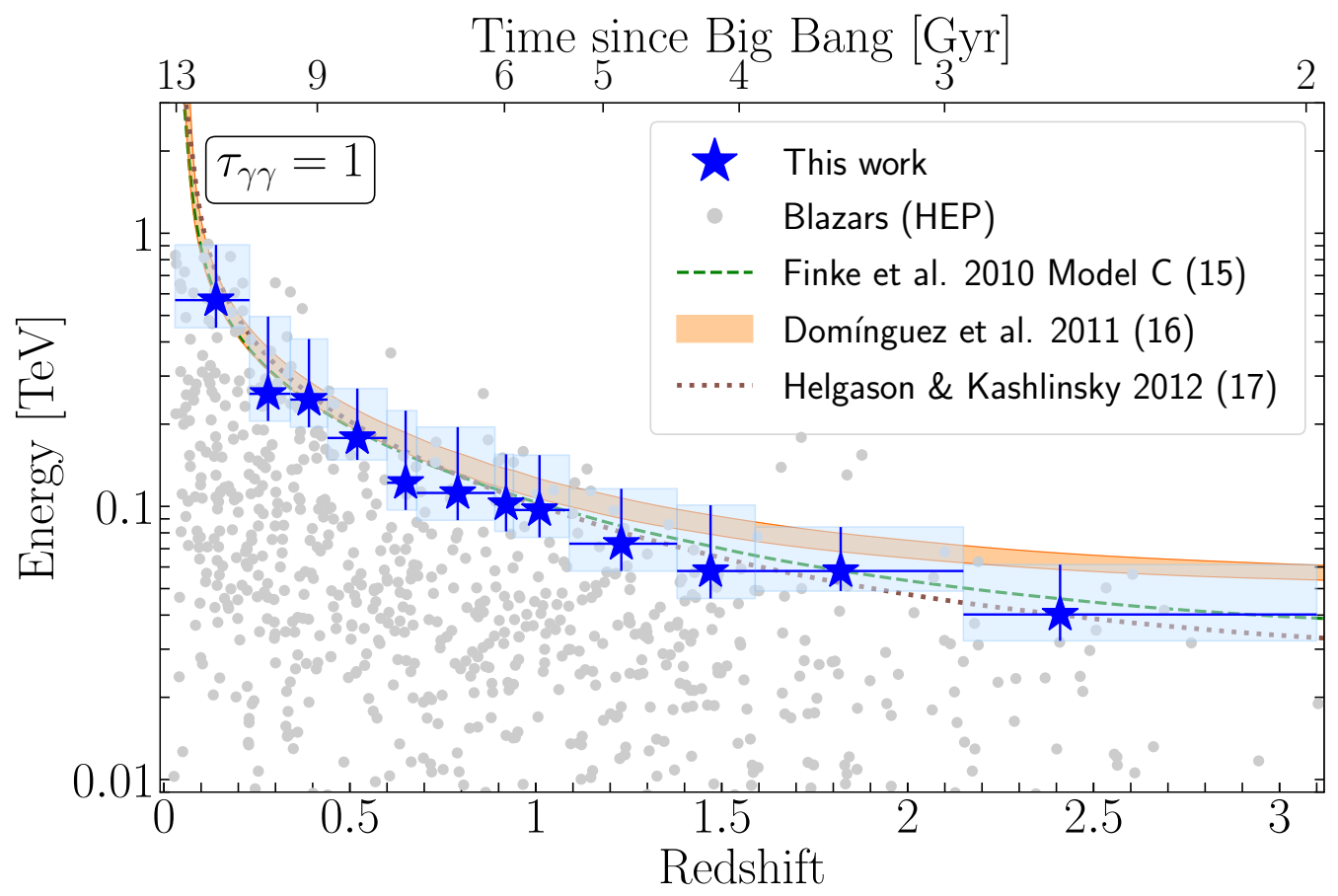

Figure 1.1: The opacity of the universe as a function of redshift. The gray points indicate the highest energy $\gamma$-ray coming from each blazar analyzed. The curves shown represent the cosmic horizon ${ }^{1}$ for three different EBL (target photon distribution) models. Figure reproduced from [2].

Although the initial $\gamma$-ray is lost after pair production, the resulting electron (or positron) can undergo inverse Compton scattering with another background photon, producing a new $\gamma$-ray with slightly lower energy than the original one. This pair production $\rightarrow$ inverse Compton scattering chain is repeated in a process called "electromagnetic cascade" (the main theme of Chapter 3).

The electromagnetic cascade (or simply "cascade") was first proposed in the late 1960s, soon after the discovery of the cosmic microwave background [3] and of the Greisen-Zatsepin-Kuzmin (GZK) cutoff $[4,5,6]$, and its first applications came in deriving an upper limit to the cosmogenic neutrino flux

\footnotetext{
${ }^{1}$ This horizon is defined as the distance/redshift where the observed intensity of $\gamma$ rays decreases by $1 / \mathrm{e}$ with respect to the initial intensity. In mathematical terms, $I_{\mathrm{obs}}=$ $I_{\text {init }} \times \mathrm{e}^{-\tau_{\gamma \gamma}(E, z)}$, where $\tau_{\gamma \gamma}$ is the optical depth. $\tau_{\gamma \gamma}=1$ determines the position of the horizon, beyond where the medium is optically thick to photons.
} 
(neutrinos produced in the GZK process [7]) [8, 9]. Intergalactic magnetic fields are weak enough $\left(B \gtrsim 10^{-19} \mathrm{G}\right.$ for a field coherence length of $L_{B} \geq 1 \mathrm{Mpc}$ is a lower limit [10]) that this cascading process does not deflect electrons and positrons significantly for us to lose directionality, although an extended " $\gamma$-ray halo" could form around the source due to $e^{ \pm}$deflection (yet to be observed) [11].

Neutrinos as cosmic messengers are radically different from the first two. Weak interactions are the only way of stopping neutrinos (they also experience the gravitational force, although we can safely neglect it here $)^{2}$. Luckily, their extremely small cross sections allow them to easily escape their sources and travel undisturbed through the Universe, making them completely reliable for directional searches and studying production mechanisms. But this also makes them rather difficult to detect, requiring huge volumes of dense material to provoke an interaction. Astrophysical neutrino detection at IceCube is promptly reviewed in the next section. Figure 1.2 summarizes the main points in this section: a single source produces multiple messengers; CRs deflect from their original trajectories and $\gamma$-rays get absorbed while neutrinos are exempt from these setbacks.

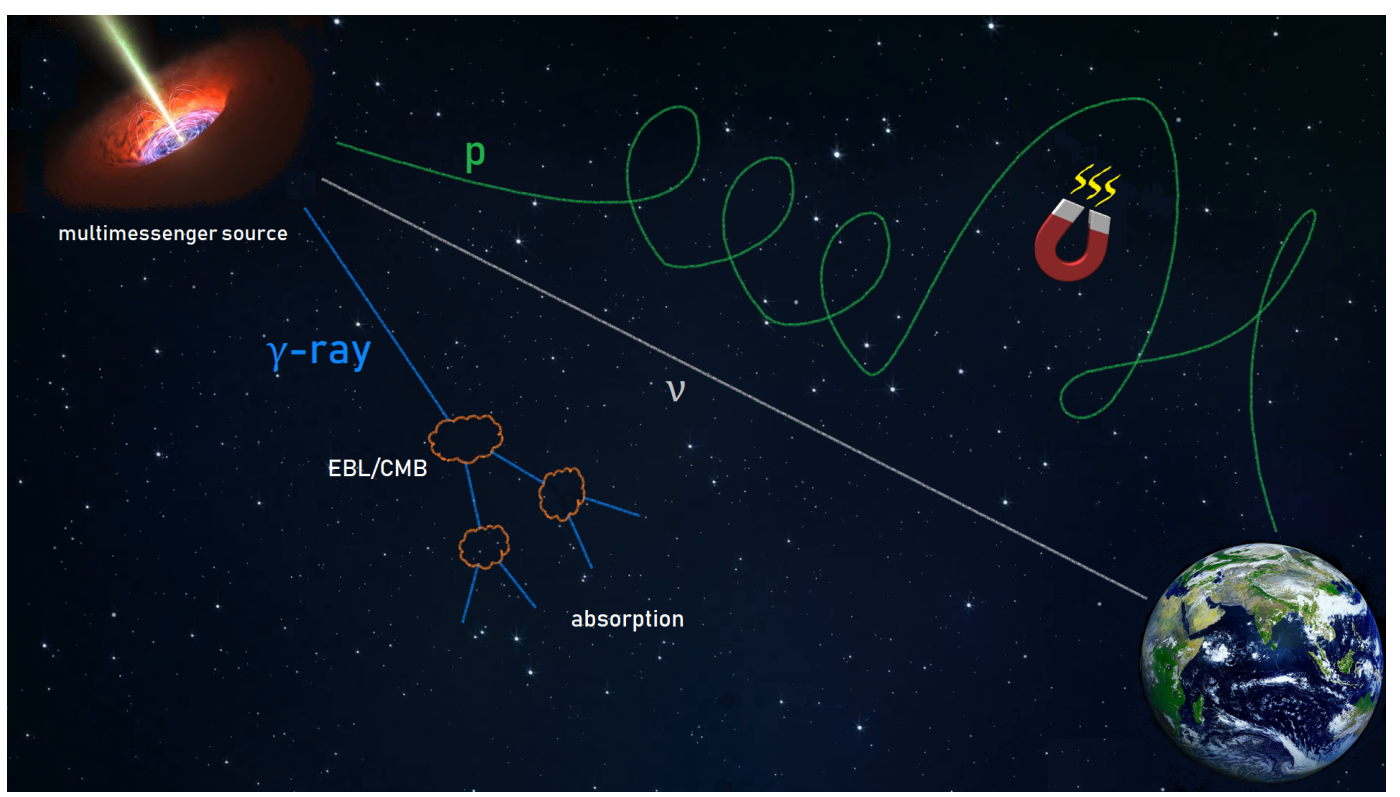

Figure 1.2: Schematic representation of different messengers propagating through the Universe. Neutrinos offer a direct, uninterrupted path towards its source.

${ }^{2}$ Neutrinos are effectively massless in all our considerations (they travel practically at the speed of light), except when discussing their flavor oscillations in Section 2.3.1. 
On a quick note, gravitational wave astronomy has shown a lot of promise since its "inauguration" after the first GW observation by the Laser Interferometer Gravitational-Wave Observatory (LIGO) and Virgo collaborations in February 2016 [12]. GWs propagate freely at the speed of light and reveal to us important information on the environments where nonthermal particle production might occur (e.g. binary black hole or neutron star mergers). Nevertheless, it is still a rudimentary field with incredibly challenging detection requirements, which we will not be discussing further in this work.

\section{2}

\section{IceCube Observations}

IceCube is a neutrino observatory located at the South Pole dedicated to searching for astrophysical neutrinos with energies $\gtrsim 100 \mathrm{GeV}$. It is comprised of 86 strings carrying 60 digital optical modules (DOMs) each, which have been deployed deep within the Antarctic ice. The DOMs are distributed in a 3D hexagonal array spanning a fiducial volume of $1 \mathrm{~km}^{3}$ of ice (see Section 2.1.2.1 for the definition of "fiducial volume"), with a densely instrumented central region called DeepCore optimized for detecting neutrinos with a few tens of $\mathrm{GeV}$. More information about the detector's arrangement can be found in Figure 1.3 and Ref. [13].

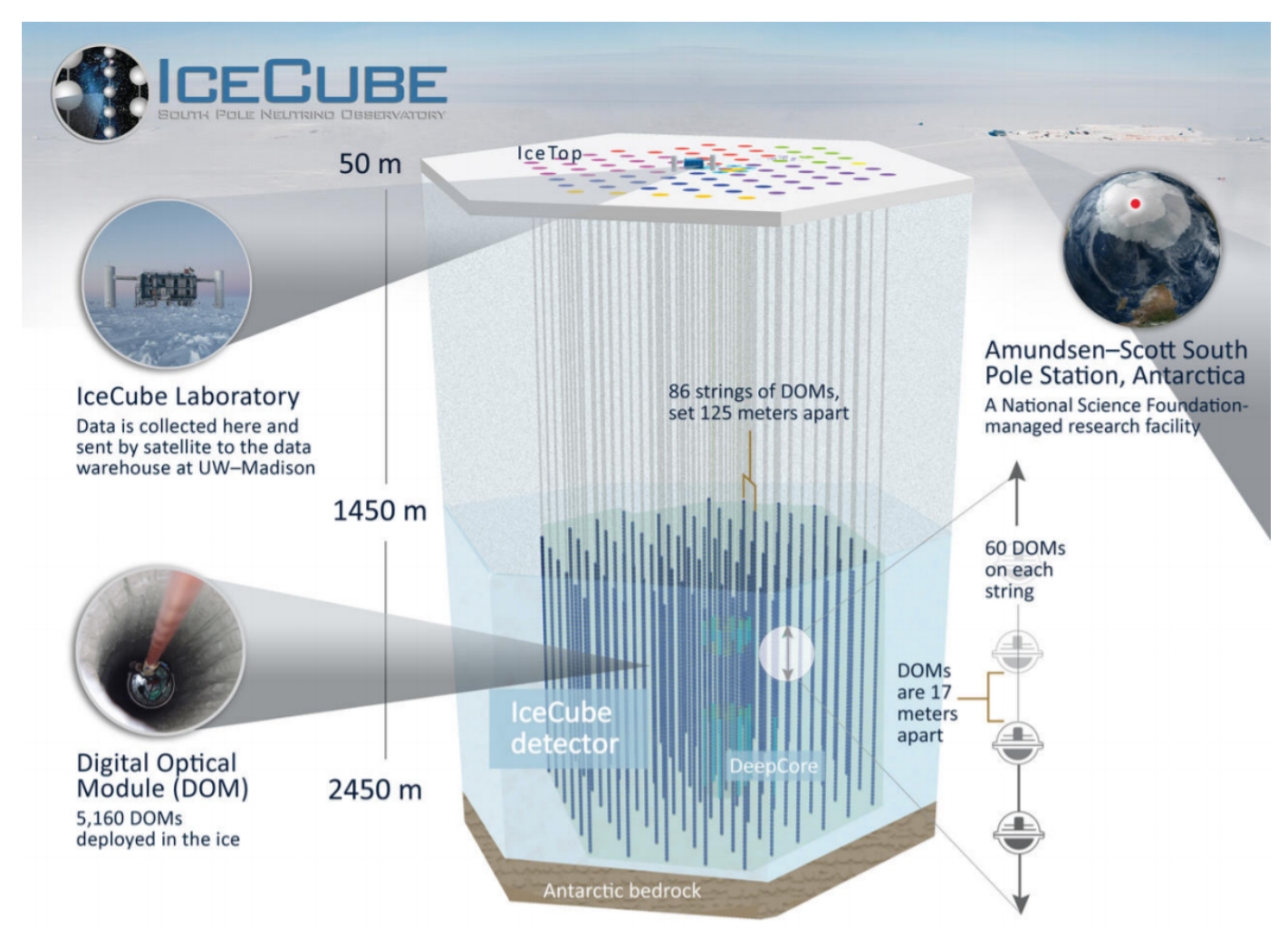

Figure 1.3: Schematic view of IceCube's 86-string hexagonal array extending to a depth of $\sim 2500 \mathrm{~m}$. Figure reproduced from [13]. 
Since its completion and inauguration in December 2010, IceCube has reported the observation of astrophysical neutrinos with energies between $\sim 10$ $\mathrm{TeV}$ and $\sim 6 \mathrm{PeV}$. In 2013, the collaboration released its first direct evidence for their existence [14] with the observation of two $\sim \mathrm{PeV}$-energy neutrinos and a background-only ${ }^{3}$ hypothesis rejected at $2.8 \sigma$ confidence level (C.L.). Soon after, 26 additional events were reported [15] by improving their sensitivity and extending their energy coverage down to $\sim 30 \mathrm{TeV}$, increasing the confidence in an astrophysical flux interpretation to $\sim 4 \sigma$. Until then, only events with interaction vertex located inside the fiducial volume of the detector were considered. However, IceCube eventually extended their searches and included signals starting outside the detector [16], increasing the statistical significance of an astrophysical excess to $6.7 \sigma$ [17]. The overall data are consistent with (quasi-) isotropic arrival directions (with a mild tendency towards the Galactic center, see Figure 1.4) and an equal flux of all three neutrino flavors (see Figure 1.5), the former suggesting that they must have an extragalactic origin [18].

\section{Through-going tracks (>200 TeV) Cascades}

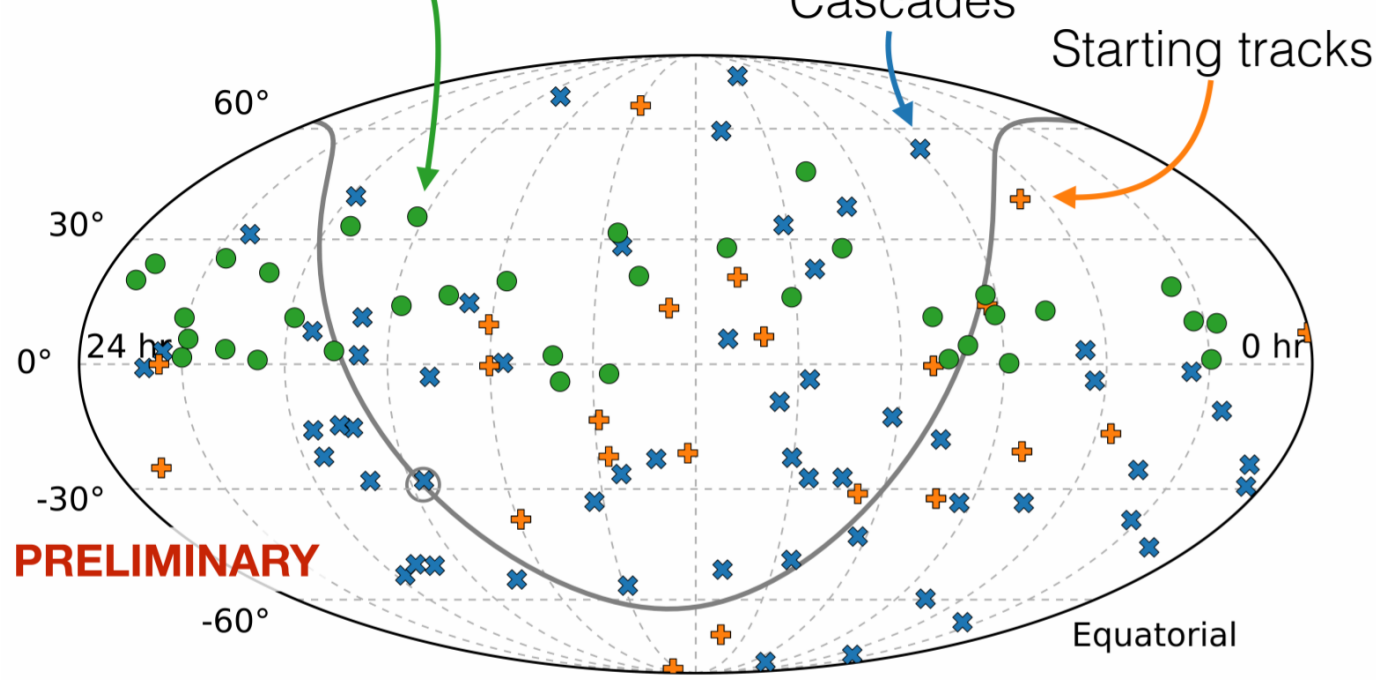

Figure 1.4: Arrival directions of the highest energy IceCube events in equatorial coordinates. Different event morphologies (see Section 1.2.1) are represented by differently colored symbols. The gray line indicates the Galactic plane. Figure reproduced from [19].

\footnotetext{
${ }^{3}$ The IceCube background is dominated by atmospheric muons and neutrinos. See Section 2.1.1 for a more detailed description.
} 

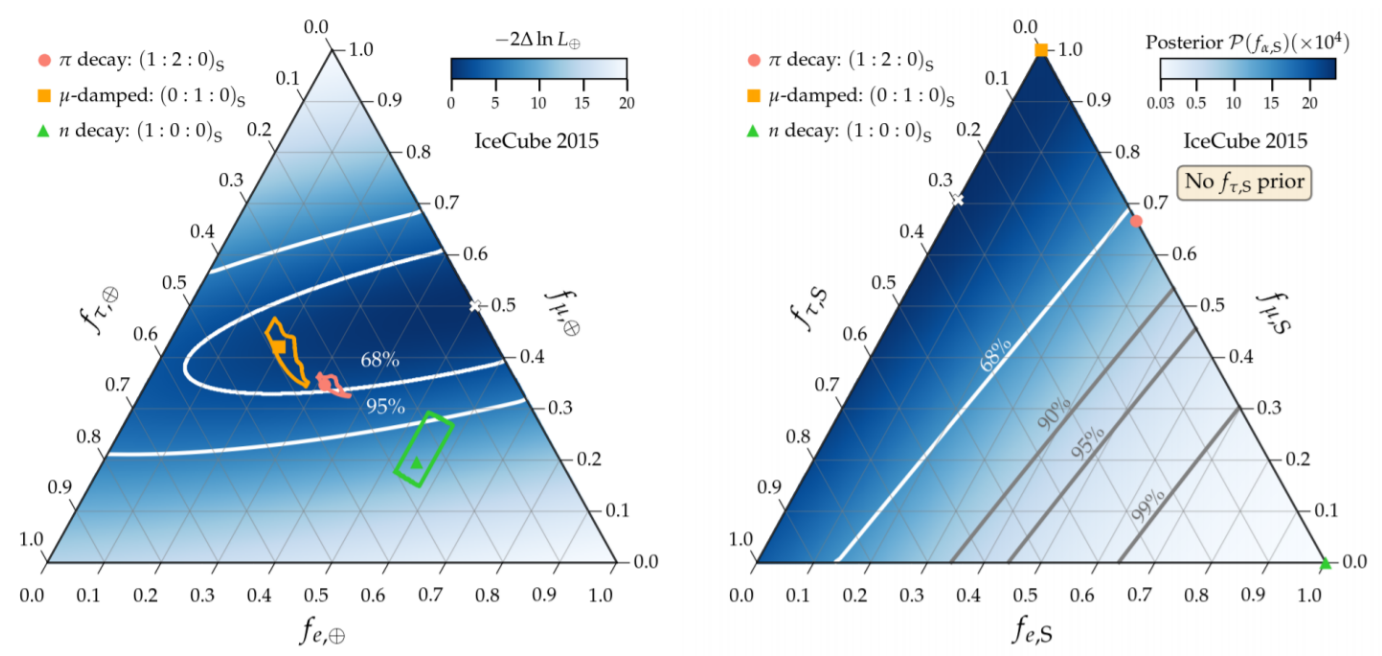

Figure 1.5: Flavor composition of astrophysical neutrinos at the Earth (i.e. measured by IceCube, expressed in terms of likelihood variation $-2 \Delta \ln L$; left) and inferred production ratio at their sources (right). Every point in this "flavor triangle" represents a possible flavor composition $\left(\nu_{e}: \nu_{\mu}: \nu_{\tau}\right)$ (no distinction is made between neutrinos and antineutrinos) at the Earth, " $\oplus$ ", and at the source, "S". Several C.L. contours are labelled in white and gray solid lines. Three benchmark production scenarios (see Section 2.2) and their $3 \sigma$ ranges are shown as red/orange/green points and contours, respectively. The resulting compositions at the Earth are obtained after simulating decoherent neutrino oscillations (see Section 2.3.1). Figure reproduced from [20].

The astrophysical neutrino flux is commonly fitted by a power-law anchored at $100 \mathrm{TeV}$,

$$
\Phi_{\nu}\left(E_{\nu}\right)=\Phi_{\text {astro }} \times 10^{-18}\left(\frac{E_{\nu}}{100 \mathrm{TeV}}\right)^{-\gamma_{\text {astro }}}\left[\mathrm{GeV}^{-1} \mathrm{~cm}^{-2} \mathrm{~s}^{-1} \mathrm{sr}^{-1}\right],
$$

with its overall normalization $\Phi_{\text {astro }}$ and spectral index $\gamma_{\text {astro }}$ as free parameters for the fit. An accurate measurement of these quantities gives us valuable information about the neutrino sources, such as their luminosities, primary proton acceleration mechanisms and viable neutrino production scenarios. Several samplings of the IceCube events have been analyzed, each one preferring a unique combination of $\Phi_{\text {astro }}$ and $\gamma_{\text {astro }}$ (see Section 2.1.2). For reference values, a combined analysis of all astrophysical events by mid-2015 found $\gamma_{\text {astro }}=2.50 \pm 0.09$ and $\Phi_{\text {astro }}=6.7_{-1.2}^{+1.1} \mathrm{GeV}^{-1} \mathrm{~cm}^{-2} \mathrm{~s}^{-1} \mathrm{sr}^{-1} \cdot[21]$ 


\subsection{1}

\section{Event Morphologies}

Each DOM at IceCube contains a photomultiplier tube which indirectly detects neutrinos as they interact with nuclei from the surrounding ice, producing Cherenkov light. From each signal, IceCube can extract the incoming neutrino energy and the direction where it came from, pointing directly back to its source. The precision of such an event reconstruction depends on several factors such as the vertex location, the initial neutrino energy and the nature of the interaction. In this section, we will focus on the latter effect, relating each neutrino interaction to its distinct signature/morphology in the DOM array.

Neutrinos are only subject to weak interactions, meaning they can interact with matter via the exchange of a $Z$ boson (called a neutral current (NC) interaction) or $W^{ \pm}$bosons (called charged current (CC) interactions). These are represented by the following equations

$$
\begin{gathered}
\nu_{\ell}\left(\bar{\nu}_{\ell}\right)+N \longrightarrow \nu_{\ell}\left(\bar{\nu}_{\ell}\right)+N^{\prime} \quad(\mathrm{NC}), \\
\nu_{\ell}\left(\bar{\nu}_{\ell}\right)+N \longrightarrow \ell^{-}\left(\ell^{+}\right)+N^{\prime} \quad(\mathrm{CC}),
\end{gathered}
$$

where the subscript $\ell=e, \mu, \tau$ refers to a specific lepton flavor and $N$ and $N^{\prime}$ are the initial and final hadronic states of the nucleus, respectively. Kinematically, $\gtrsim \mathrm{GeV}$ neutrinos fall into the high-energy regime of deep inelastic scattering ${ }^{4}[22]$, where $N$ and $N^{\prime}$ are necessarily different (the strong recoil of the struck quark fragments the initial nucleon $N$ ).

In the energy range of the astrophysical neutrinos observed by IceCube ( $\gtrsim \mathrm{TeV}), \nu_{e}$ and $\nu_{\tau} \mathrm{CC}$ interactions, as well as NC interactions of all flavors induce localized particle showers. This leads to an approximately spherical light signal centered at the interaction vertex called a "cascade" (or "shower"). Although the cascade morphology has a relatively poor angular resolution of $>8^{\circ}$ (dominated by ice modelling systematics) [23], its good energy resolution of $\sim 15 \%$ [24] makes this channel appropriate for determining the energy dependence of the astrophysical neutrino flux. This precision is possible because the cascade signal can be totally contained inside the detector, allowing for a calorimetric approach: the amount of Cherenkov light collected by the DOMs is proportional to the incoming neutrino energy.

Another process that can produce cascades (and a few other possible signatures) is the resonant $\bar{\nu}_{e}$ elastic scattering with atomic electrons at the

\footnotetext{
${ }^{4}$ Here, the term "inelastic" refers to collisions where the target nucleon is excited and then fragments into partons which hadronize into a different final state. Conversely, "elastic" scattering does not change the nucleon's state.
} 
$W^{-}$pole, also known as the Glashow Resonance [25, 26]. Its cross section at resonance energy $E_{\nu} \approx m_{W}^{2} / 2 m_{e} \approx 6.3 \mathrm{PeV}^{5}$ is $\sim 3$ orders of magnitude larger than that of NC interactions with nucleons at the same energy. Given the amount of $\mathrm{PeV}$ neutrinos detected at IceCube, one would expect several Glashow Resonance events to have occurred. Only recently there has been a sign of them in a single $\sim 6 \mathrm{PeV}$ cascade [27], but this discussion is beyond the scope of this thesis.

The remaining $\nu_{\mu} \mathrm{CC}$ interactions produce muons which can propagate through the ice for several kilometers, resulting in elongated tracks of Cherenkov radiation appropriately called " $\mu$-tracks". Muonic decays of $\tau$ leptons $\left(\tau^{-} \longrightarrow \nu_{\tau}+\mu^{-}+\bar{\nu}_{\mu}\right.$, branching ratio $\left.\sim 17 \%\right)$ produced in $\nu_{\tau} \mathrm{CC}$ interactions also produce tracks as the $\mu$ passes through the detector. Their exceptional angular resolution $\left(<1^{\circ}\right)[28]$ makes them ideal for point-source searches, but their sizeable energy uncertainty (factor of $\approx 2$ ) reflects into large error bars in flux measurements. For illustration, Figure 1.6 shows actual cascade $(1.6(\mathrm{a}))$ and $\mu$-track (1.6(b)) events at IceCube, corresponding to reconstructed neutrino energies $E_{\nu}=1.14 \mathrm{PeV}$ and $E_{\nu}=880 \mathrm{TeV}$, respectively.

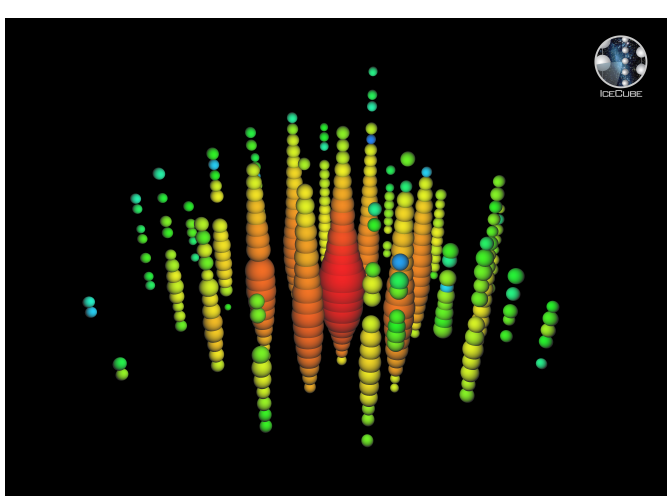

(1.6(a)) Cascade

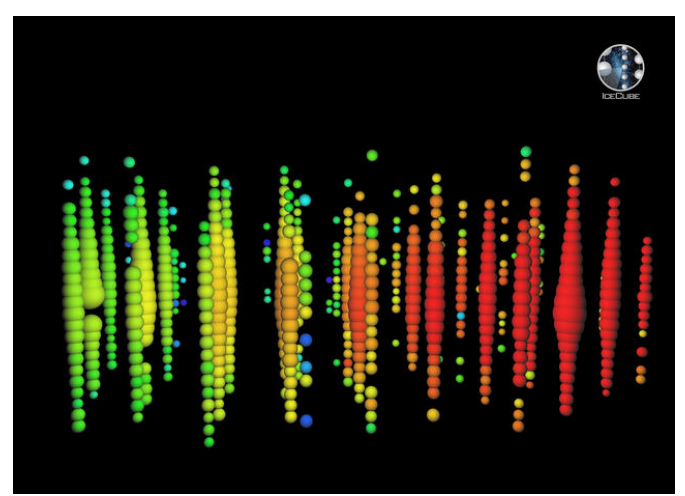

(1.6(b)) $\quad \mu$-track

Figure 1.6: A cascade $^{6}$ (left, $E_{\nu}=1.14 \mathrm{PeV}$ ) and a $\mu$-track (right, $E_{\nu}=880$ $\mathrm{TeV}$ ) at the IceCube detector: two astrophysical neutrino events with distinct morphologies. DOMs are represented by spheres. Their size indicates the amount of Cherenkov light collected by each DOM. Color represents time: in the track signature, the muon travels from right to left. These (and many more) pictures can be found in the official IceCube website [29].

Aside from cascades and $\mu$-tracks, there are other possible event morphologies at IceCube, the most important being the "double cascades" or "double bang" signatures. These are caused by $\nu_{\tau} \mathrm{CC}$ interactions at energies above

\footnotetext{
${ }^{5}$ This energy corresponds to the peak of the cross-section for $\bar{\nu}_{e}+e^{-} \rightarrow W^{-} \rightarrow$ hadrons at center of mass energy $m_{W}^{2}$.

${ }^{6}$ This cascade is called Ernie. Bert (also a cascade) and Ernie were the first two PeV events at IceCube!
} 
$\sim 100 \mathrm{TeV}$. The neutrino-nucleus vertex produces a cascade just like before, but the resulting $\tau$ lepton has enough energy to travel a considerable distance before decaying, $\tau^{-} \longrightarrow \nu_{\tau}+e^{-}+\bar{\nu}_{e}$ or $\tau^{-} \longrightarrow \nu_{\tau}+$ hadrons (branching ratio $\sim 82 \%$ ), producing a second, distinguishable shower. Recently, two double cascade events allowed IceCube to identify its first two $\nu_{\tau}$ candidates (single cascades cannot distinguish $\nu_{e}$ from $\nu_{\tau}$ ) [30]. For a detailed description of other possible $\nu_{\tau}$ signatures at IceCube ("lollipop", "double pulse", "sugardaddy", etc.), see [31]. We conclude this section by summarizing the main morphologies and their corresponding interactions in Table 1.1.

Table 1.1: IceCube's main signatures and their corresponding $\nu$ interactions.

\begin{tabular}{c|c} 
Event Morphology & Interactions \\
\hline Tracks & $\begin{array}{c}\nu_{\mu} \text { CC interactions } \\
\nu_{\tau} \text { CC interactions (only if } \tau \text { decays muonically) } \\
\text { Atmospheric muons (background) }\end{array}$ \\
\hline Single Cascades & $\begin{array}{c}\text { All-flavor NC interactions } \\
\nu_{e} \text { and low-energy } \nu_{\tau} \text { CC interactions }\end{array}$ \\
\hline $\begin{array}{c}\text { Double Cascades } \\
\text { ("double bang") }\end{array}$ & $\bar{\nu}_{e}$ CC interactions with atomic electrons (Glashow Resonance) \\
\hline
\end{tabular}

\section{3}

\section{Searches for Astrophysical Neutrino Sources}

As a natural follow-up from IceCube's observations, physicists and astronomers have been searching for the sources of these astrophysical neutrinos for many years. In this section we will summarize the efforts by the community to tackle this question, which has proven to be quite challenging. At present, several speculated candidates have already been excluded by directional or time correlation analyses, but unfortunately (or fortunately, depending on who you ask) the problem remains open.

There are quite a few known classes of astrophysical objects capable of accelerating particles to high enough energies such that they can produce $\mathrm{TeV}-\mathrm{PeV}$ neutrinos. Among them are:

( $i$ ) Active Galactic Nuclei (AGN): central regions ( $\lesssim 10$ pc radius) of "active" galaxies, where huge amounts of gas accumulate around a supermassive black hole (SMBH), forming an accretion disk surrounded by a larger torus of dust. Astronomers have historically observed a large "zoo" of astrophysical phenomena, now associated to AGN in what is called the "AGN Unification" [32]. They are generally classified by their spectral features/orientation ("Type I": broad spectral lines caused by the AGN's dusty torus standing 
between us and the accretion disk; "Type II": narrow spectral lines when the AGN core is not covered by the torus), radio-wave luminosity (radio-loud vs. radio-quiet, depending on the SMBH's spin and magnetic flux) and nuclear luminosity/accretion rate (i.e. the rate at which the SMBH swallows mass).

Most radio-loud AGN can produce relativistic jets perpendicular to their accretion disk, accelerating particles to near the speed of light. These include blazars and radio galaxies (RGs), which are going to be very important throughout this work. Blazars are AGN whose jets are pointing towards us, and are subdivided into two sub-categories: BL Lacertae objects (or BL Lacs) and flat-spectrum radio quasars, the latter generally more luminous and with stronger emission lines. RGs are similar to blazars, but their jets are obliquely aligned to us, which is why they are sometimes called "misaligned AGN". Ultimately, AGN are expected to accelerate protons up to $\sim \mathrm{EeV}$ energies [33], making them strong candidates for producing the TeV-PeV IceCube neutrinos ${ }^{7}$ $[34,35,36,37]$. Observation of such neutrinos from AGN would prove the existence of hadronic ( $p p$ and $p \gamma)$ interactions at their sources, while their non-observation indicates a mainly leptonic (synchrotron or inverse Compton) origin to AGN $\gamma$-rays. Both scenarios are compatible with multi-wavelength observations, including the ubiquitous double bump feature in blazar spectral energy distributions [38].

(ii) Starburst Galaxies (SBGs): galaxies undergoing a period of exceptionally high star-formation rate (SFR), converting gas into stars $\left(10-100 \mathrm{M}_{\odot} /\right.$ year, as compared to the Milky Way's $\sim 3 \mathrm{M}_{\odot} /$ year $)$. This is usually triggered by galaxy mergers, fuelling the central bulge of the merger remnant with large gas densities [39]. SBGs contain high velocity stellar winds and host several supernovae which can accelerate protons up to $E_{p} \sim 100 \mathrm{TeV}^{8}$, producing neutrinos in $p p$ interactions (See Section 2.2.1) within the IceCube range $[41,42,43,44]$.

(iii) Gamma-Ray Bursts (GRBs): the most energetic explosions observed in the Universe, believed to result from core-collapse supernovae, producing long-lasting GRBs of $>2 \mathrm{~s}$, or from compact object mergers such as two neutron stars or a neutron star and a black hole, producing short GRBs of $\sim 0.2 \mathrm{~s}$ on average. These transient events can outshine their entire host galaxies (usually ones with a high SFR such as SBGs), converting most of their energy

\footnotetext{
${ }^{7}$ Although jetted AGN are way more popular PeV neutrino candidates, non-jetted AGN can also account for a fraction of the lower-energy IceCube events.

${ }^{8} \mathrm{~A}$ smaller fraction of more powerful hypernovae could potentially reach $E_{p} \sim 100 \mathrm{PeV}$
} [40]. 
output (up to $\sim 10^{51} \mathrm{erg}$ ) into $\gamma$-rays collimated in oppositely directed jets. An afterglow of gradually increasing wavelengths (X-ray, ultraviolet, optical, infrared, microwave and finally radio) can be seen for several days following the initial $\gamma$-ray signal. GRB environments are transparent to GWs and neutrinos, making them viable candidates for explaining the IceCube observations $[45,46]$. These neutrinos are dominantly produced around $E_{\nu} \sim 100 \mathrm{TeV}$ but may reach $\sim \mathrm{EeV}$ energies in extreme cases of massive star collapse [47].

(iv) Supernova Remnants (SNRs): structures resulting from supernova explosions, most of them spotted within our own galaxy. Their shocks are likely able to accelerate protons up to $\mathrm{PeV}$ energies [48], possibly accounting for a fraction of sub-100 TeV neutrino events at IceCube [49, 50, 51, 52]. Supernovae can also form highly magnetized neutron stars called pulsars, located at the center of SNRs. Pulsar winds can power nebulae (clouds of dust and ionized gases) inside SNRs, creating pulsar wind nebulae (PWN) which are the dominant $\gamma$-ray emitters in our Galaxy and possible contributors to the IceCube neutrino flux.

(v) Tidal Disruption Events (TDEs): stars being captured by a SMBH's strong tidal force. This destructive event produces intense flares of electromagnetic radiation as the star's mass gets captured into the accretion disk. Jetted TDEs can accelerate protons up to $E_{p} \sim 10^{20} \mathrm{eV}$, producing neutrinos in photohadronic interactions [53]. These jets can also be "choked", suppressing their $\gamma$-ray emission while keeping the neutrino flux intact. Numerical simulations have shown that TDEs can account for at least $\sim 10 \%$ of the IceCube flux at $\sim 1 \mathrm{PeV}[54]$.

$\gamma$-ray experiments have catalogued over $10^{5}$ point-like objects belonging to these and other candidate classes. Thousands of them have been selected to undergo IceCube's neutrino point-source searches, adopting different strategies depending on the type of source being considered. However, all of them require a sample of IceCube events with high statistics and good angular resolution. As we have already seen, $\mu$-tracks provide exactly that, although some analyses have been carried out using cascade events and, therefore, all three neutrino flavors [23, 55].

Time-integrated searches pertain to "permanent" objects ${ }^{9}$ such as AGN, SBGs, SNRs and PWN, looking for coincidences in both direction and energy of neutrino events with possible sources. The idea is to divide the sky into

${ }^{9}$ Of course, they are technically not permanent, but can survive for millions of years. 
a fine grid and look for clusterings of events in each directional bin which are incompatible with the background-only hypothesis. Lack of significant clustering when comparing with several catalogs $[56,57,58]$ has resulted in null searches so far $[59,60,61,62,63,64]$. Null results are not useless: they allow us to establish upper limits on the $\nu_{\mu}+\bar{\nu}_{\mu}$ flux coming from these sources. They can also exclude (or at the very least challenge) certain models for neutrino production at specific source classes.

These time-integrated analyses also consider the possibility that multiple individual sources may be too faint to signal any coincidence with neutrino events. Instead, they may be revealed collectively once they are summed up (i.e. stacked together) by using a stacking technique described in [64] and [65]. Additionally, extended regions of neutrino emission (as opposed to point sources) such as large galaxy clusters, the "Fermi Bubbles" [66] or diffuse galactic emissions coming from or unresolved SNRs have also been considered $[61,67]$.

It is worthwhile mentioning that $\mathrm{a} \sim 3 \sigma$ coincidence between a $\sim 290 \mathrm{TeV}$ track labelled IceCube-170922A and the flaring blazar ${ }^{10}$ TXS 0506+056 has been found in an extensive multi-wavelength campaign by several collaborations [68]. Complementary results also reveal evidence of multiple neutrino events in the same direction prior to the flaring episode [69]. Although this might indicate a blazar origin to the astrophysical neutrinos, other studies have shown that they can only account for a small fraction of IceCube's diffuse neutrino flux [70].

For transient sources such as flaring blazars, TDEs or GRBs, one requires an additional condition: the neutrino and $\gamma$-ray signals must be correlated not only in direction but also in time (typically, a \pm 2 -hour window between both events is required). To achieve this, IceCube adopts an unbinned maximumlikelihood method described in detail in [71] and [72]. Needless to say, these searches have so far only set constraints on GRB models and their capacity to accelerate protons to produce ultra-high energy $(>\mathrm{EeV}$ ) cosmic rays (UHECRs) $[73,74,75,76]$.

Aside from $\gamma$-ray multimessenger searches, UHECRs have also been used to look for neutrino point-sources [77]. This analysis is considerably more challenging because magnetic field deflections can erase any directional information from the CR signal, as well as induce significant delays between the neutrino and CR arrival times. On the other hand, CRs can be used in different ways to extract information about IceCube neutrino sources. For instance, an

\footnotetext{
${ }^{10}$ Blazars can go from their quiescent/regular state to a flaring state, increasing by many orders of magnitude their jet emission of optical $/ \gamma$-rays. Determining the cause and frequency of these flares is still an active field of research.
} 
important result was obtained in [78], where a model-independent upper limit to the intensity of the astrophysical neutrino flux,

$$
E_{\nu}^{2} \Phi_{\nu}\left(E_{\nu}\right) \lesssim 2 \times 10^{-8} \mathrm{GeVcm}^{-2} \mathrm{~s}^{-1} \mathrm{sr}^{-1} \quad \text { for } E_{\nu} \in\left[10^{14}, 10^{16}\right] \mathrm{eV}
$$

was set based on the observed flux of UHECRs. This is known as the WaxmanBahcall bound (although it is sometimes treated as a ballpark value, since the assumptions behind it are not necessarily true), which is so far consistent with the IceCube observations.

\section{4}

\section{Objectives}

Now that we have presented a brief overview of multimessenger astrophysics, focusing on the status of neutrinos as cosmic messengers, we are ready to lay out the objectives of this dissertation. Our main goal is to devise and implement a multimessenger method for establishing quantitative bounds on the sources of the IceCube neutrino flux. In order to do that, we will follow a series of steps:

1. Explain in greater detail the IceCube measurements, including their backgrounds, datasets and flux fits;

2. Assuming standard neutrino production scenarios, understand the connection between the neutrino and $\gamma$-ray emissions at astrophysical sources;

3. Study the propagation of these particles through intergalactic space, especially the electromagnetic cascade induced by $\gamma$-ray interactions with cosmic photon backgrounds;

4. Introduce a parametrization for neutrino injection spectra consistent with the IceCube observations and calculate the corresponding $\gamma$-ray spectra observed at the Earth;

5. Develop a statistical method for testing the compatibility between neutrino and $\gamma$-ray data, providing physical interpretations for our results.

The results presented in this work have been published in [79] and complemented in [80]. Here, we will focus on further describing the theoretical background, analysis methodology and results obtained in [79], without extending our discussion to the latter paper. 
A secondary goal of this thesis is to assist other fellow physicists who might be interested in entering the field of multimessenger astrophysics. For this reason, some references have been included to direct the reader towards useful reviews on certain subjects. Although a previous knowledge of cosmology is assumed, Appendix A conveniently summarizes everything one should know, starting from the Friedmann-Lemaître-Robertson-Walker (FLRW) metric.

With all our intentions made clear, let us begin! 


\section{2 \\ Multimessenger Astrophysics}

In this chapter we describe in detail the realm of multimessenger astrophysics (specifically regarding neutrinos and $\gamma$-rays) which is the main topic of this dissertation. A previous knowledge about general relativity and cosmology will be assumed; concepts like comoving distance, Hubble parameter and cosmological redshift are used in this chapter without being introduced to the reader. A useful (and relatively basic) review on the subject can be found in Appendix A. We will also make extensive use of the terminology common in the fields of astronomy and astrophysics.

We will start this chapter by presenting three IceCube datasets which will be considered in our analyses. It is important to comprehend the characteristics of the neutrino flux reported in each dataset (their fits to powerlaw spectra with specific normalizations and spectral indices, as well as their threshold energies based on IceCube's background rejection methods) in order to understand the main results of this thesis.

Next, we will briefly review the conventional production mechanisms of high energy particles in astrophysical sources, emphasizing the connection between the emission of neutrinos and $\gamma$-rays. This is a central piece to this work as it justifies our multimessenger approach. Finally, we will talk about neutrino propagation and establish a simple relation between the neutrino emission spectrum and the neutrino flux (measured by IceCube) at the Earth. On the other hand, the propagation of $\gamma$-rays is not as straightforward and, due to its complexity and technicality, deserves a chapter of its own. 


\section{1}

\section{Event Selection at IceCube}

\subsection{1}

\section{Atmospheric Backgrounds}

Although we will be considering in this work the astrophysical neutrino flux measured by IceCube, it is important to note that most of the events registered by the PMTs in the detector actually come from atmospheric backgrounds. CRs interact with gas in the Earth's atmosphere, producing muons and neutrinos in air showers with energies up to $\sim 100 \mathrm{TeV}$. This is well within the range of energies that IceCube has reported observation of astrophysical neutrinos, which means that such backgrounds must be well understood in order to distinguish them from actual signals of extraterrestrial neutrinos.

Atmospheric muons dominate IceCube's background in the search for astrophysical neutrinos, with a rate of $3000 \mu$-tracks per second. One way of eliminating these events is to consider only upgoing tracks since muons cannot traverse the Earth to reach the detector, at the cost of missing half of the sky (including the Galactic center). Another way is to impose a high-energy cut $(\gtrsim 1 \mathrm{PeV})$ in the event selection [81], making the detector sensitive only to very high energy neutrinos. Finally, one can filter out most of the entering tracks by considering only events where the interaction vertex is inside the detector.

Aside from muons, neutrinos are also produced in air showers from CR interactions in the atmosphere, which IceCube should be able to discern from astrophysical neutrinos. Before its completion, IceCube's 59-string configuration was seeing $\approx 80$ atmospheric neutrino events per day after thorough event selection and classification [82]. Most of the contribution to this atmospheric neutrino background comes from the decay of pions and kaons, known as the conventional atmospheric neutrino flux, which extends up to $E_{\nu} \approx 500 \mathrm{TeV}$. Its typical spectral behaviour follows an $E_{\nu}^{-3.7}$ power law, which is softer than the $\mathrm{CR}$ spectrum of $E^{-2.7}$ because these light mesons lose energy before decaying (see [83] for a complete derivation of the spectral relations between CRs and atmospheric neutrinos). ${ }^{1}$ Another source of neutrinos which dominates at $E_{\nu} \gtrsim 100 \mathrm{TeV}$ arises from the decay of heavier, charmed mesons (e.g. $D^{ \pm}$), which have short lifetimes. This causes them to decay promptly (thus the name "prompt atmospheric neutrino flux"), without re-interacting, such that

\footnotetext{
${ }^{1}$ This spectrum actually varies depending on the zenith angle. This is because horizontally travelling mesons have a higher probability of decaying before losing energy in collisions. Hence, the conventional atmospheric neutrino flux is harder for horizontal directions and softer in the vertical direction.
} 
they follow the CR $E_{\nu}^{-2.7}$ spectrum up to $500 \mathrm{TeV}$. This prompt atmospheric neutrino flux component has not yet been observed by IceCube.

While muons are only able to reach the detector through the southern sky, neutrinos produced in the northern hemisphere are able to travel through the Earth and can be detected by IceCube. A simple directional restriction cannot eliminate such background. Instead, one must use the fact that such neutrinos are often accompanied by penetrating muons (in reactions such as $\pi^{+} \longrightarrow \mu^{+}+\nu_{\mu}$ for example), which is never the case for astrophysical neutrinos since these muons are lost over cosmological distances. This so-called "selfveto" method was first proposed in [84] (and later expanded in [85]), where they showed that atmospheric neutrinos with $E_{\nu}>10 \mathrm{TeV}$ coming from a zenith angle $<60^{\circ}$ has a $99 \%$ chance of being vetoed. Such a technique plays a crucial role for IceCube to select its signal events amidst so much noise coming from the atmosphere.

\subsection{2}

\section{IceCube Datasets}

We will be considering three datasets in this work which IceCube uses to classify its events. They are separated based on different background rejection methods, which we will describe soon. They are the following:

(i) High Energy Starting Events (HESE): any event (track or shower) where the origin of the signal (i.e. the interaction vertex) is contained within the fiducial volume of the detector;

(ii) Cascade events: events with cascade-like morphology (see Introduction for the different event morphologies);

(iii) Through-going $\mu$-tracks: Cherenkov tracks coming from the northern hemisphere, relaxing the requirement that events begin inside the detector.

This section serves to introduce these datasets, describing their main backgrounds and how they are dealt with, as well as showing how they have changed over the years. 


\subsubsection{1 HESE Dataset}

To tag the events that make it into the HESE dataset, a veto region is defined in the outer layer of the instrumented volume, where any event producing first light in this region is discarded as an entering track. This greatly reduces the background as only events that started within the fiducial volume (i.e. not in the veto region) or had high enough energies that the corresponding $\mu$-track would have been identified reliably were selected.

This veto region is also used to filter out most of the atmospheric neutrino background via the previously mentioned self-veto technique. Recall that such neutrinos are normally produced together with a muon which can be detected in the veto region almost simultaneously to the neutrino event. Thus, by discarding any signal around a 50 ns window of a muon detection in the veto region, IceCube is able to identify whenever a neutrino was atmospheric or astrophysical with a high precision. The efficiency decreases as the neutrino energy gets smaller because the angular separation between the $\nu$ and $\mu$ can get large enough such that only the neutrino passes through the detector. A first analysis by the IceCube Collaboration [15] has determined that events with energy $E_{\nu}<E_{t h}=60 \mathrm{TeV}$ should be excluded from the HESE dataset in order to avoid a large background contamination. However, IceCube also has a low-energy extension of the HESE dataset called the Medium Energy Starting Events (MESE) [86], lowering the threshold down to $25 \mathrm{TeV}$.

The HESE dataset has been updated several times since its first announcement in [15], which itself was a follow-up on the first observation of two PeV-energy neutrinos at IceCube [14]. Figure 2.1 shows the number of HESE events for each sample update in the past. The current HESE data is displayed in Figure 2.2, with a total of 7.5yr collecting events.

Recall that the astrophysical neutrino flux is fitted by a single power-law of the form

$$
\Phi_{\nu}\left(E_{\nu}\right)=\Phi_{\text {astro }} \times 10^{-18}\left(\frac{E_{\nu}}{100 \mathrm{TeV}}\right)^{-\gamma_{\text {astro }}} \quad, \text { for } E_{\nu} \geq E_{\mathrm{th}} .
$$

The fit to the most recent HESE 7.5yr dataset results in an all-flavor normalization of $\Phi_{\text {astro }}=6.45_{-0.46}^{+1.46}\left[\mathrm{GeV} \mathrm{cm}{ }^{-2} \mathrm{~s}^{-1} \mathrm{sr}^{-1}\right]$ and a spectral index of $\gamma_{\text {astro }}=2.89_{-0.19}^{+0.2}$, with errors within a $68.3 \%$ confidence interval. These values, together with the threshold energy $E_{\mathrm{th}}=60 \mathrm{TeV}$, below which the data has not been considered in the fit, will be important to our analysis. 


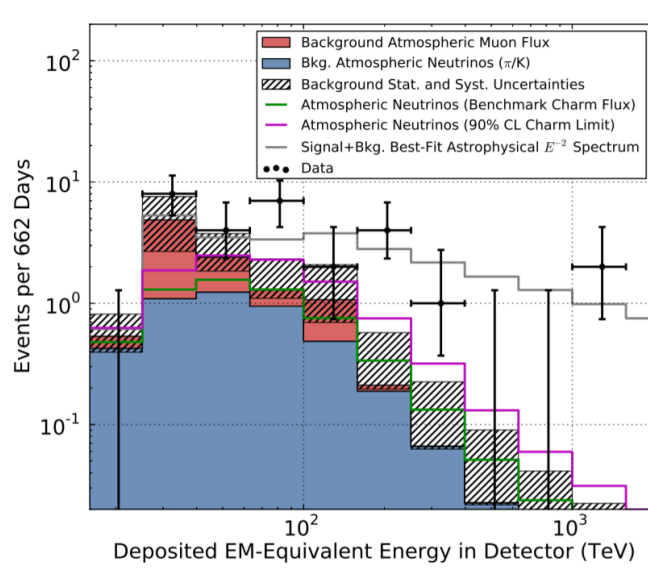

(2.1(a)) HESE 2yr

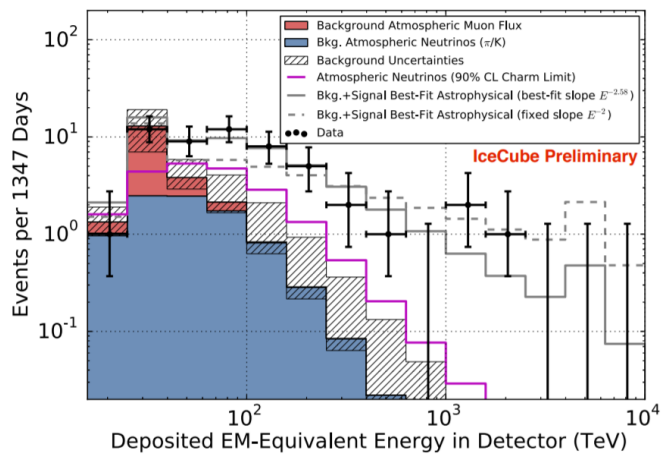

(2.1(c)) HESE 4yr

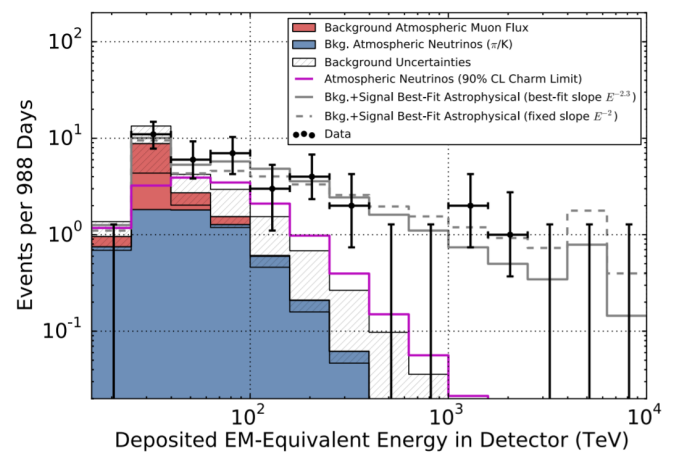

(2.1(b)) HESE 3yr

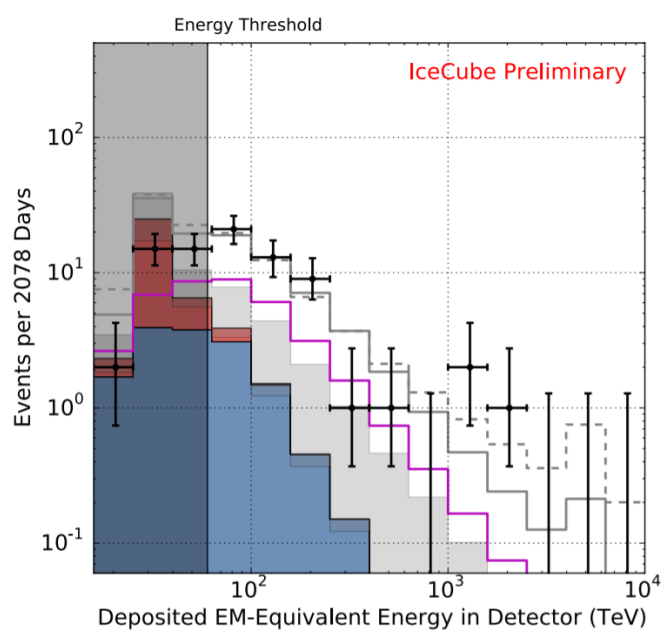

(2.1(d)) HESE 6yr

Figure 2.1: Number of events per deposited energy after 2 years [15] (2.1(a)), 3 years [18] (2.1(b)), 4 years [87] $(2.1(\mathrm{c}))$ and 6 years [88] (2.1(d)) of HESE data. Expected backgrounds due to atmospheric muons and neutrinos, as well as their uncertainties are indicated in the red, blue and hatched regions. Error bars in the data points represent a $1 \sigma$ uncertainty. A total of 28 astrophysical neutrino events were reported in the 2-year sample: an excess with respect to the expected background events at $4.1 \sigma$ confidence level (C.L.). This was followed by 37 candidate events in the 3 -year sample, 54 events after 4 years and 82 in the 6-year HESE sample. The gray shaded region to the left of the vertical line in the 6-year plot marks where $E_{\nu}<60 \mathrm{TeV}$, which has been excluded from this and all previous analyses (see text). 


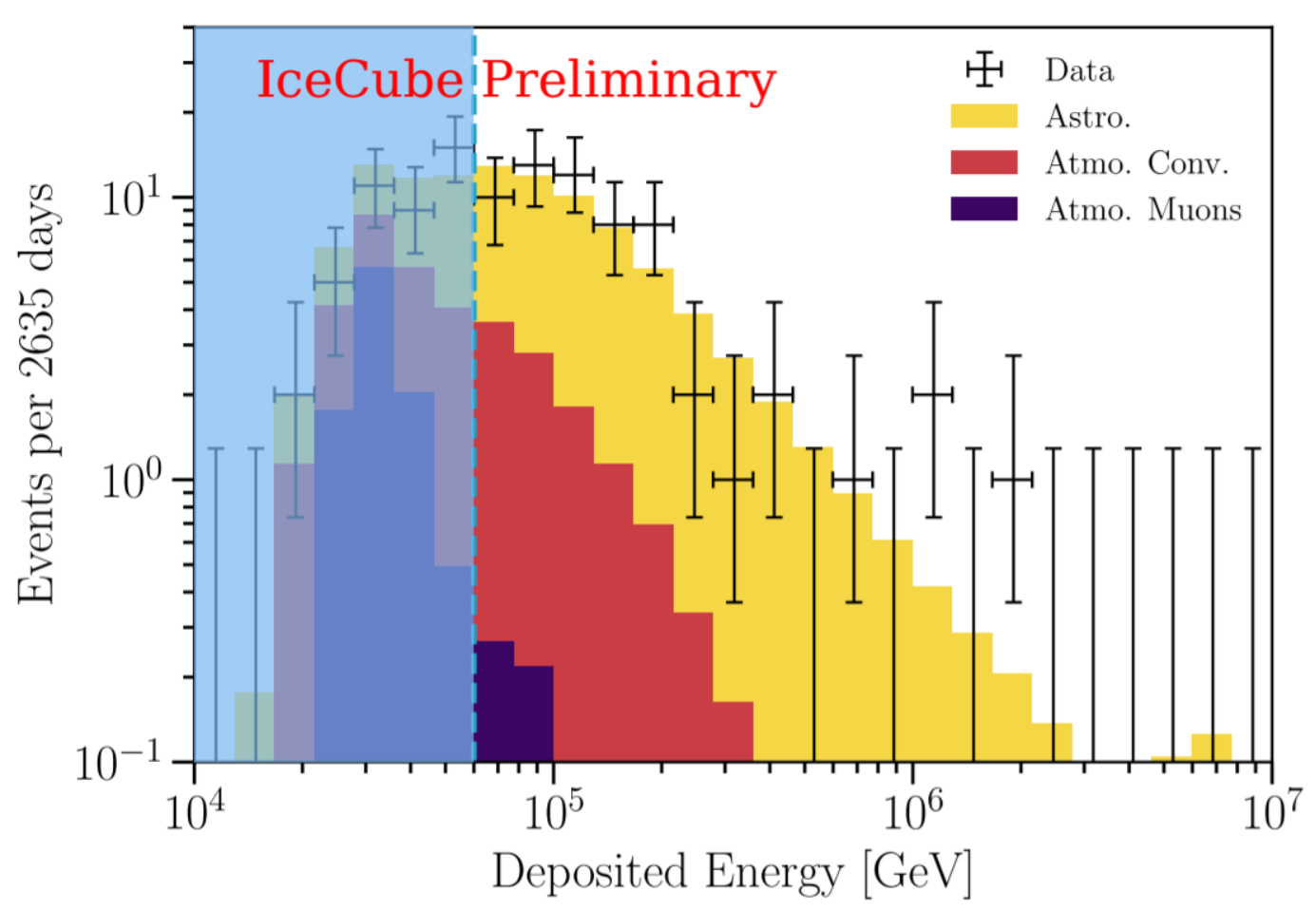

Figure 2.2: Most recent results for deposited energies in HESE, after 7.5 years of data [89]. Data points are black crosses with $1 \sigma$ error bars, and the colored stacked histogram shows the best fit expectations for each component of the total neutrino flux: astrophysical (yellow), conventional atmospheric neutrinos (red) and atmospheric muons (blue). Just as before, events below $60 \mathrm{TeV}$ (shaded region) are excluded from the analysis/fit, leaving us with 60 events above this threshold.

\subsubsection{2}

\section{Cascade Dataset}

Although cascades have a poor angular resolution of $\sim 15^{\circ}$, their good energy resolution $(\sim 15 \%)$ and low atmospheric background turn them into promising candidates to study the astrophysical neutrino flux as a function of energy. The main background in the search for cascades comes from atmospheric muons which produce a faint track and deposit most of their energy in a single bremsstrahlung, mimicking a cascade-like event. A complex filter adopted by IceCube reduces this background from $\sim 2.7 \mathrm{kHz}$ to $\sim 30$ $\mathrm{Hz}$, while keeping $\sim 90 \%$ of the astrophysical neutrinos and $\sim 70 \%$ of the conventional atmospheric neutrinos. Once again, IceCube relies on the selfveto technique to filter out most of this leftover neutrino background.

Figure 2.3 shows the single power-law best-fit astrophysical neutrino fluxes for the 2-year and 4-year cascade samples, while Figure 2.4 presents the current 6-year cascade data, which fits a power-law of the form 2-1 with per-flavor normalization $\Phi_{\text {astro }}=1.66_{-0.27}^{+0.25}\left[\mathrm{GeV} \mathrm{cm} \mathrm{cm}^{-2} \mathrm{~s}^{-1} \mathrm{sr}^{-1}\right]$ and index 
$\gamma_{\text {astro }}=2.53 \pm 0.07$. Ultimately, the sensitive range for this analysis (i.e. the smallest energy range where a non-zero astrophysical component is consistent with the data at $90 \%$ C.L.) is between $16 \mathrm{TeV}<E_{\nu}<2.6 \mathrm{PeV}$, which means that $E_{t h}=16 \mathrm{TeV}$ for the 6yr cascade dataset.

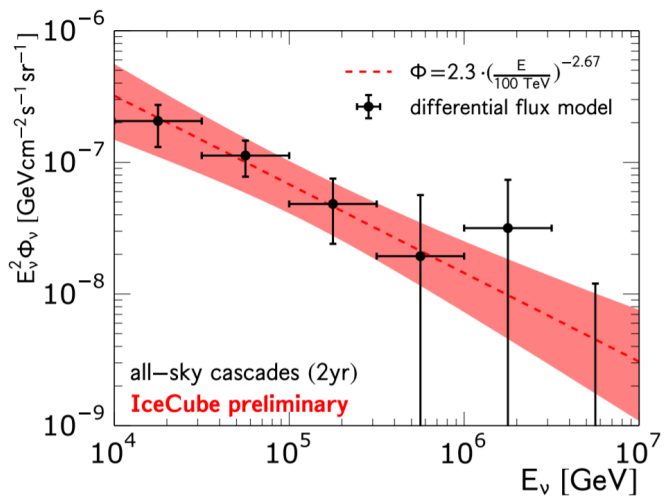

(2.3(a)) Cascade 2yr

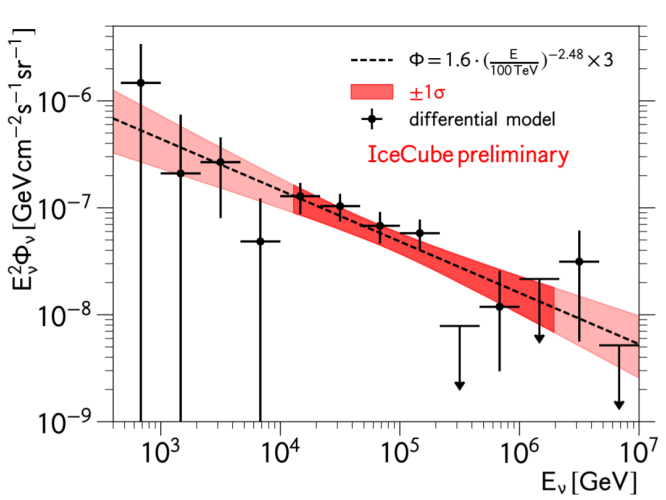

(2.3(b)) Cascade 4yr

Figure 2.3: Measured astrophysical neutrino flux with $1 \sigma$ error bars for IceCube's cascade dataset after 2 years [90] (2.3(a)) and 4 years [91] (2.3(b)) of data-taking. These data points were obtained using a differential model, where an $E^{-2}$ weight was assumed in each energy bin. Best-fit power-laws are represented by dashed lines with a $\pm 1 \sigma$ red band around them. In Figure 2.3(b), the dark red shaded region represents the sensitive energy range (i.e. the energy range included in the power-law fit).

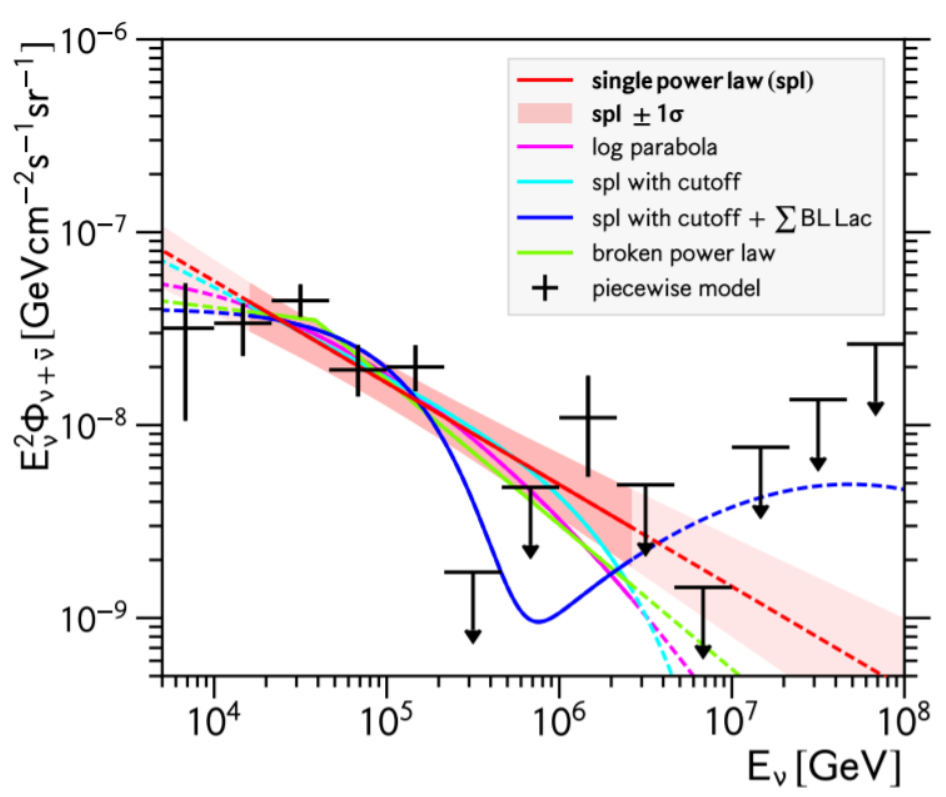

Figure 2.4: Astrophysical neutrino flux with 6 years of cascade events [92]. Crosses represent the differential model best fit results (see caption in Fig. 2.3) and solid (dashed) colored lines represent several flux models in (outside of) the sensitive energy range of $16 \mathrm{TeV}<E_{\nu}<2.6 \mathrm{PeV}$. The red line is the one we are interested in, with a single power-law fit and a $1 \sigma$ red error band around it. 


\subsubsection{3}

\section{Through-going $\mu$-track Dataset}

This last dataset which we shall introduce contains track-like events from neutrinos coming from the northern sky, where the atmospheric muon background is suppressed. In fact, due to the good angular resolution of tracks ( $\lesssim 1^{\circ}$ for $E_{\nu}>1 \mathrm{TeV}$ ), IceCube is able to reject $99.7 \%$ of the muon background at zenith angles between $85-180^{\circ}$, leaving behind only $\mu$-tracks that have been misinterpreted as up-going. At these high energies, the Earth also becomes opaque to neutrinos, causing the signal to be dominated by events near the horizon.

Since 2009, IceCube has presented 5 updates on the search for astrophysical neutrinos using through-going $\mu$-tracks: 1-year [16], 2-year [93], 6-year [94], 8-year [17] and 9.5-year [95] datasets. Figure 2.5 shows the astrophysical neutrino spectrum for the through-going dataset after 6 and 8 years, while Figure 2.6 presents the most recent results. The range of neutrino energies supporting an astrophysical component over a purely atmospheric hypothesis at $90 \%$ likelihood ratio is $40 \mathrm{TeV}-3.5 \mathrm{PeV}$, giving us a theshold energy of $E_{t h}=40$ $\mathrm{TeV}$ for this dataset.

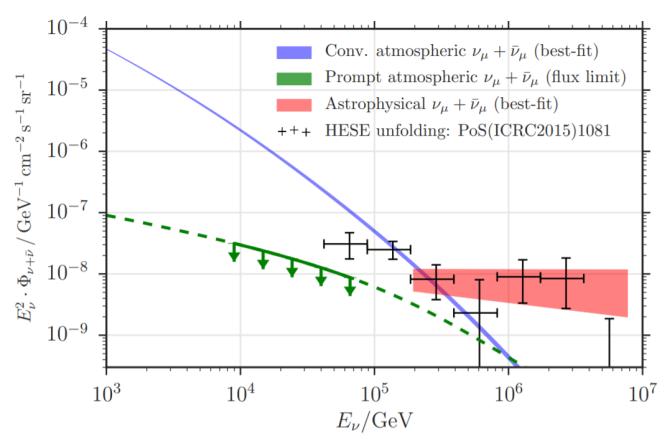

(2.5(a)) Through-going $6 y \mathrm{r}$

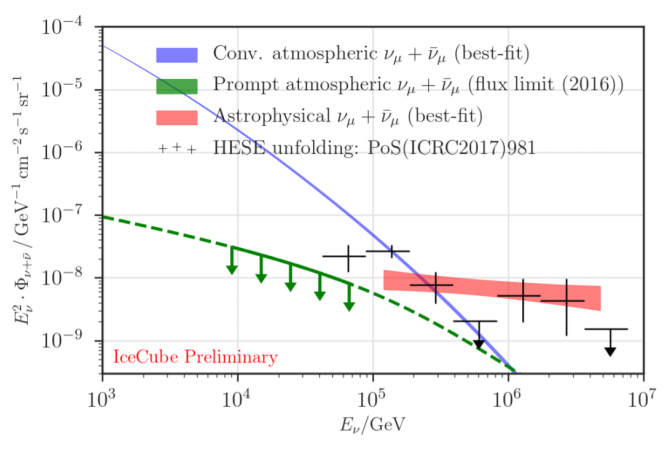

(2.5(b)) Through-going 8yr

Figure 2.5: Best-fit neutrino spectra considering through-going $\mu$-tracks coming from the northern hemisphere after 6 years [94] (2.5(a)) and 8 years [17] (2.5(b)) of collecting data. Expected conventional and (upper bounds for) prompt neutrino contributions are shown in blue and green, respectively.

For this last sample, the best-fit parameters for a single power-law fit of the form 2-1 are $\Phi_{\text {astro }}=1.44_{-0.24}^{+0.25}\left[\mathrm{GeV} \mathrm{cm} \mathrm{cm}^{-1} \mathrm{sr}^{-1}\right]$ (per-flavor) and $\gamma_{\text {astro }}=2.28_{-0.09}^{+0.08}$. A summary of these results for all 3 datasets discussed in this dissertation can be found in Table 2.1 and Figure 2.7 [92]. Although this may seem strange at first since we expect a common origin for the astrophysical neutrinos independently of the morphology of their signals in the detector, these datasets are actually consistent with each other at a $\sim 2 \sigma$ level. 


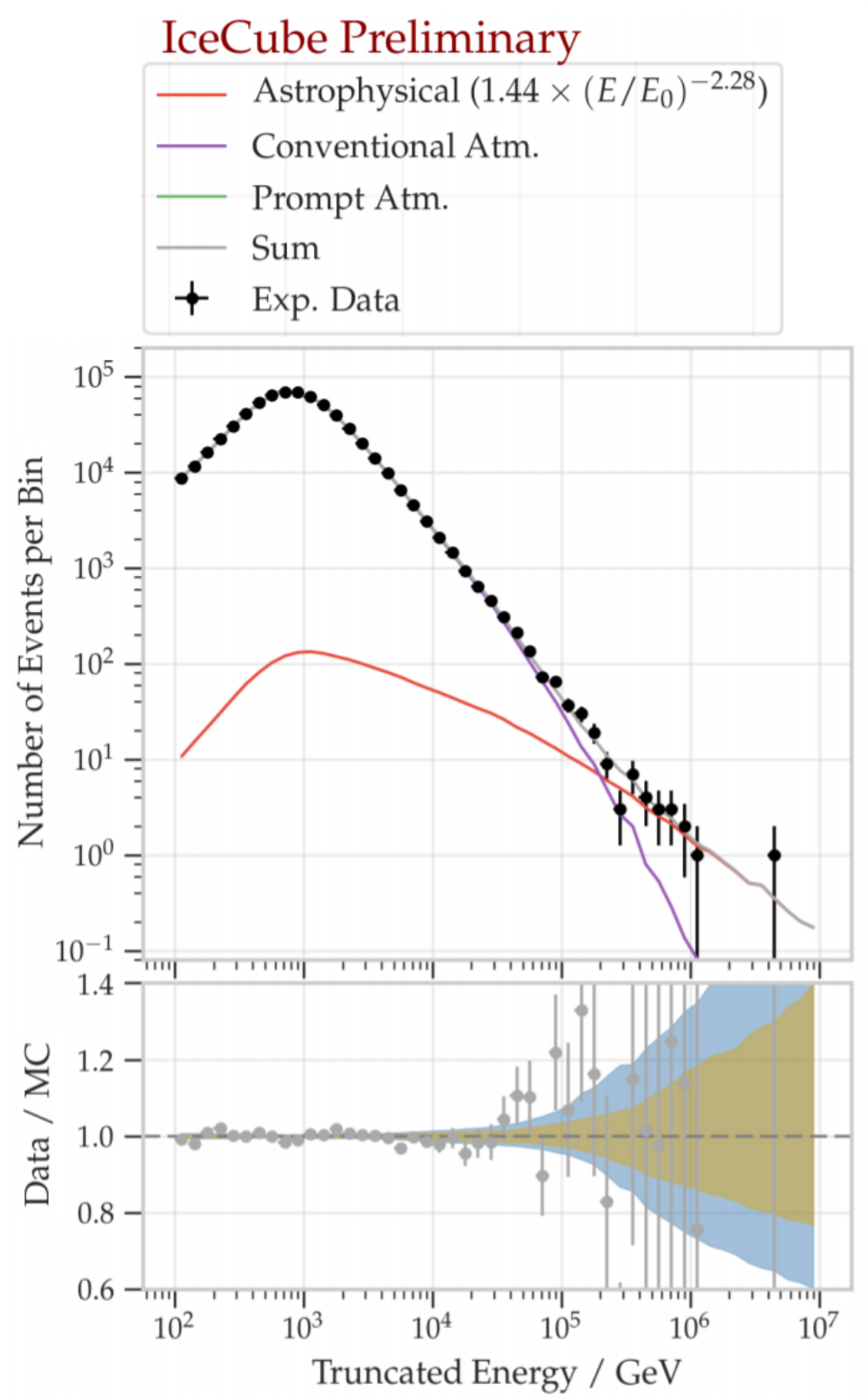

Figure 2.6: Best-fit neutrino spectra with 9.5 years of through-going $\mu$-track data [95]. The sum of the astrophysical (red line) and conventional (purple line) components provide a fit to the experimental data. The prompt atmospheric component (green line) is not shown because its best-fit normalization is zero.

Table 2.1: Best-fit parameters and low-energy threshold energies for each IceCube dataset considered in this thesis. The $\Phi_{\text {astro }}$ reported values are perflavor. In order to obtain each dataset's $\Phi_{\text {astro }}$, IceCube assumes a $(1: 1: 1)$ flavor ratio at the Earth and uses this to extrapolate the normalization of all astrophysical neutrinos from the events of an individual dataset.

\begin{tabular}{c|c|c|c} 
& $\Phi_{\text {astro }}\left[\mathrm{GeV} \mathrm{cm} \mathrm{cm}^{-2} \mathrm{~s}^{-1} \mathrm{sr}^{-1}\right]$ & $\gamma_{\text {astro }}$ & $E_{\text {th }}[\mathrm{TeV}]$ \\
\hline HESE & $2.15_{-0.15}^{+0.49}$ & $2.89_{-0.19}^{+0.2}$ & 60 \\
\hline Cascades & $1.66_{-0.27}^{+0.25}$ & $2.53 \pm 0.07$ & 16 \\
\hline TG & $1.44_{-0.24}^{+0.25}$ & $2.28_{-0.09}^{+0.08}$ & 40
\end{tabular}




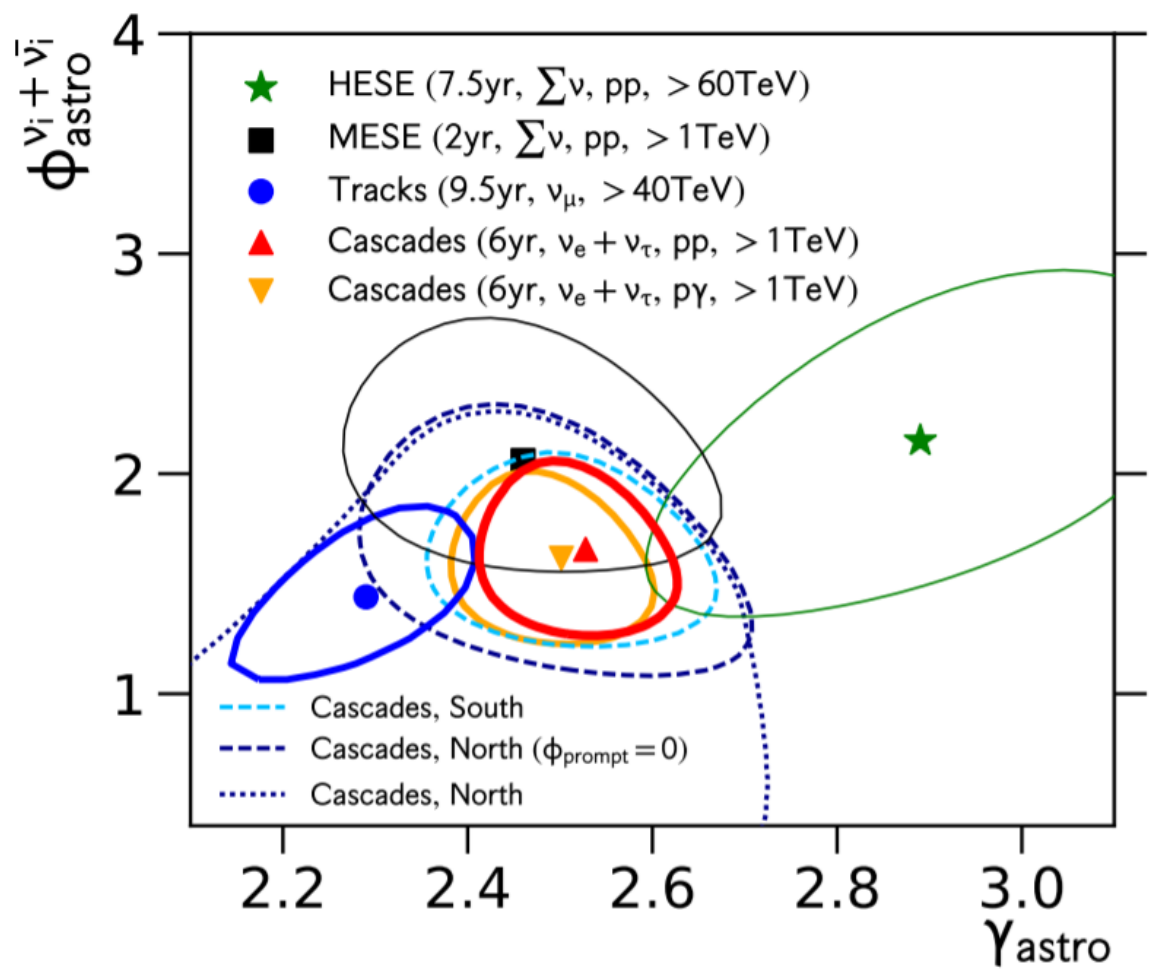

Figure 2.7: Single power-law astrophysical neutrino flux parameters for each IceCube dataset, including their best-fit values and $1 \sigma$ contour around them. The contours which are of interest to us are the HESE (green), Tracks (blue) and Cascades assuming $p \gamma$ production mechanism (yellow). ${ }^{2}$ See Section 2.2 for further details on the production mechanisms. Figure reproduced from [92].

\section{2}

\section{Emission of High-Energy Particles in Astrophysical Sources}

Various astrophysical objects such as AGN and GRBs are sites to look for accelerating charged particles, such as protons and electrons, to extremely high non-thermal energies. Accelerated protons can interact with background gas or radiation present in these dense regions, resulting in the production of high energy neutrinos. There are the two conventional scenarios for how these astrophysical neutrinos are formed: $(i)$ hadronuclear interactions between two protons ( $p p$ scenario) and $(i i)$ photohadronic interactions of accelerated protons with background photons ( $p \gamma$ scenario). In what follows, we shall describe in detail each of these production mechanisms. A useful review on the types of astrophysical sources where these processes may occur can be found in [96].

\footnotetext{
${ }^{2}$ Different production mechanisms lead to different flavor ratios at the Earth (in particular, neutrinos versus antineutrinos), resulting in slightly different fits in each case.
} 


\subsection{1}

\section{pp Scenario}

This scenario is expected to happen in CR reservoirs (e.g. starburst galaxies, galaxy clusters/groups), where CRs that have escaped from their accelerators stay confined by strong magnetic fields. Inelastic $p p$ scattering occurs, leading to the production of charged and neutral pions

$$
p+p \longrightarrow X+\pi^{ \pm, 0}
$$

where $X$ stands for other hadrons. Dominant channels have $X=p+p$ producing $\pi^{0}$ and $X=p+n$ producing $\pi^{+}$; production of $\pi^{-}$becomes more relevant at higher energies. Due to isospin symmetry, these pions are produced with similar probabilities in this process at high energies, $\left(\pi^{+}: \pi^{-}: \pi^{0}\right) \approx(1$ : $1: 1)$. They receive equal amounts $\left(\approx \frac{1}{3}\right)$ of the initial proton's energy, which is later distributed to its decay products, including neutrinos

$$
\begin{aligned}
& \pi^{+} \longrightarrow \mu^{+}+\nu_{\mu} \quad \text { and } \quad \mu^{+} \longrightarrow e^{+}+\nu_{e}+\bar{\nu}_{\mu}, \\
& \pi^{-} \longrightarrow \mu^{-}+\bar{\nu}_{\mu} \text { and } \quad \mu^{-} \longrightarrow e^{-}+\bar{\nu}_{e}+\nu_{\mu}
\end{aligned}
$$

and $\gamma$-rays

$$
\pi^{0} \longrightarrow 2 \gamma
$$

In the decays 2-3 and 2-4, the muon leaves with approximately $\frac{3}{4}$ of the pion's energy, and shares its energy equally between its own decay products. This means that the each neutrino carries approximately one quarter of the initial charged pion energy. The photons produced via the neutral pion decay 2-5 also share the $\pi^{0}$ energy equally. An important observation is that, since IceCube does not distinguish between neutrinos and antineutrinos upon detection, we will only distinguish neutrino species by flavor. Hence, the flavor composition of the produced neutrinos is $\left(\nu_{e}: \nu_{\mu}: \nu_{\tau}\right)=(1: 2: 0) .^{3}$

Because of a scaling property of hadronic interactions [97], the neutrino and $\gamma$-ray spectra resulting from $p p$ scattering will have (almost) the same power-law slope as the initial protons. ${ }^{4}$ Moreover, different acceleration mechanisms at different energies predict a natural spectral break for neutrinos from $p p$ interactions $[98,99]$, which serves as justification for a us to adopt a broken power-law spectrum

${ }^{3}$ Due to the muon's relatively long lifetime $\left(\tau_{\mu}=2.2 \mu \mathrm{s}\right.$, compared to $\tau_{\pi^{ \pm}}=26 \mathrm{~ns}$ for charged pions), they can lose their energy before decaying by synchrotron radiation or multiple scattering in the dense astrophysical medium. If this muon damping process is dominant, the neutrino flavor ratio changes to $(0: 1: 0)$, and no electron neutrinos contribute to the high-energy neutrino flux.

${ }^{4}$ The widely accepted Fermi acceleration mechanism predicts $\sim E^{-2}$. 


$$
\frac{\mathrm{d} N_{\nu}}{\mathrm{d} \varepsilon_{\nu}}\left(\varepsilon_{\nu}\right) \propto \begin{cases}\varepsilon_{\nu}^{-s_{l}} & \varepsilon_{\nu}<\varepsilon_{\mathrm{br}} \\ \varepsilon_{\nu}^{-s_{h}} & \varepsilon_{\nu}>\varepsilon_{\mathrm{br}}\end{cases}
$$

where $\varepsilon_{\text {br }}$ marks a change in the spectral index. In particular, [99] argues that the neutrino production at galaxy clusters should obey $s_{l} \approx 2$ and $s_{h} \approx 2.5$, which is compatible with the IceCube observations if $\varepsilon_{\text {br }}$ is smaller than a few tens of $\mathrm{TeV}$ (this way, $\varepsilon_{\text {br }}$ corresponds to $E_{\text {th }}$ the IceCube fit in Eq. 2-1). However, this leads to multimessenger tensions with $\gamma$-ray data ${ }^{5}$ because $s_{l}$ is too soft (i.e. too large) [100], challenging the $p p$ scenario. $p \gamma$-induced processes, on the other hand, predict a harder $s_{l}$ and are favored with respect to the $p p$ case. This is subject of the next section.

\subsection{2}

\section{$p \gamma$ Scenario}

$p \gamma$ neutrino production most likely occurs inside the proton accelerators themselves, instead of CR reservoirs like in the $p p$ case. High luminosity objects like GRBs and AGN are good candidates for hosting such processes; they are also known to be powerful $\gamma$-ray sources and suggested to be where UHECRs are formed. The interaction of high energy protons with ambient photons results in resonant pion production (in the $\Delta$-resonance approximation), ${ }^{6}$

$$
p+\gamma \longrightarrow \Delta^{+} \longrightarrow \begin{cases}n+\pi^{+} & \frac{1}{3} \text { of the times } \\ p+\pi^{0} & \frac{2}{3} \text { of the times }\end{cases}
$$

which, after pion decay following Eqs. 2-3 - 2-5, yields the flavor ratio (1:2:0). Although this is the same as in the $p p$ case, there is a difference: $\pi^{-}$(and consequently $\bar{\nu}_{e}$ ) is not produced by these photohadronic interactions. ${ }^{7}$ This means that the differentiation between neutrino and antineutrino species could be a helpful discrimination between these two production mechanisms, which unfortunately IceCube is not capable of doing. In 2-7, pions receive approximately $20 \%$ of the proton's energy, which is distributed to their decay products the same way as described in the previous section.

A crucial property of $p \gamma$ interactions is that the resulting neutrino flux possesses a natural threshold from pion production kinematics (the neutrino carries $\approx 1 / 25$ of the proton's energy) at

$$
E_{\mathrm{th}}^{p \gamma} \approx \frac{1}{25} \frac{m_{\pi} m_{p}}{\epsilon_{t}} \approx 6 \times 10^{6} \mathrm{GeV}\left(\frac{\mathrm{eV}}{\epsilon_{t}}\right)
$$

${ }^{5}$ How we obtain such multimessenger constraints is described in detail in Chapter 4.

${ }^{6}$ At high energies $(\gtrsim 100 \mathrm{GeV})$, multipion production $p+\gamma \longrightarrow p+a \pi^{0}+b\left(\pi^{+}+\pi^{-}\right)$ dominates.

${ }^{7}$ This is a possible justification for the suppression of the Glashow resonance in the IceCube observations. 
where $\epsilon_{t}$ is the target photon's energy, below which the spectrum drops rapidly. The neutrino spectrum arising in this case can also be described by a broken power-law of the form 2-6, with $s_{l}=0$ reflecting this hardening below $\varepsilon_{\mathrm{br}} \sim E_{t h}^{p \gamma}$. Since there is no indication or preference towards having spectral breaks in the IceCube data, we can only expect that this break would be below the threshold energy for each dataset (see Section 2.1.2). In the most conservative scenario, this break can be set to the threshold energies themselves, which are between $\sim 10-100 \mathrm{TeV}$, but no higher. This means that there would be essentially no sub-TeV $\gamma$-ray emission from the sources. Also importantly, it has been shown in [100] that IceCube sources with $p \gamma$ origin are expected to be opaque to $\gamma$-rays in the $1-100 \mathrm{GeV}$ range, which can reduce the aforementioned multimessenger tension with $\gamma$-ray data in comparison to pp scenarios.

\subsection{3}

\section{$\nu-\gamma$ Connection}

In the previous sections, we have seen two scenarios for neutrino and $\gamma$-ray production in dense astrophysical environments. We have also seen how the emitted neutrino spectrum is expected to behave in each case. We are now left with the task of establishing a relation between the neutrino and $\gamma$ ray emission spectra, which is a crucial step in enabling our multimessenger analysis.

In summary, we have the energy relations

$$
\varepsilon_{\nu} \approx \frac{\varepsilon_{\pi^{ \pm}}}{4} \approx\left\{\begin{array}{ll}
\frac{\varepsilon_{p}}{12} & (p p) \\
\frac{\varepsilon_{p}}{20} & (p \gamma)
\end{array}, \quad \varepsilon_{\gamma} \approx \frac{\varepsilon_{\pi^{0}}}{2} \approx\left\{\begin{array}{ll}
\frac{\varepsilon_{p}}{6} & (p p) \\
\frac{\varepsilon_{p}}{10} & (p \gamma)
\end{array},\right.\right.
$$

which means that $\varepsilon_{\nu} \approx \varepsilon_{\gamma} / 2$ independently of the production mechanism. Additionally, for each charged pion we have 3 neutrinos produced, whereas for each neutral pion, 2 photons are generated. This means that the ratio between the number of neutrinos and photons emitted is $3 K_{\pi} / 2$, where $K_{\pi}$ is the ratio of charged to neutral pions produced in each process $\left(K_{\pi} \approx 1\right.$ for $p p$ and $K_{\pi} \approx 1 / 2$ for $p \gamma$, considering dominant channels only). Now we can finally relate the energy spectra of $\gamma$-rays to that of neutrinos:

$$
\left.\varepsilon_{\gamma}^{2} \frac{\mathrm{d} N_{\gamma}}{\mathrm{d} \varepsilon_{\gamma}}\left(\varepsilon_{\gamma}\right) \simeq \frac{2}{3 K_{\pi}} \varepsilon_{\nu}^{2} \frac{\mathrm{d} N_{\nu}}{\mathrm{d} \varepsilon_{\nu}}\left(\varepsilon_{\nu}\right)\right|_{\varepsilon_{\nu}=\varepsilon_{\gamma} / 2},
$$

where $\varepsilon_{\nu}^{2} \frac{\mathrm{d} N_{\nu}}{\mathrm{d} \varepsilon_{\nu}}\left(\varepsilon_{\nu}\right)$ is the all-flavor neutrino energy spectrum.

Eq. 2-10 is a conservative estimate to the $\gamma$-ray emission. We are neglecting $\gamma$-rays that arise from electrons and positrons that undergo bremsstrahlung or inverse Compton scattering inside the source, as well as ignoring neutrino 
suppression effects like radiative or adiabatic cooling of charged pions and muons. This conservative/minimalistic approach strengthens our results, since any tension found between the IceCube and Fermi data will certainly be larger in more realistic cases. On the other hand, we are also assuming that the sources are $\gamma$-ray transparent (i.e. all $\gamma$ rays produced in this model will escape the source successfully). If we assumed for example that the sources were completely opaque - this would mean we are facing a new class of sources never before observed - then there would be no $\gamma$-ray counterpart to the IceCube neutrinos, resulting in no tension with the Fermi data. We will return to discuss about the source's opacity at the end of Chapter 5.

\section{3}

\section{Neutrino Propagation}

As discussed in the Introduction, neutrinos are reliable messengers that are able to propagate through the Universe essentially undisturbed. Thankfully, this means that the physics of neutrino propagation from a distant source to the Earth is much simpler than for other cosmic messengers. We just need to account for the cosmological redshift in the neutrino's energy: a neutrino emitted with energy $\varepsilon_{\nu}$ from a source at redshift $z$ is detected here with energy $E_{\nu}=\varepsilon_{\nu} /(1+z)$.

Now consider a source with neutrino spectrum $\frac{\mathrm{d} N_{\nu}}{\mathrm{d} \varepsilon_{\nu}}\left(\varepsilon_{\nu}\right)$, in units of $\left[\mathrm{GeV}^{-1}\right.$ $\left.\mathrm{s}^{-1}\right]$. The corresponding neutrino flux at the Earth is given by (in units of $[\mathrm{GeV}$ $\left.\mathrm{cm}^{-2} \mathrm{~s}^{-1}\right]$ )

$$
E_{\nu}^{2} \Phi_{\nu}^{\mathrm{pt}-\text { source }}\left(E_{\nu}\right)=\frac{\left[\varepsilon_{\nu}^{2} \frac{\mathrm{d} N_{\nu}}{\mathrm{d} \varepsilon_{\nu}}\left(\varepsilon_{\nu}\right)\right]_{\varepsilon_{\nu}=(1+z) E_{\nu}}}{(1+z)^{2} 4 \pi d_{c}^{2}},
$$

where $d_{c}$ is the comoving distance of the source to the Earth.

We want to generalize this from a point source to a population of sources. For this, we require information about the distribution of these sources in the universe, which is encoded in the redshift-dependent source density $\mathcal{F}(z)$. Depending on the population of sources we are considering, $\mathcal{F}(z)$ takes a different shape (see Appendix B). By multiplying the point-source flux by the density of sources and integrating over all space, we obtain the contribution of all sources to the measured flux at the Earth. This integration is done by changing the integration variable from the comoving volume to redshift: $\mathrm{d} \mathcal{V}=\mathrm{d} z \frac{\mathrm{d} \mathcal{V}}{\mathrm{d} z}$. Finally, we divide by a factor of $4 \pi$ to get the desired units of $[\mathrm{GeV}$ $\mathrm{cm}^{-2} \mathrm{~s}^{-1} \mathrm{sr}^{-1}$, which is possible since the sources are isotropic, obtaining our desired relation 


$$
E_{\nu}^{2} \Phi_{\nu}^{\mathrm{diff}}\left(E_{\nu}\right)=\frac{1}{4 \pi} \int_{0}^{\infty} \mathrm{d} z \frac{\mathrm{d} \mathcal{V}}{\mathrm{d} z} \mathcal{F}(z) \frac{\left[\varepsilon_{\nu}^{2} \frac{\mathrm{d} N_{\nu}}{\mathrm{d} \varepsilon_{\nu}}\left(\varepsilon_{\nu}\right)\right]_{\varepsilon_{\nu}=(1+z) E_{\nu}}}{(1+z)^{2} 4 \pi d_{c}^{2}}
$$

We can further rewrite this expression in simpler terms by recalling Eqs. A-17 and A-18. A simple calculation results in

$$
\Phi_{\nu}^{\mathrm{diff}}\left(E_{\nu}\right)=\frac{1}{4 \pi} \int_{0}^{\infty} \mathrm{d} z \frac{\mathcal{F}(z)}{H(z)}\left[\frac{\mathrm{d} N_{\nu}}{\mathrm{d} \varepsilon_{\nu}}\left(\varepsilon_{\nu}\right)\right]_{\varepsilon_{\nu}=(1+z) E_{\nu}} .
$$

Eq. 2-13 represents the neutrino flux IceCube is measuring. We can use the IceCube data to set the overall normalization for this flux, compensating for our ignorance in the normalizations of the injection spectrum and source density. This is done in Section 4.2.3.

\subsection{1}

\section{Decoherent Neutrino Oscillations}

One last detail must be mentioned: even though the source emits neutrinos with uneven flavor distributions, the flavor composition of the neutrinos at the Earth is essentially $\left(\nu_{e}: \nu_{\mu}: \nu_{\tau}\right)=(1: 1: 1)$ due to decoherence in neutrino oscillations. We can understand this phenomenon using a quantum mechanical approach in which neutrino mass eigenstates are described by a wave packets and flavor eigenstates are superpositions of them [101].

Neutrinos interact as flavor eigenstates but propagate as mass eigenstates; these are related by the Pontecorvo-Maki-Nakagawa-Sakata (PMNS) matrix

$$
\left(\begin{array}{c}
\nu_{e} \\
\nu_{\mu} \\
\nu_{\tau}
\end{array}\right)=\left(\begin{array}{ccc}
U_{e 1} & U_{e 2} & U_{e 3} \\
U_{\mu 1} & U_{\mu 2} & U_{\mu 3} \\
U_{\tau 1} & U_{\tau 2} & U_{\tau 3}
\end{array}\right)\left(\begin{array}{c}
\nu_{1} \\
\nu_{2} \\
\nu_{3}
\end{array}\right)
$$

This matrix can be parametrized in terms of three mixing angles $\theta_{12}, \theta_{13}$ and $\theta_{23}$, as well as one CP-violating phase $\delta_{\mathrm{CP}}$ (assuming neutrinos are Dirac fermions) in the following way:

$$
U=\left(\begin{array}{ccc}
c_{12} c_{13} & s_{12} c_{13} & s_{13} e^{-i \delta_{\mathrm{CP}}} \\
-s_{12} c_{23}-c_{12} s_{13} s_{23} e^{i \delta_{\mathrm{CP}}} & c_{12} c_{23}-s_{12} s_{13} s_{23} e^{i \delta_{\mathrm{CP}}} & c_{13} s_{23} \\
s_{12} s_{23}-c_{12} s_{13} c_{23} e^{i \delta_{\mathrm{CP}}} & -c_{12} s_{23}-s_{12} s_{13} c_{23} e^{i \delta_{\mathrm{CP}}} & c_{13} c_{23}
\end{array}\right)
$$

where $c_{i j}\left(s_{i j}\right)$ is short-hand for $\cos \theta_{i j}\left(\sin \theta_{i j}\right)$. Because neutrinos are difficult to detect, the PMNS entries $U_{\alpha i}$ are much harder to determine than those from its quark equivalent, the Cabibbo-Kobayashi-Maskawa (CKM) matrix. The most recent results at the time of writing this thesis are displayed in Table 2.2. 
Table 2.2: Best-fit parameter values for the PMNS matrix following the parametrization in Eq. 2-15. The fit assumes normal ordering for the neutrino masses $\left(m_{1}<m_{2}<m_{3}\right)$ and includes data from solar, reactor, accelerator and atmospheric (including Super-Kamiokande) neutrino experiments [102, 103].

\begin{tabular}{c|c} 
& Best-fit Parameters $\pm 1 \sigma$ \\
\hline$\theta_{12}\left(^{\circ}\right)$ & $33.44_{-0.76}^{+0.78}$ \\
\hline$\theta_{13}\left(^{\circ}\right)$ & $8.57_{-0.12}^{+0.12}$ \\
\hline$\theta_{23}\left(^{\circ}\right)$ & $49.2_{-1.2}^{+0.9}$ \\
\hline$\delta_{C P}\left(^{\circ}\right)$ & $197_{-24}^{+27}$
\end{tabular}

In the wave-packet approach, each mass eigenstate travels with a different velocity. They eventually cease to overlap and interfere incoherently after a large enough distance. ${ }^{8}$ This coherence length is negligible when compared to the distance travelled by astrophysical neutrinos on their way to the Earth, which means that they arrive as an incoherent mixture of mass eigenstates. In this case, the flavor oscillation probability can be shown to be [104, 105]

$$
P\left(\nu_{\alpha} \longrightarrow \nu_{\beta}\right)=\sum_{i=1}^{3}\left|U_{\alpha i}\right|^{2}\left|U_{\beta i}\right|^{2}
$$

and, consequently, the flavor ratios at the Earth, $f^{\oplus}$, are related to the flavor ratios at the source, $f^{S}$, by

$$
f_{\nu_{\beta}}^{\oplus}=\sum_{\alpha=e}^{\tau} \sum_{i=1}^{3}\left|U_{\alpha i}\right|^{2}\left|U_{\beta i}\right|^{2} f_{\nu_{\alpha}}^{S} .
$$

Using the values from Table 2.2 in the parametrization 2-15 to calculate the $U_{\alpha i}$, we find that $\left(f_{\nu_{e}}^{\oplus}: f_{\nu_{\mu}}^{\oplus}: f_{\nu_{\tau}}^{\oplus}\right) \approx(1: 1: 1)$ for a source flavor ratio of $\left(f_{\nu_{e}}^{S}: f_{\nu_{\mu}}^{S}: f_{\nu_{\tau}}^{S}\right)=(1: 2: 0)$. Essentially, what this means for us is that the per-flavor neutrino flux at the Earth is a factor of 3 smaller than the all-flavor neutrino flux which we will be considering throughout this work.

This concludes Chapter 2 of this thesis. Next, we shall turn our attention to understanding the propagation of $\gamma$-rays, which is significantly more complicated than what we just saw for neutrinos. High energy photons undergo several interactions en route to us from their respective sources, initiating socalled "electromagnetic cascades". Chapter 3 is solely dedicated to describing this process in detail.

\footnotetext{
${ }^{8}$ This distance depends on the size of the wave-packets, the energy of the neutrino and on the mass differences between eigenstates [104].
} 


\section{3 \\ Electromagnetic Cascades}

The Universe is effectively opaque to photons with high enough energies. As we have seen in the Introduction (see Figure 1.1), $\gamma$-rays produced in astrophysical sources cannot travel long distances in intergalactic space without encountering an obstacle and interacting. This interaction initiates a process known as an "electromagnetic cascade" in which the initial $\gamma$-ray's energy is distributed to secondary particles which can propagate to and be detected at the Earth. In this chapter, we shall discuss $\gamma$-ray propagation in detail to understand why and how this phenomenon occurs.

The electromagnetic cascade (or simply "cascade") begins when a high energy $\gamma$-ray interacts with a background photon, producing an electronpositron pair. These particles then inverse Compton scatter off of background photons, transferring their energy to them and producing two new $\gamma$-rays. As long as the photons involved have sufficient energy to pair produce, this cycle repeats until we end up with several low energy photons, electrons and positrons. Of course, this is only a very superficial description of what happens, and the goal of this section is to explain the theory of $\gamma$-ray cascades by understanding in depth each step of the process.

Even though it is a complicated process, an analytic calculation of the cascaded $\gamma$-ray flux observed at the Earth is possible if we allow for several assumptions to be made:

(i) The cascade develops completely, or, in other words, there is no photon left with enough energy to pair produce;

(ii) The photon backgrounds are monochromatic;

(iii) Energy is conserved throughout all cascade particles (there is no energy loss through synchrotron radiation).

Useful reviews of this analytic calculation can be found at [106, 107, 108]. Nonetheless, if we want a more precise calculation of the cascaded flux that takes into account deviations from the aforementioned assumptions, we must 
resort to numerical methods. We shall briefly touch on how this can be done by the means of solving Boltzmann equations as presented in [107].

We shall begin this chapter with a description of the photon backgrounds pervading the Universe. Namely, these are the cosmic microwave background (CMB) and the extragalactic background light (EBL), both playing a crucial role in $\gamma$-ray cascade theory. Following that, we will discuss the details of how to calculate analytically the cascaded flux at the Earth given several simplifying assumptions. This approach divides the cascade into three regimes, each which shall be treated separately and thoroughly. Finally, a numerical approach will be briefly presented; briefly since this is not the focus of this thesis. Instead, we use the public code " $\gamma$-Cascade" [109] for precise calculations of the electromagnetic cascade.

\section{1}

\section{$\gamma$ Backgrounds}

\subsection{1}

\section{Cosmic Microwave Background}

The CMB consists of an omnipresent, nearly isotropic, ${ }^{1}$ blackbody-like background of relic photons from the epoch of recombination. Approximately 370,000 years after the Big Bang $(z \approx 1100)$, the Universe became cool enough ( $3000 \mathrm{~K})$ for electrons to pair with protons, forming the first Hydrogen atoms. Suddenly, photons who used to scatter off of free electrons became decoupled from matter and free to travel through space as they did not interact with neutral atoms. These are the photons which are currently observed to form the CMB at temperature [112]

$$
T_{c m b, 0}=2.72548 \pm 0.00057 \mathrm{~K}
$$

After its accidental discovery by A. Penzias and R. Wilson [3], the CMB has been studied in great detail, with the latest data acquisition and analysis having been done by the Planck Collaboration [113]. It is not the aim of this thesis to delve into too many details (we will certainly not be concerned with temperature or polarization anisotropies in this work); it suffices for us to know the energy and number density of the CMB photons. These values are, respectively, ${ }^{2}$

$$
\epsilon_{c m b, 0} \simeq 6.6 \times 10^{-4} \mathrm{eV}
$$

\footnotetext{
${ }^{1}$ Temperature variations are roughly of the order $\Delta T / T \sim 10^{-5}[110,111]$.

${ }^{2}$ This energy corresponds to the peak frequency of the CMB blackbody spectrum.
} 
and

$$
n_{c m b, 0} \simeq 410.5 \mathrm{~cm}^{-3} .
$$

This yields the energy density $u_{c m b, 0} \simeq 0.25 \mathrm{eV} \mathrm{cm}^{-3}$. Notice that these values are redshift dependent due to the expansion of the Universe: $\epsilon_{c m b}(z)=$ $\epsilon_{c m b, 0}(1+z), n_{c m b}(z)=n_{c m b, 0}(1+z)^{3}$ and $u_{c m b}(z)=u_{c m b, 0}(1+z)^{4}$.

\subsection{2}

\section{Extragalactic Background Light}

Optical/UV light emitted by stars and galaxies, as well as infrared light reprocessed in scattering with dust all contribute to another important background we will be considering in this work: the EBL. ${ }^{3}$ They are considerably less abundant than the CMB; so much that, even though they are more energetic (optical/UV in comparison to microwave radiation), their energy density satisfies $u_{e b l} \ll u_{c m b} .{ }^{4}$ The reason why we are concerned with the EBL is that, due to its higher energy photons, some processes which are characterized by high threshold energies (e.g. photon-photon pair production occurring in electromagnetic cascades) can be accessed earlier in the EBL than in the CMB.

Measuring the EBL requires observation of the sky in a wide range of wavelengths, using direct and indirect methods. Direct intensity measurements must take into account several foregrounds from the Solar System to the Milky Way, which can be especially challenging in certain frequency ranges. An example of an indirect measurement would be to use the absorption of $\mathrm{TeV}$ $\gamma$-rays from AGN via pair production over cosmological distances, which has yielded better results than direct methods in the optical range. A good review on the EBL intensity measurements from $\gamma$-rays to radio waves can be found in [114].

Unlike relic photons, the EBL does not possess a simple blackbody spectrum. In fact, its energy distribution at $z=0$ is closely approximated by three blackbody curves with two large peaks around $\lambda \sim 1 \mu \mathrm{m}$ and $\lambda \sim 100 \mu \mathrm{m}$ (commonly called the cosmic optical background (COB) and cosmic infrared background (CIB), respectively), and a smaller peak around $\lambda \sim 10 \mu \mathrm{m}$. Several models exist in literature [115, 116, 117, 118, 119]; the

\footnotetext{
${ }^{3}$ Sometimes, the term "EBL" refers to extragalactic background radiation with frequencies spread throughout the entire electromagnetic spectrum, from $\gamma$-rays to radio waves. The parts of the EBL we are interested range from infrared to UV light.

${ }^{4}$ Inside the galaxy, however, $u_{\text {starlight }} \gtrsim u_{c m b}$ (depending on how close you are to the galactic center).
} 
cascade simulation code we shall use in this work uses the Domínguez et al. model [115] which is shown in Figures 3.1 and 3.2.

Clearly, the EBL is hard to take into account when seeking to perform an analytical calculation of the electromagnetic cascade. A rather radical approach is to consider only the most energetic photons coming from $\lambda \lesssim 1 \mu \mathrm{m}$, although it is definitely not good enough for exact calculations. To accurately include the EBL in the cascade will require numerical simulations.

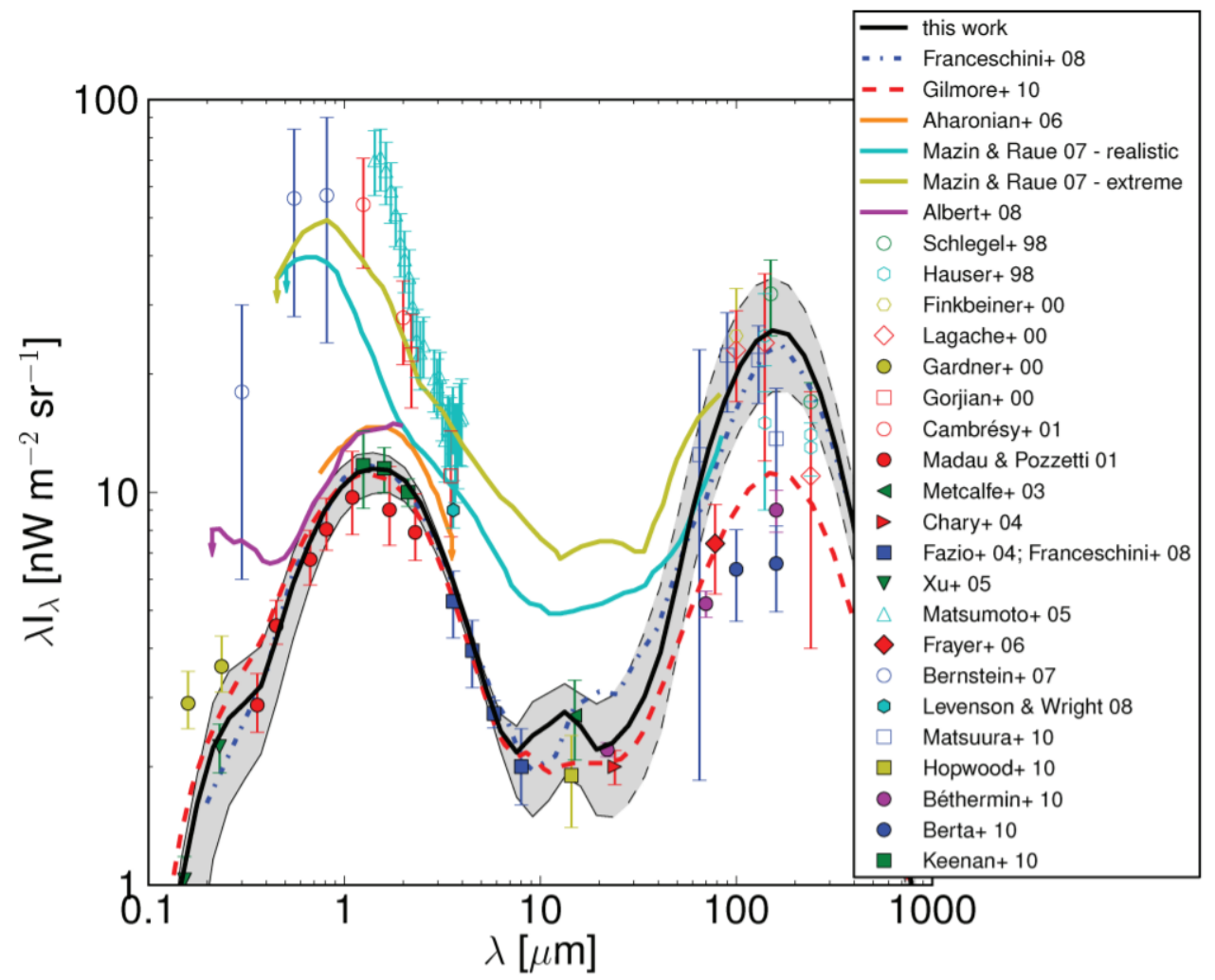

Figure 3.1: EBL intensity at $z=0$ as a function of wavelength as modelled by Domínguez et al [115]. The solid black line represents their EBL best fit with uncertainty given by the shaded gray region. Blue dot-dashed and red dashed lines correspond to other EBL models found in literature ([117] and [118] respectively). Colored solid lines are upper bounds from the study of blazars. Empty symbols are direct measurements while filled symbols are from galaxy counts, usually considered as lower limits. 


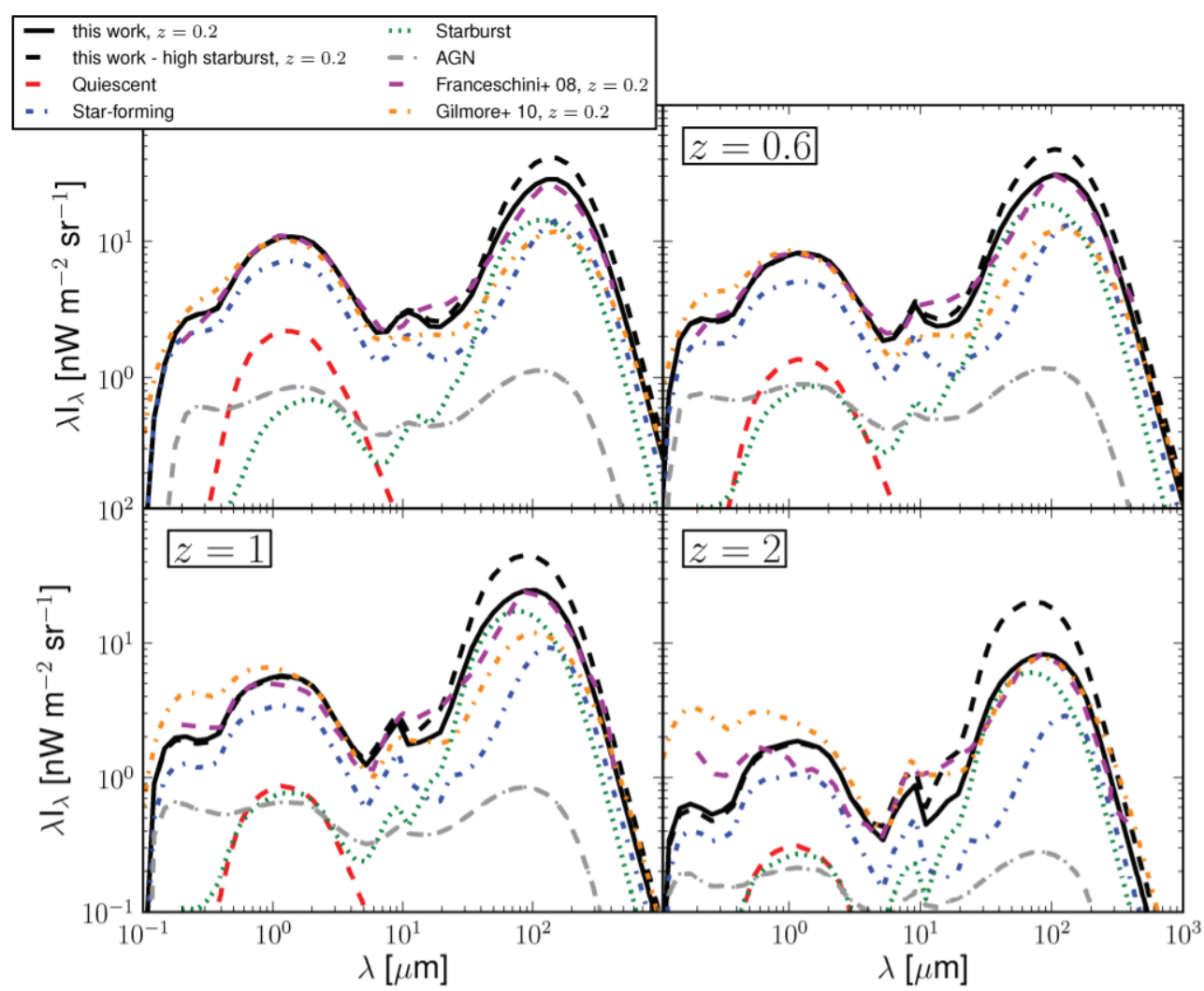

Figure 3.2: EBL evolution with redshift (top left panel: $z=0.2$ ), reproduced from [115]. The solid and dashed black lines represent their model under different assumptions for $z>1$ extrapolation (see the reference for details). Red dashed, blue dot-dashed, green dotted and gray dot-long-dashed lines represent contributions from quiescent, star-forming galaxies, starbursts and AGN, respectively. Magenta dashed and orange dot-dashed lines are from other models ([117] and [118] respectively), for comparison.

\section{2}

\section{Physical Processes in $\gamma$-ray Propagation}

Pair production (PP) and inverse Compton scattering (ICS) are the main processes involved in $\gamma$-ray propagation through space. ${ }^{5}$ In this section we will describe the physical features of both in order to understand how the cascade itself will develop, which is the topic of the next section. Useful reviews on these subjects can be found in [121] and [122]. For convenience, I shall henceforth refer to both electrons and positrons as simply "electrons", unless specified.

\footnotetext{
${ }^{5}$ Higher order processes like double and triple pair production become dominant at $\gamma$-ray energies above $E_{\gamma} \approx 10^{13} \mathrm{GeV}$, which is far beyond what is considered in this thesis [120].
} 


\subsection{1}

\section{Pair Production}

Electron-positron PP involving the collision of two photons, also known as the Breit-Wheeler process [123], is the first process we must understand in detail to fully address the electromagnetic cascade. It is represented by the reaction

$$
\gamma+\gamma_{t} \longrightarrow e^{+}+e^{-}
$$

where one of the initial photons is a low energy target (represented by the subscript $t$ ) and the other photon is a high energy $\gamma$-ray. In our case, the target will be a CMB or an EBL photon.

The first and most crucial aspect to notice is that there is a natural energy threshold below which pair production is no longer possible. It depends on the electron mass, $m_{e}$, and on the energy of the background/target photons, $\epsilon_{t}$ :

$$
E_{\gamma, t h}^{\mathrm{PP}}=m_{e}^{2} / \epsilon_{t}
$$

In the context of the cascade, this means that very high energy $\gamma$-rays $\left(E_{\gamma} \gtrsim\right.$ $400 \mathrm{TeV}$ ) pair-produce with both CMB and EBL backgrounds. However, as these $\gamma$-rays propagate and lose their energy, only EBL photons have sufficient energy to participate in PP. Eventually $\left(E_{\gamma} \lesssim 400 \mathrm{GeV}\right)$, both the CMB and the EBL become sterile for PP, resulting in a ICS-dominated cascade.

The differential cross section, in the frame in which the collision is collinear, for an incoming $\gamma$-ray with energy $E_{\gamma}$ interacting with a background photon with energy $\epsilon_{t}$, with respect to the outgoing electron's energy $E_{e}$, expressed in terms of the Thomson cross section, $\sigma_{\mathrm{T}}=8 \pi \alpha^{2} / 3 m_{e}^{2} \simeq 66.5$ $\mathrm{fm}^{2}$, is given by [120]

$$
\begin{aligned}
\frac{\mathrm{d} \sigma_{\mathrm{PP}}}{\mathrm{d} E_{e}}=\frac{3}{4} \sigma_{\mathrm{T}} \frac{m_{e}^{2}}{s E_{\gamma}}\left[\frac{E_{e}}{E_{\gamma}-E_{e}}+\right. & \frac{E_{\gamma}-E_{e}}{E_{e}}+E_{\gamma}\left(1-\beta^{2}\right)\left(\frac{1}{E_{e}}+\frac{1}{E_{\gamma}-E_{e}}\right)- \\
& \left.-\frac{E_{\gamma}^{2}\left(1-\beta^{2}\right)^{2}}{4}\left(\frac{1}{E-e}+\frac{1}{E_{\gamma}-E_{e}}\right)^{2}\right],
\end{aligned}
$$

where $s=4 E_{\gamma} \epsilon_{t}$ is the squared center of mass (CM) energy, $\beta=\sqrt{1-4 m_{e}^{2} / s}=$ $\sqrt{1-E_{\gamma, t h}^{\mathrm{PP}} / E_{\gamma}}$ is the velocity of the outgoing electron in the CM frame and the range is restricted to $\frac{1-\beta}{2} \leq \frac{E_{e}}{E_{\gamma}} \leq \frac{1+\beta}{2}$. We obtain the total cross section by integrating: 


$$
\sigma_{\mathrm{PP}}(\beta)=\frac{3}{16} \sigma_{\mathrm{T}}\left(1-\beta^{2}\right)\left[2 \beta\left(\beta^{2}-2\right)+\left(3-\beta^{4}\right) \ln \left(\frac{1+\beta}{1-\beta}\right)\right] .
$$

Cross section $3-7$ peaks at $\sigma_{\mathrm{PP}}^{\max } \approx \sigma_{\mathrm{T}} / 4$ for $E_{\gamma} \approx 2 E_{\gamma, t h}^{\mathrm{PP}}$. This means that PP on the $\mathrm{CMB}$ dominates for $\sim \mathrm{PeV} \gamma$-ray energies, while $\mathrm{COB}$ photons become the main targets for $E_{\gamma} \sim \mathrm{TeV}$.

We will be concerned with the behaviour in the high energy limit

$$
\sigma_{\mathrm{PP}} \stackrel{\beta \rightarrow 1}{\longrightarrow} \frac{3}{16} \sigma_{\mathrm{T}}\left(1-\beta^{2}\right)\left[2 \ln \left(\frac{4}{1-\beta^{2}}\right)-2\right] .
$$

For $\mathrm{PeV}$ photons interacting with the $\mathrm{CMB}$ or $\mathrm{TeV}$ photons interacting with the $\mathrm{COB}$, this approaches $\left(3 \sigma_{\mathrm{T}} / 16\right)$. We can estimate the mean free path $\lambda$ that $\gamma$-rays propagate before pair producing with targets of homogeneous density $n_{t}$ by

$$
\lambda=\left(n_{t} \sigma\right)^{-1}
$$

These numbers are displayed in Table 3.1 for targets from the COB and CMB, at $\mathrm{TeV}$ and $\mathrm{PeV} \gamma$-ray energies, respectively.

In each PP collision, one of the outgoing particles leaves with a fraction $f=E_{e} / E_{\gamma}$ of the incoming $\gamma$-ray's energy. At high energies, this fraction is

$$
f \approx 1-\left[\ln \left(2 E_{\gamma} \epsilon_{t} / m_{e}^{2}\right)\right]^{-1}, \quad \text { for } E_{\gamma} \gg E_{\gamma, t h}^{\mathrm{PP}},
$$

while at low energies, both $e^{ \pm}$share this energy equally,

$$
f \approx 0.5, \quad \text { for } E_{\gamma} \gtrsim E_{\gamma, t h}^{\mathrm{PP}} .
$$

This behaviour will divide the pair-producing stage of the cascade into two parts: $(i)$ At high energies, only one leading particle will carry away most of the energy of the initial $\gamma$-ray (from Eq. 3-10, $f \rightarrow 1$ as $E_{\gamma} \rightarrow \infty$ ), while the other low-energy particle leaves the cascade (or starts a new one at a much lower energy). (ii) At lower $\gamma$-ray energies, the outgoing particles share equally the total incoming energy, doubling the number of particles in the cascade in each PP step.

\subsection{2}

\section{Inverse Compton Scattering}

Compton scattering can be succinctly represented by the following scattering reaction: $e+\gamma \longrightarrow e^{\prime}+\gamma^{\prime}$. However, this representation misses a key feature that qualitatively differentiates regular Compton scattering from the process we are actually interested in, which is inverse Compton scattering. Regular Compton scattering refers an energy transfer from the photon to a target electron, resulting in an increase in the photon's wavelength. ICS is 
precisely the opposite: a high energy electron scatters off of a target photon, transferring energy to it. Hence, a subtle change in the reaction's equation better represents this process as

$$
e+\gamma_{t} \longrightarrow e^{\prime}+\gamma^{\prime}
$$

Similarly to PP, the target photons come from either the CMB or the EBL.

To lowest order (i.e. in tree-level QED), a very similar derivation to Eq. 3-7 (since this process is related to PP through crossing symmetry, it is actually the same derivation, simply changing the 4-momenta) yields the Klein-Nishina formula for the Compton scattering differential cross section in the electron rest frame [124]

$$
\frac{\mathrm{d} \sigma_{\mathrm{ICS}}}{\mathrm{d} \Omega}=\frac{\alpha^{2}}{2 m_{e}^{2}}\left(\frac{E_{\gamma^{\prime}}}{E_{\gamma}}\right)^{2}\left[\frac{E_{\gamma^{\prime}}}{E_{\gamma}}+\frac{E_{\gamma}}{E_{\gamma^{\prime}}}-\sin ^{2} \theta\right],
$$

where $\theta$ is the scattering angle and $\frac{E_{\gamma^{\prime}}}{E_{\gamma}}$ is the ratio between the final and initial photon energies. Given $E_{\gamma}$, we can obtain $E_{\gamma^{\prime}}$ from the well known Compton scattering relation

$$
E_{\gamma^{\prime}}=E_{\gamma}\left[1+\frac{E_{\gamma}}{m_{e}}(1-\cos \theta)\right]^{-1},
$$

which adds another $\theta$-dependence into the differential cross section.

It is worthwhile noting that, in the low-energy limit $E_{\gamma} \ll m_{e}$ (or, equivalently, $\frac{E_{\gamma^{\prime}}}{E_{\gamma}} \rightarrow 1$ from Eq. 3-14), Eq. 3-13 reduces to the familiar Thomson scattering cross section:

$$
\frac{\mathrm{d} \sigma_{\mathrm{ICS}}}{\mathrm{d} \Omega} \stackrel{E_{\gamma} \ll m_{e}}{\longrightarrow} \frac{\alpha^{2}}{2 m_{e}^{2}}\left(1+\cos ^{2} \theta\right) \Rightarrow \sigma_{\mathrm{ICS}} \stackrel{E_{\gamma} \ll m_{e}}{\longrightarrow} \frac{8 \pi \alpha^{2}}{3 m_{e}^{2}}=\sigma_{\mathrm{T}} .
$$

In switching from the lab (primed) frame, where the electron has Lorentz factor $\gamma_{e}=\left(1-\beta_{e}^{2}\right)^{-1 / 2}=E_{e}^{\prime} / m_{e}$ and travels at an angle $\theta^{\prime}$ with respect to the target photon, to the electron rest frame which we have been considering, the photon energy transforms as

$$
E_{\gamma}=E_{\gamma}^{\prime} \gamma_{e}\left(1-\beta_{e} \cos \theta^{\prime}\right) .
$$

Hence, the Thomson limit in the lab frame can be expressed as $E_{\gamma}^{\prime} E_{e}^{\prime} \ll m_{e}^{2}$. This does not reflect the ICS regime for $\mathrm{PeV}$ cascade electrons (or even $\mathrm{TeV}$ electrons interacting with the EBL).

In fact, we are interested in the Klein-Nishina limit, which is the opposite case: $E_{\gamma} E_{e} \gg m_{e}^{2}$ (the prime subscript is dropped as we will only work in the lab frame from now on). The cross section now behaves as [124]

$$
\sigma_{\mathrm{ICS}} \stackrel{E_{\gamma} E_{e} \gg m_{e}^{2}}{\longrightarrow} \frac{3}{8} \sigma_{\mathrm{T}} \frac{m_{e}^{2}}{E_{\gamma} E_{e}} \ln \left(\frac{2 E_{\gamma} E_{e}}{m_{e}^{2}}\right),
$$


which is $\approx \sigma_{\mathrm{T}} / 4$ for ICS of $\mathrm{PeV}$ electrons with the CMB for TeV electrons on COB photons. This results in a mean free path of $\sim 10 \mathrm{kpc}$ for the former and $\sim 1 \mathrm{Mpc}$ for the latter. This cross section decreases as we go deeper into the Klein-Nishina regime: it is $\sim 10^{-3} \sigma_{\mathrm{T}}$ for a $\mathrm{PeV}$ electron scattering with the COB. For comparison, Table 3.1 includes the mean free path of regular Compton scattering, which is $\approx 6$ orders of magnitude larger than the size of the observable universe.

Equation 3-10 (replacing $E_{\gamma}$ by the incoming electron's energy $E_{e}$ ) is also valid for ICS at high energies $\left(E_{e} \gg E_{\gamma, t h}^{\mathrm{PP}}\right)$, which means that the final photon leaves with most of the incoming electron's energy. ${ }^{6}$ The same applies for $E_{e} \sim E_{\gamma, t h}^{\mathrm{PP}}: f \approx 0.5$. At lower energies where $\mathrm{PP}$ is no longer possible $\left(E_{e} \ll E_{\gamma, t h}^{\mathrm{PP}}\right.$ ), if $\epsilon_{t} \ll m_{e}$ (which is true for both the CMB and the EBL), then the outgoing photon from ICS carries a fraction of the electron's energy [122]

This means that

$$
f=\frac{4}{3} E_{e} \epsilon_{t} / m_{e}^{2}, \quad \text { for } E_{e} \ll E_{\gamma, t h}^{\mathrm{PP}} .
$$

$$
E_{\gamma^{\prime}}=\frac{4}{3} \gamma_{e}^{2} \epsilon_{t}, \quad \text { for } E_{e} \ll E_{\gamma, t h}^{\mathrm{PP}},
$$

where $\gamma_{e}=E_{e} / m_{e}$ is the Lorentz factor of the electron. In fact, Eq. 3-19 is an approximation used in the analytical cascade calculations; deviations from the exact relation become significant above $E_{e} \approx 3 \times 10^{13} \mathrm{eV}$ (see Figure 2 in $[106])$.

Table 3.1: Summary of PP, ICS and regular Compton scattering cross sections and order-of-magnitude mean free paths for different incoming particle energies, adapted from [107]. The target photons come from either the COB (optical part of the EBL) or the CMB. Their respective densities are also displayed in the table. The mean free path for regular Compton scattering is shown for comparison.

\begin{tabular}{c|c|c|c} 
Process & Target Density $\left[\mathbf{c m}^{-3}\right]$ & Cross Section $\left[\sigma_{T}\right]$ & $\lambda[\mathbf{M p c}]$ \\
\hline$\gamma(\mathrm{TeV})+\gamma_{c o b} \longrightarrow e^{+}+e^{-}$ & 0.5 & $3 / 16$ & 10 \\
\hline$\gamma(\mathrm{PeV})+\gamma_{c m b} \longrightarrow e^{+}+e^{-}$ & 410.5 & $3 / 16$ & $10^{-2}$ \\
\hline$e(\mathrm{TeV})+\gamma_{c o b} \longrightarrow e^{\prime}+\gamma^{\prime}$ & 0.5 & $1 / 4$ & 10 \\
\hline$e(\mathrm{PeV})+\gamma_{c m b} \longrightarrow e^{\prime}+\gamma^{\prime}$ & 410.5 & $1 / 4$ & $10^{-2}$ \\
\hline$e(\mathrm{PeV})+\gamma_{c o b} \longrightarrow e^{\prime}+\gamma^{\prime}$ & 0.5 & $10^{-3}$ & $10^{3}$ \\
\hline$\gamma+e_{t} \longrightarrow \gamma^{\prime}+e^{\prime}$ (regular Compton) & $10^{-7}$ & $10^{-3}$ & $10^{10}$
\end{tabular}

${ }^{6}$ In ICS, we implicitly redefine $f$ to be $E_{\gamma} / E_{e}$. 


\section{3}

\section{Cascade Regimes}

Let us now work towards uniting PP and ICS into a single picture. As a simplifying assumption, we will consider in this section the photon target background to be dichromatic, with energies $\epsilon_{c m b}=6.6 \times 10^{-4} \mathrm{eV}$ and $\epsilon_{e b l}=0.68 \mathrm{eV}$ (corresponding to the COB peak at $\approx 1.8 \mu \mathrm{m}$ ). This is an approximation we must make in order to make analytic calculations about the behaviour of cascades. However, one must always remember that the CMB is actually given by a blackbody spectrum and the EBL is even more complicated to model (see Sec. 3.1.2). For a description of a simpler monochromatic cascade, see the Appendix in [108].

These assumptions will divide the cascade into three regimes. Transitions between these regimes occur when the cascade particles approach PP threshold with the CMB and EBL:

$$
\begin{gathered}
E_{t h, c m b}=m_{e}^{2} / \epsilon_{c m b} \approx 4.0 \times 10^{14} \mathrm{eV}, \\
E_{t h, e b l}=m_{e}^{2} / \epsilon_{e b l} \approx 3.8 \times 10^{11} \mathrm{eV} .
\end{gathered}
$$

Let us now describe each regime separately by following the energy evolution of the cascade (instead of its evolution in time).

\subsection{1}

\section{Leading Particle Regime}

After the initial $\gamma$-ray has left its source, it will eventually find a background photon to interact with, producing an $e^{ \pm}$pair. One of these particles will receive almost all of the $\gamma$-ray's energy and eventually inverse Compton scatter off of another background photon. By comparing densities $\left(n_{c m b} \gg n_{e b l}\right)$, we can expect CMB photons to be the main targets in the $E>E_{t h, c m b}$ stages of the cascade. So far, the cascade is oblivious to the presence of the EBL.

In each collision (PP or ICS), the incoming leading particle (i.e. the most energetic particle) transfers a large fraction $f \approx 1-\left[\ln \left(2 E \epsilon_{c m b} / m_{e}^{2}\right)\right]^{-1} \approx 1$ of its initial energy $E$ to the next leading particle, either the outgoing photon in ICS or an electron from PP. The non-leading particle, which is always an electron, leaves with $f E$ and starts a new cascade at a considerably lower energy. This consecutive $\mathrm{PP} \rightarrow \mathrm{ICS} \rightarrow \mathrm{PP} \rightarrow \ldots$ chain repeats itself until the leading particle approaches the energy threshold for pair production with the CMB, $E_{t h, c m b}$, given by Eq. 3-20.

A transition to the next regime begins at $E \approx E_{t h, c m b}$ (in reality, it begins 
a bit earler at $\left.\approx 5 E_{t h, c m b}\right)$, where $f \approx 0.5$ for CMB collisions means there is no longer one leading particle, but instead all products share equal energies. Nevertheless, non-leading electrons can always start this next regime early if $f E$ is small enough.

\subsection{2}

\section{Multiplication Regime}

When PP with the CMB is no longer possible, EBL photons take over this process and pair production is now exclusively $\gamma+\gamma_{e b l} \longrightarrow e^{+}+e^{-}$. Meanwhile, ICS targets still come mostly from the CMB since they are more numerous than the EBL. The energy loss fraction being $\approx 0.5$ for ICS with the $\mathrm{CMB}$ (at $E_{e} \approx E_{t h, c m b}$ ) and for PP with the EBL (at $E_{\gamma} \gtrsim E_{t h, e b l}$ ) means that the products in each collision share equal energies. With each step, the number of particles participating in the cascade increases; hence the name "multiplication regime".

This regime ends at the PP threshold energy with the EBL, $E_{t h, e b l}$ from Eq. 3-21, formally marking the end of the cascade. The minimum energy of cascade photons in this regime is therefore $E_{t h, e b l}$, and the minimum energy of cascade electrons coming from these threshold pair-producing photons is $E_{t h, e b l} / 2$.

\subsection{3}

\section{Low Energy Regime}

After PP is no longer possible, no new electrons are produced and ICS on $\mathrm{CMB}$ photons dominates because there are leftover electrons from the multiplication regime. Photons resulting from ICS at these energies $\left(E_{e} \ll\right.$ $\left.E_{t h, c m b}\right)$ are produced with energy according to Eq. 3-19. Notably, if one considers an electron leaving the multiplication regime with the lowest possible energy, $E_{e}=E_{t h, e b l} / 2$, then the corresponding Compton scattered photon will have energy.

$$
E_{X}=\frac{4}{3} \gamma_{e}^{2} \epsilon_{c m b}=\frac{1}{3} m_{e}^{2} \frac{\epsilon_{c m b}}{\epsilon_{e b l}^{2}} \approx 1.2 \times 10^{8} \mathrm{eV} .
$$

All photons produced with $E_{\gamma}<E_{t h, e b l}$ are said to be "cascade-sterile" from now on. A pictorial summary of all that has been discussed so far regarding the three cascade regimes can be found in Figure 3.3.

\footnotetext{
${ }^{7}$ It is worth mentioning that the EBL radiation is absent at $z \gg 1$, which means that this second stage of the cascade can only begin at smaller redshifts $(z \sim 2-3)$.
} 


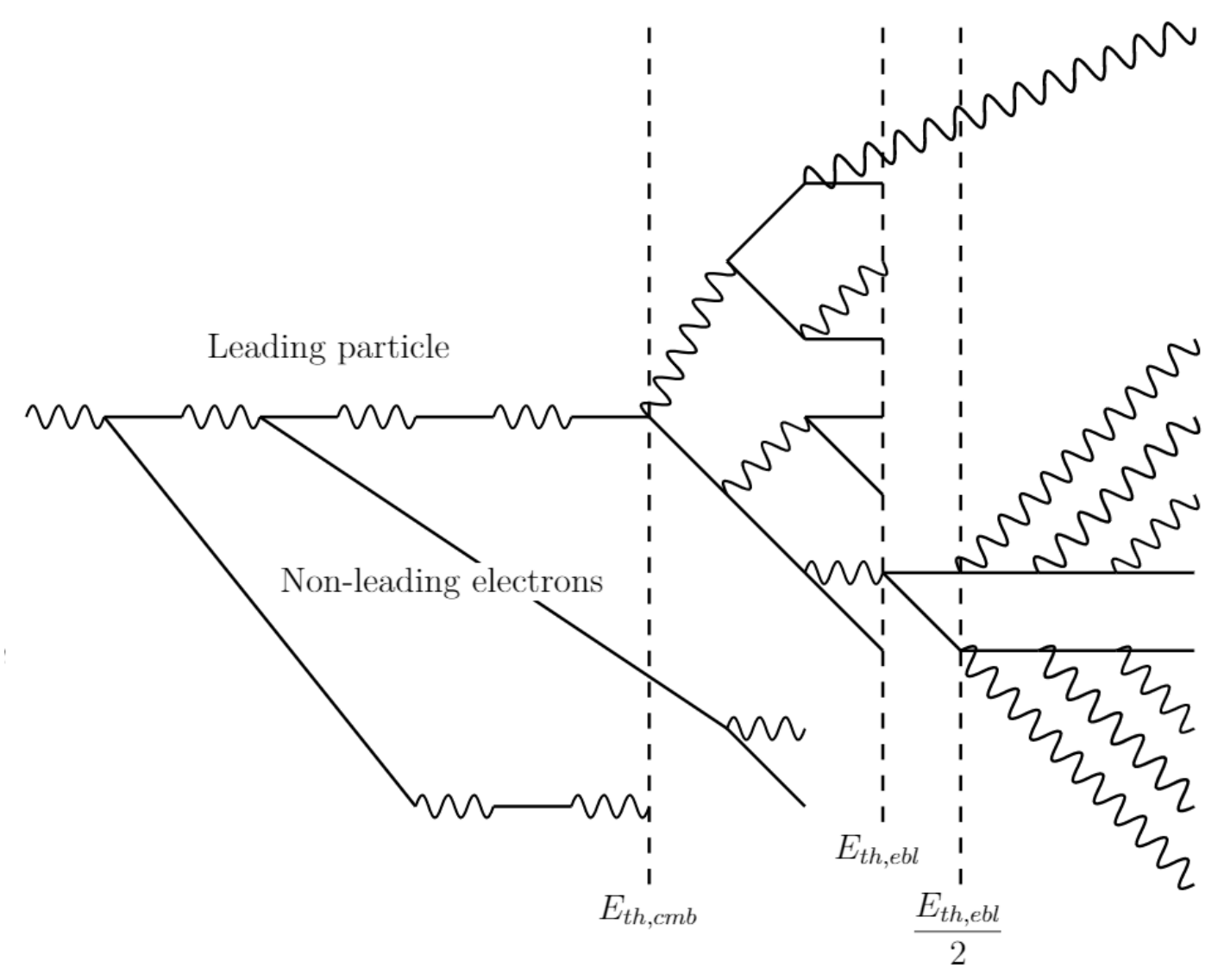

Figure 3.3: Illustrative representation of an electromagnetic cascade, adapted from [107]. The standard notation of curly lines for photons and straight solid lines for electrons is adopted. From left to right, the vertical dashed lines separate the leading particle, multiplication and low energy regimes. In the leading particle regime, examples of non-leading electrons which start new cascades at $E>E_{t h, c m b}$ and $E_{t h, c m b}>E>E_{t h, e b l}$ are shown. In the multiplication regime, the uppermost photon is an example of a photon produced with energy $E_{t h, e b l}>E_{\gamma}>E_{X}$, becoming cascade-sterile since it cannot pair produce.

\section{4}

\section{Spectral Features of Cascaded $\gamma$-rays}

After considering the kinematics and energetics of the cascade processes, we now want to characterize the remnant $\gamma$-ray spectrum arising from it. Notably, in the dichromatic model we have considered, this spectrum has a universal shape (independent of the initial energy of the primary photon, $E_{\text {tot }}$, or injection spectrum $\frac{\mathrm{d} N_{\gamma}}{\mathrm{d} E_{\gamma}}\left(E_{\gamma}\right)$ entering the cascade, assuming that $\left.E_{t o t}>E_{t h, e b l}\right)$ with characteristic energies that mark changes in its behavior: $E_{t h, e b l}$ and $E_{X}$.

Let's introduce a new quantity: $q(E)$ denotes the number of cascade particles passing through energy $E$ during the entire duration of the cascade. The notation for photons and electrons is $q_{e}(E)$ and $q_{\gamma}(E)$, respectively, 
satisfying

$$
q_{e}(E)+q_{\gamma}(E)=q(E) .
$$

We make two assumptions in the arguments that follow: $(i)$ the cascade develops completely, and ( $i i)$ energy is conserved throughout the entire cascade.

As a consequence of these assumptions, $E q(E)=E_{t o t}$ is a fixed value for any $E$. It is also easy to see that $q_{e}(E)=$ const. for $E<E_{t h, e b l} / 2$, since no electrons are produced in the low energy regime, and the existing ones will gradually lose all their energy by radiating Compton photons. In the multiplication regime, since the number of cascade electrons and photons are related by $N_{e} \approx 2 N_{\gamma}{ }^{8}$ energy conservation leads us to $E q_{e}(E)=2 E q_{\gamma}(E)$. Together with Eq. 3-23, we find that $q_{e}(E)=(2 / 3) E_{\text {tot }} / E=2 q_{\gamma}(E)$. Most importantly, the energy dependence $q_{e}(E) \propto E^{-1}$ in the multiplication regime will be important in what follows.

Given a single electron with energy $E_{e}$, it radiates $\mathrm{d} E_{e} / E_{\gamma}$ photons with energy $E_{\gamma}=f E_{e}$ in its deceleration to energy $E_{e}-\mathrm{d} E_{e} \cdot{ }^{9}$ Since $q_{e}\left(E_{e}\right)$ electrons pass through energy $E_{e}$ during the cascade, then the total number of photons emitted with $E_{\gamma}$ is

$$
\mathrm{d} N_{\gamma}\left(E_{\gamma}\right)=q_{e}\left(E_{e}\right) \mathrm{d} E_{e} / E_{\gamma}
$$

In the low energy regime, the energy spectrum of cascade-sterile photons can be obtained by substituting $q_{e}\left(E_{e}\right)=$ const. and $E_{\gamma} \propto E_{e}^{2}$ (from Eq. 3-19) into Eq. 3-24, leading to the low energy asymptotic behaviour

$$
\frac{\mathrm{d} N_{\gamma}}{\mathrm{d} E_{\gamma}} \propto E_{\gamma}^{-3 / 2}, \quad \text { for } E_{\gamma} \leq E_{X} .
$$

In the end of the multiplication regime, photons that are produced between $E_{X} \leq E_{\gamma} \leq E_{t h, e b l}$ are not absorbed by backgrounds and become cascadesterile. We can use $q_{e}\left(E_{e}\right) \propto E_{e}^{-1}$ and $E_{\gamma} \propto E_{e}^{2}$ (which is true since ICS occurs in the $\mathrm{CMB}$ ) to find

$$
\frac{\mathrm{d} N_{\gamma}}{\mathrm{d} E_{\gamma}} \propto E_{\gamma}^{-2}, \quad \text { for } E_{X} \leq E_{\gamma} \leq E_{t h, e b l} .
$$

Finally, as long as PP is still possible (in either the CMB or the EBL), all photons eventually transform into $e^{ \pm}$pairs and $\frac{\mathrm{d} N_{\gamma}}{\mathrm{d} E_{\gamma}}=0$.

Gathering all of these results, we can express the cascaded photon spectrum for a point-like source in the following way:

${ }^{8}$ One can work out the exact relations to be $N_{e}=2 N_{\gamma}-2(-1)^{\nu}$ for a photon entering the multiplication regime and $N_{e}=2 N_{\gamma}+(-1)^{\nu}$ for an electron entering the multiplication regime, after $\nu$ generations.

${ }^{9}$ Think of this as sharing $\mathrm{d} E_{e}$ among $\mathrm{d} N$ photons of energy $E_{\gamma}$. Then $\mathrm{d} E_{e}=\mathrm{d} N \times E_{\gamma}$. 


$$
\frac{\mathrm{d} N_{\gamma}}{\mathrm{d} E_{\gamma}}\left(E_{\gamma}\right)= \begin{cases}\left(K / E_{X}\right)\left(E_{\gamma} / E_{X}\right)^{-3 / 2} & \text { for } E_{\gamma} \leq E_{X} \\ \left(K / E_{X}\right)\left(E_{\gamma} / E_{X}\right)^{-2} & \text { for } E_{X} \leq E_{\gamma} \leq E_{t h, e b l} \\ 0 & \text { for } E_{\gamma}>E_{t h, e b l}\end{cases}
$$

where $K$ is a normalization constant. It can be found by energy conservation,

$$
\int_{0}^{\infty} E_{\gamma} \frac{\mathrm{d} N_{\gamma}}{\mathrm{d} E_{\gamma}} \mathrm{d} E_{\gamma}=E_{t o t}
$$

resulting in

$$
K=\frac{E_{t o t}}{E_{X}\left[2+\ln \left(E_{t h, e b l} / E_{X}\right)\right]} .
$$

This is the only place where the total energy injected into the cascade, $E_{t o t}$, appears in the resulting spectrum. This is why Eq. 3-27 is said to reflect a universal spectrum, frozen at the remnant photons stage (independent of the production stage).

The spectrum 3-27 is not valid for sources too close or too far from us. If they are too close $\left(r_{\text {source }} \ll\left(\sigma_{\mathrm{PP}} n_{e b l}\right)^{-1}\right)$, photon absorption on the EBL may not occur due to the small density of EBL targets (we have been neglecting target density so far because of the assumption that the cascade develops completely). In this case the cascade can occur with only the CMB; see [106] for the implications.

For high- $z$ sources, the effect of cosmological redshift becomes important. The energy of CMB photons evolves as $\epsilon_{c m b}(z)=(1+z) \epsilon_{c m b, 0}$, while the EBL varies nontrivially (see Figures 3.1 and 3.2$), \epsilon_{e b l}(z)$, because the evolution of its sources also come into play. This reflects in slight modifications to the relevant energies for the universal spectrum:

and

$$
E_{t h, e b l}(z)=\frac{m_{e}^{2}}{\epsilon_{e b l}(z)}
$$

$$
E_{X}(z)=\frac{m_{e}^{2} \epsilon_{c m b}(z)}{3\left(\epsilon_{e b l}(z)\right)^{2}} .
$$

At the Earth, these energies are redshifted by a factor of $(1+z)$ :

and

$$
E_{t h, e b l}^{\oplus}(z)=\frac{m_{e}^{2}}{(1+z) \epsilon_{e b l}(z)}
$$

$$
E_{X}^{\oplus}(z)=\frac{m_{e}^{2} \epsilon_{c m b, 0}}{3\left(\epsilon_{e b l}(z)\right)^{2}} .
$$

The new spectrum now has an additional $z$-dependence, and $K$ can be found by particle number conservation, $\frac{\mathrm{d} N_{\gamma}}{\mathrm{d} E_{\gamma}}\left(E_{\gamma}, z\right) \mathrm{d} E_{\gamma}=\frac{\mathrm{d} N_{\gamma}}{\mathrm{d} E_{\gamma}}\left(E_{\gamma}, 0\right) \mathrm{d} E_{\gamma}$. Still, Eq. 3-27 
remains valid besides the changes in the values of its characteristic energies.

Needless to say, in more realistic setups, the sharpness of the spectral features in Eq. 3-27 are smoothed out. Namely, there is a transition region around $E_{X}$ where the spectral index changes smoothly from $E^{-3 / 2}$ to $E^{-2}$, and the cutoff at $E_{t h, e b l}$ is not sharp, mainly because the EBL is not monochromatic and the cascade does not necessarily develop completely.

\section{5}

\section{Numerical Cascade Simulations}

Real cascades can produce spectra far from what we have seen so far in this chapter. We have briefly discussed the effect of cosmological redshift for high- $z$ sources, but low- $z$ sources remain a problem because the cascade might not have enough time to develop completely. Radiative energy loss processes such as synchrotron emission by electrons have also been neglected so far, as well as the energy distribution of CMB and EBL photons. In this section, we briefly outline how to take into account such effects in numerical simulations. We will refrain from discussing numerical techniques to solve the cascade transport equations; the reader is directed to [107] for more information on the subject.

The basic idea behind numerically simulating an electromagnetic cascade is following its time evolution, in contrast to our previous discussion regarding the evolution in energy. This approach will lead us to an integro-differential equation, which can be solved numerically using the Runge-Kutta technique for example.

Consider an isotropic distribution of incident particles with distribution in energy $N_{i}\left(E_{i}\right)$ scattering off targets with density distribution $n_{t}\left(\epsilon_{t}\right)$. This scattering process is characterized by the differential cross section $\frac{\mathrm{d} \sigma}{\mathrm{d} E^{\prime}}\left(E, E^{\prime}\right)$ for an incoming particle with energy $E$ producing a particle with energy $E^{\prime}$. Let $\mu=\cos \theta$, where $\theta$ is the scattering angle, and $\beta$ be the velocity of the incoming particle in the lab frame. The production of particles from this process is given by

$$
\dot{N}=c \int_{\epsilon_{t, \min }}^{\epsilon_{t, \max }} n_{t}\left(\epsilon_{t}\right) \mathrm{d} \epsilon_{t} \int_{-1}^{1} \frac{1-\beta \mu}{2} \mathrm{~d} \mu \int_{E_{i, \min }}^{E_{i, \max }} N_{i}\left(E_{i}\right) \mathrm{d} E_{i} \int_{E_{\min }}^{E_{\max }} \frac{\mathrm{d} \sigma}{\mathrm{d} E^{\prime}}\left(E, E^{\prime}\right) \mathrm{d} E .
$$

Targets in our case are CMB and EBL photons. Since the CMB is thermal, we can model it by a Planck distribution with peak temperature $T_{c m b}(z)=T_{c m b, 0}(1+z)$,

$$
n_{c m b}(\epsilon) \mathrm{d} \epsilon=\frac{1}{\pi^{2}} \frac{\epsilon^{2} \mathrm{~d} \epsilon}{\exp \left(\frac{\epsilon}{k_{b} T_{c m b}(z)}\right)-1} .
$$


Many models exist for EBL photons in literature, but most treat the CIB and COB as two blackbody curves (see Sec. 3.1.2).

To consider all energy loss processes in the cascade, we make use of a steady state equation known as transport or kinetic equation. Given an injection spectrum $N_{i n}(E)$, this equation allows us to calculate the observed spectrum $N(E)$ after the cascade. For electrons, one must take into account a continuous, isotropic injection from the sources, $\dot{N}_{e, i n}$, the loss through ICS, $\dot{N}_{e, i c s}$, and production via PP, $\dot{N}_{e, p p}$. Both $\dot{N}_{e, p p}$ and $\dot{N}_{e, i c s}$ have the form in Eq. 3-34, with cross sections given by Eqs. 3-7 and 3-13, respectively. Gathering these contributions,

$$
\dot{N}_{e}=\dot{N}_{e, i n}-\dot{N}_{e, i c s}+\dot{N}_{e, p p} .
$$

It is common to consider $\dot{N}_{e, i n}=0$ since most astrophysical sources do not allow electrons to escape because of intense magnetic fields.

We can incorporate an additional term due to continuous energy loss along the propagation direction $x$,

$$
\frac{\mathrm{d} N}{\mathrm{~d} x}(E)=-\alpha N(E)+j
$$

which can be included to model synchrotron radiation or $\gamma \gamma$ absorption in dense environments, for example. In 3-37, $\alpha$ is the coefficient of energy loss and $j$ is the source/injection term. The full kinetic equation for $\gamma$-rays reads

$$
\dot{N}_{\gamma}=\dot{N}_{\gamma, i n}+\dot{N}_{\gamma, i c s}-\dot{N}_{\gamma, p p}-c \frac{\mathrm{d} N_{\gamma}}{\mathrm{d} x} .
$$

Keep in mind that each term in this equation is either of the form 3-34 or 3-37. Due to its complexity, it can only be solved numerically, which we will abstain from doing here. Instead, we will move on to briefly describing the specific cascade simulation method used in this thesis.

\subsection{1}

\section{$\gamma$-Cascade Simulations}

The code used in this work for cascade simulation is the publicly available Mathematica package " $\gamma$-Cascade" [109]. Although we only use it for static (injection spectrum independent of redshift $z$ ) source distributions, it also simulates $\gamma$-ray propagation for point sources and evolving source populations (injection spectrum with a $z$-dependence). As an input, we provide two characteristics about the sources: their $\gamma$-ray injection spectrum, $\left.\frac{\mathrm{d} N_{\gamma}}{\mathrm{d} E_{\gamma}}\right|_{\text {inj }}$, and their spatial density distribution $\mathcal{F}(z)$ (assumed to follow the SFR, see Appendix B). After running the calculations briefly outlines in this section, the program produces the corresponding differential cascaded spectrum $\left.\frac{\mathrm{d} N_{\gamma}}{\mathrm{d} E_{\gamma}}\right|_{\text {casc }}$ as an output. 
$\gamma$-Cascade uses a semi-analytic approach, taking into account PP, ICS and synchrotron cooling, as well as cosmological expansion effects. The differential spectrum of electrons after the first PP stage in isotropic targets with a spectrum $\frac{\mathrm{d} n_{t}}{\mathrm{~d} \epsilon_{t}}$ is given by (see Eq. 3-34)

$$
\frac{\mathrm{d} N_{e}}{\mathrm{~d} E_{e}}\left(E_{e}\right)=\left.\lambda_{\mathrm{PP}} \iint \frac{\mathrm{d} N_{\gamma}}{\mathrm{d} E_{\gamma}}\right|_{\text {inj }}\left(E_{\gamma}\right) \frac{\mathrm{d} n_{t}}{\mathrm{~d} \epsilon_{t}}\left(\epsilon_{t}\right) \frac{\mathrm{d} \sigma_{\mathrm{PP}}}{\mathrm{d} E_{e}}\left(\epsilon_{t}, E_{\gamma}, E_{e}\right) \mathrm{d} \epsilon_{t} \mathrm{~d} E_{\gamma},
$$

where $\lambda_{\mathrm{PP}}$ is the mean free path traversed by the injected $\gamma$-rays and $\frac{\mathrm{d} \sigma_{\mathrm{PP}}}{\mathrm{d} E_{e}}$ is given by Eq. 3-6.

After one ICS, the electron of energy $E_{e}$ loses energy $\Delta E_{e}$, producing the new $\gamma$-ray spectrum

$$
\frac{\mathrm{d} N_{\gamma^{\prime}}}{\mathrm{d} E_{\gamma^{\prime}}}\left(E_{\gamma^{\prime}}, E_{e}, \Delta E_{e}\right)=\mathcal{N} f\left(E_{e}\right) \lambda_{\mathrm{ICS}} \int \frac{\mathrm{d} n_{t}}{\mathrm{~d} E_{t}}\left(\epsilon_{t}\right) \frac{\mathrm{d} \sigma_{\mathrm{ICS}}}{\mathrm{d} E_{\gamma^{\prime}}}\left(\epsilon_{t}, E_{\gamma^{\prime}}, E_{e}\right) \mathrm{d} \epsilon_{t},
$$

where $\mathcal{N}$ is an overall normalization factor such that energy is conserved, $\Delta E_{e}=\int E_{\gamma^{\prime}} \frac{\mathrm{d} N_{\gamma^{\prime}}}{\mathrm{d} E_{\gamma^{\prime}}} \mathrm{d} E_{\gamma^{\prime}}, f\left(E_{e}\right)$ is the fraction of the injected energy which goes into ICS $\left(f\left(E_{e}\right)<1\right.$ due to synchrotron emission), and $\lambda_{\mathrm{ICS}}$ is the mean free path of electrons before interacting with target photons. The average synchrotron energy loss rate depends on the medium's magnetic field, $B$, the electron's velocity $\beta$ and Lorentz factor, $\gamma_{e}$, as [125]

$$
\frac{\mathrm{d} E_{\mathrm{loss}}}{\mathrm{d} t}=\frac{4}{3} \sigma_{\mathrm{T}} \frac{B^{2}}{8 \pi} \beta^{2} \gamma_{e}^{2} .
$$

To obtain the total cascaded $\gamma$-ray spectrum, one must sum over all spectra from ICS of an electron with energy $E_{e}$, then integrate over all electron energies to consider all ICS events,

$$
\left.\frac{\mathrm{d} N_{\gamma}}{\mathrm{d} E_{\gamma}}\right|_{\text {casc }}\left(E_{\gamma}\right)=\int \frac{\mathrm{d} N_{e}}{\mathrm{~d} E_{e}}\left(E_{e}, E_{\gamma^{\prime}}\right) \sum_{\Delta E_{e}} \frac{\mathrm{d} N_{\gamma^{\prime}}}{\mathrm{d} E_{\gamma^{\prime}}}\left(E_{\gamma^{\prime}}, E_{e}, \Delta E_{e}\right) \mathrm{d} E_{e} .
$$

This integration is done repeatedly over as many propagation steps $n$ necessary such that $n \lambda=D$, where $D$ is the comoving distance from the source to the Earth, assuming a flat universe. Cosmological redshift is also taken into account for both a point source and a diffuse source distribution; see [109] for all the details. 


\subsection{2}

\section{Comparing Simulations with the Universal Spectrum}

As briefly discussed in Section 3.4, the universal spectrum calculated analytically is not valid for sources located at any redshift $z$. In this section, we show some examples of point-source cascaded spectra and evaluate how they compare with Eq. 3-27. Unfortunately, we are only able to analyse the region above $E_{X} \approx 0.1 \mathrm{GeV}$ since this is the lowest energy covered by the $\gamma$ Cascade program. For a comparison including the region below $E_{X}$, see [106].

In our analytical calculation, we consider $\epsilon_{e b l}(z)=\epsilon_{e b l, 0}$ since the COB peak around $\lambda \approx 1 \mu \mathrm{m}$ shifts negligibly up to $z=2$ (see Figure 3.2). The decrease in $E_{t h, e b l}$ by a factor of $(1+z)$ is the only cosmological redshift effect we account for. Although the universal spectrum is independent of the emission, we assume a simple $E^{-2}$ power-law with an exponential cutoff at $10 \mathrm{PeV}$ as the injection spectrum for our simulations.

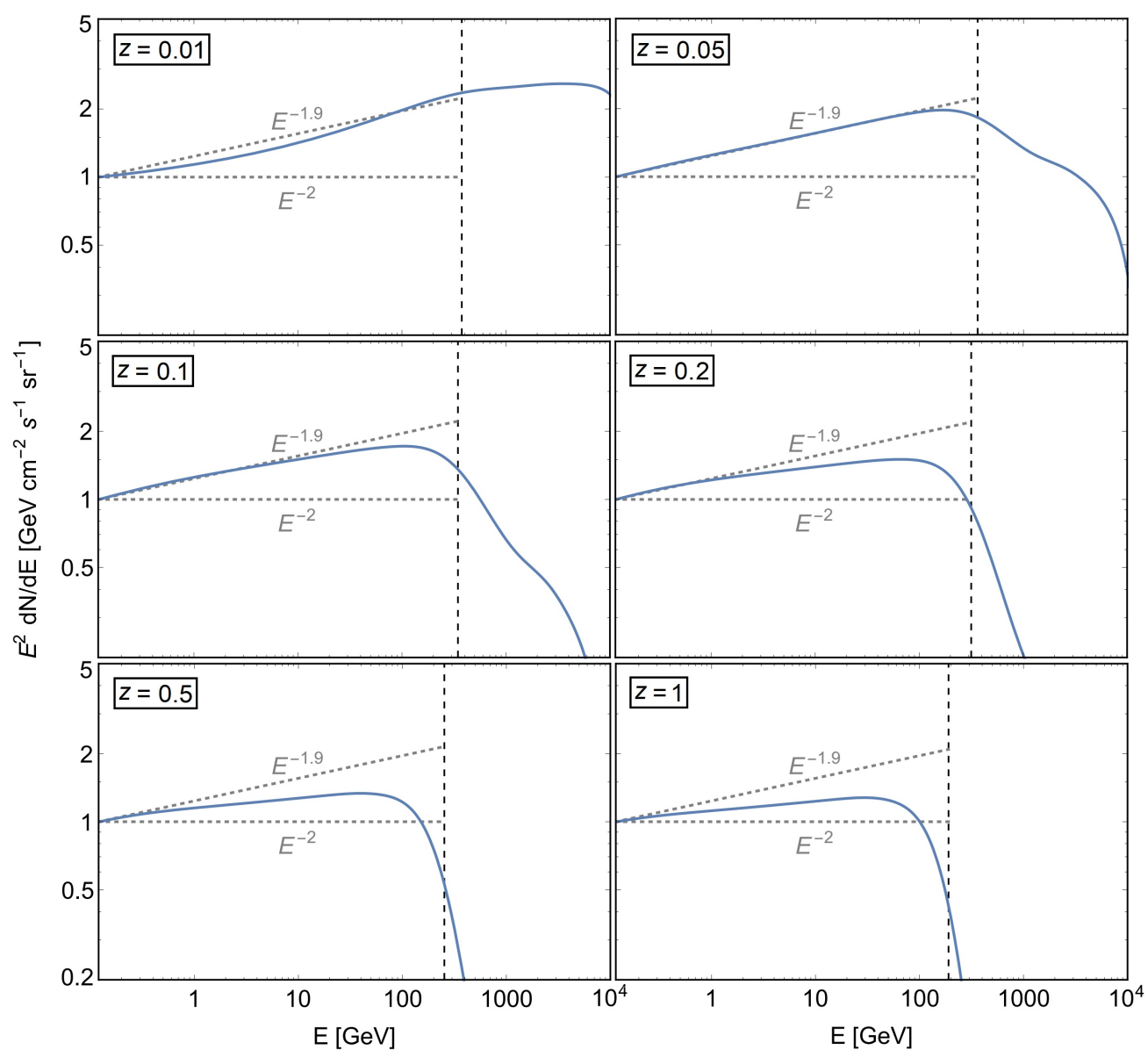

Figure 3.4: Cascaded fluxes (blue lines) above $E_{X} \approx 100 \mathrm{MeV}$ from point sources located at different redshifts. The black dashed line corresponds to the cutoff energy $E_{t h, e b l}(z)$ predicted by the universal spectrum in Eq. 3-27. Dashed grey lines correspond to $E_{-2}$ (as predicted by the Eq. $3-27$ ) and $E^{-1.9}$ spectra. The latter index seems to fit the simulations better for $z \lesssim 0.2$. 
Curiously, our simulations show that the $E_{X} \leq E_{\gamma} \leq E_{t h, e b l}$ part of Eq. 327 is seen as having an approximate $E_{\gamma}^{-1.9}$ dependence instead of the expected $E_{\gamma}^{-2}$. The reason behind this is unclear and should be explored. The smoothness of the cutoff at $E_{t h, e b l}$ is expected due to the EBL not being monochromatic, but its position is relatively accurate (withing a factor of $\sim 3$ ) for all cases except $z=0.01$. This is an exceptional case where the source is too close such that the cascade cannot properly develop, resulting in a cutoff at $\sim 10 \mathrm{PeV}$ instead, where the injection spectrum itself has a cutoff. Simulations including the energy range below $E_{X}$ have shown that the $E^{-1.5}$ low-energy spectrum is robust. 


\section{4}

\section{Methodology}

In Chapter 1, we have presented the evidence coming from IceCube observations for the existence of astrophysical neutrinos, as well as posing the necessity for a multimessenger approach in search for their sources. A natural follow-up would be to look for their counterpart in $\gamma$-rays, which is expected to exist based on the conventional neutrino production scenarios presented in Chapter 2. Chapter 3 has shown us that $\gamma$-rays emitted at $>\mathrm{TeV}$ energies undergo an electromagnetic cascading process, producing an observed $\gamma$-ray flux at the Earth at sub-TeV energies (assuming full development of the cascade). This chapter shows us where we expect to find these $\gamma$-rays and how exactly we can use them to set constraints on the IceCube neutrino sources.

Since IceCube neutrinos have $\sim \mathrm{TeV}-\mathrm{PeV}$ energies, so do their corresponding $\gamma$-rays via the relation in Eq. 2-10. This means that, after they experience the electromagnetic cascade, we are looking for a diffuse $\gamma$-ray flux at sub-TeV energies, which would manifest itself as a contribution to what is known as the extragalactic $\gamma$-ray background (EGB). We shall begin this chapter by presenting the EGB, together with its relevant features and wellestablished contributions. Once that is done, we will be ready to describe the step-by-step methodology of our analysis which will set multimessenger constraints on the sources of astrophysical neutrinos. This includes determining the neutrino injection spectrum by introducing several free parameters, choosing a source candidate (i.e. a source population/distribution of sources), making use of the $\gamma$-Cascade code to calculate the corresponding $\gamma$-ray spectrum at the Earth and defining a $\chi^{2}$-test to quantitatively search for any tensions in the parameter space between IceCube and EGB data.

\section{1}

\section{The Extragalactic $\gamma$-ray Background}

The extragalactic $\gamma$-ray background (EGB), measured between $100 \mathrm{MeV}$ and $820 \mathrm{GeV}$ by the Large Area Telescope (LAT) on board the Fermi Gammaray Space Telescope (Fermi) [126], is the observed flux corresponding to all extragalactic $\gamma$-ray sources, both point-like and diffuse. Figure 4.1 presents the latest measurements reported by the Fermi Collaboration. In this energy 
range, the main challenge in measuring the EGB involves subtracting galactic background contributions. Notably, the galactic center is a very intense and difficult to model source of $\gamma$-rays. For this reason, the Fermi Collaboration only reports EGB intensities for high galactic latitudes $\left(|b|>20^{\circ}\right.$ in galactic coordinates).

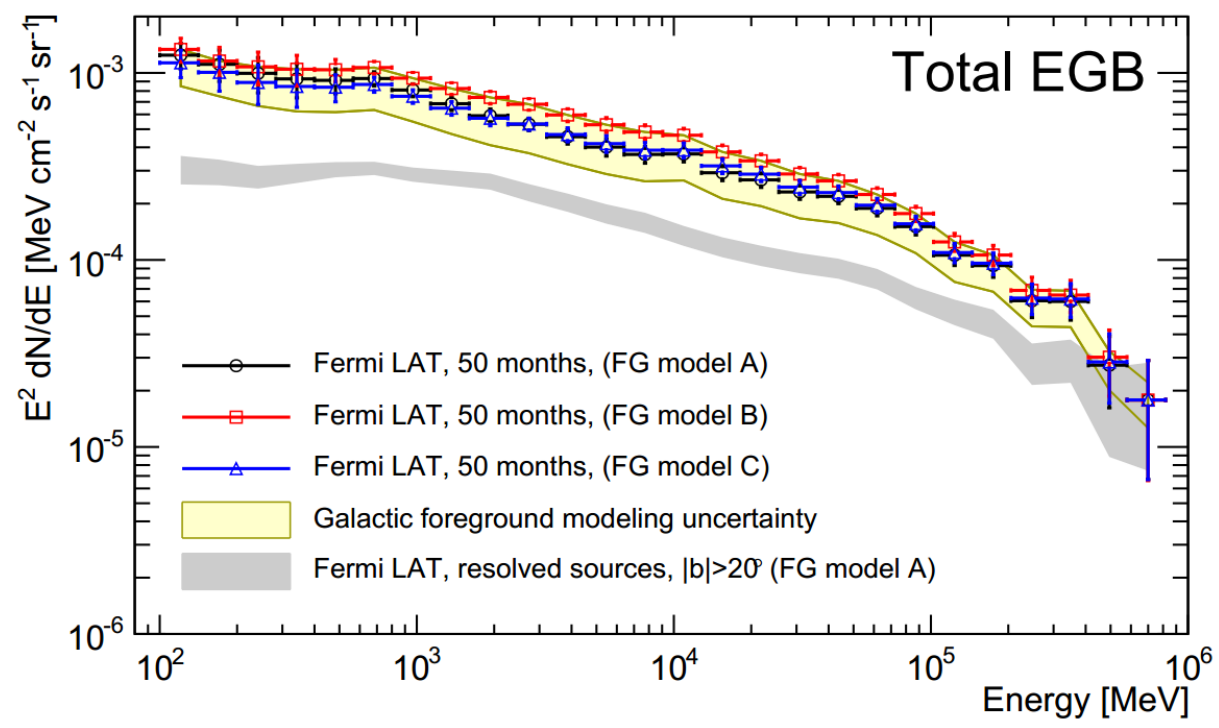

Figure 4.1: EGB flux measured by The Fermi-LAT Collaboration between $100 \mathrm{MeV}$ and $820 \mathrm{GeV}$ for three different foreground models (see Table 4.1) at high latitudes, $|b|>20^{\circ}$ [126]. Error bars include statistical and systematic uncertainties for each foreground model added in quadrature, while the yellow shaded region represents the systematic uncertainty coming specifically from modelling the diffuse galactic emission. For foreground model A, the cumulative intensity solely from resolved sources is shown as the gray band.

Another background which is comparable to the EGB for $\gtrsim 1 \mathrm{GeV}$ energies is the diffuse galactic emission (DGE) resulting from CRs interacting with interstellar gas and radiation fields. To model the DGE, Fermi considered three different assumptions regarding the CR injection and propagation scenarios. They are denoted as foreground (FG) models A, B and C; Table 4.1 briefly outlines their main differences. Each FG model results in different EGB intensities, all displayed in Figure 4.1. Adopting different models does not change qualitatively the results obtained in this thesis and, since it is the most conventional scenario, we choose to use FG model A in our calculations. For clarity, the EGB intensities in each energy bin and the upper uncertainties (statistical, systematic and FG model uncertainty added in quadrature) for FG model A are displayed in Table 4.2. The complete EGB data released by the Fermi Collaboration can be found at [126]. 
Table 4.1: Main differences between the three FG models for the DGE employed in Fermi's EGB analysis [126].

\begin{tabular}{c|c} 
Model A & $\begin{array}{r}\text { Source population injecting CR nuclei and electrons is the same; } \\
\text { constant CR diffusion coefficient throughout the galaxy }\end{array}$ \\
\hline Model B & Addition of an electron source population near the galactic center \\
\hline Model C & $\begin{array}{c}\text { Diffusion coefficient varying with radial distance to galactic center } \\
\text { and height above the galactic plane }\end{array}$
\end{tabular}

Lastly, CRs misclassified as $\gamma$-rays and CR-induced atmospheric $\gamma$-rays constitute a residual background contamination which is not perfectly known. To subtract such mis-reconstructed events, Fermi simulates their presence in the near-Earth environment, obtaining the expected distribution of such events and comparing it with actual data. Achieving the best compromise between a low CR background uncertainty and high statistics is actually the reason why Fermi restricts their analysis between $100 \mathrm{MeV}$ and $820 \mathrm{GeV}$.

Table 4.2: EGB intensities and upper uncertainties, in $\left[\mathrm{cm}^{-2} \mathrm{~s}^{-1} \mathrm{sr}^{-1}\right]$, for FG model A, which is the one used in this work. The uncertainties include the statistical, systematic and foreground modeling errors added in quadrature [126].

\begin{tabular}{|c|c|c|c|}
\hline \multicolumn{2}{|c|}{ Energy Bins $[\mathrm{GeV}]$} & \multirow{2}{*}{$\begin{array}{l}\text { EGB Intensities } \\
{\left[\mathrm{cm}^{-2} \mathrm{~s}^{-1} \mathrm{sr}^{-1}\right]}\end{array}$} & \multirow{2}{*}{$\begin{array}{l}\text { Upper Uncertainties (stat. + syst. + FG.) } \\
\qquad\left[\mathrm{cm}^{-2} \mathrm{~s}^{-1} \mathrm{sr}^{-1}\right]\end{array}$} \\
\hline Lower bound & Upper bound & & \\
\hline 0.1 & 0.1414 & $3.674 \times 10^{-6}$ & $6.331 \times 10^{-7}$ \\
\hline 0.1414 & 0.2 & $2.321 \times 10^{-6}$ & $4.530 \times 10^{-7}$ \\
\hline 0.2 & 0.2828 & $1.469 \times 10^{-6}$ & $3.373 \times 10^{-7}$ \\
\hline 0.2828 & 0.4 & $9.697 \times 10^{-7}$ & $2.364 \times 10^{-7}$ \\
\hline 0.4 & 0.5657 & $6.735 \times 10^{-7}$ & $1.390 \times 10^{-7}$ \\
\hline 0.5657 & 0.8 & $4.871 \times 10^{-7}$ & $8.218 \times 10^{-8}$ \\
\hline 0.8 & 1.1314 & $2.990 \times 10^{-7}$ & $5.267 \times 10^{-8}$ \\
\hline 1.1314 & 1.6 & $1.786 \times 10^{-7}$ & $3.946 \times 10^{-8}$ \\
\hline 1.6 & 2.2627 & $1.089 \times 10^{-7}$ & $2.918 \times 10^{-8}$ \\
\hline 2.2627 & 3.2 & $6.932 \times 10^{-8}$ & $2.009 \times 10^{-8}$ \\
\hline 3.2 & 4.5255 & $4.207 \times 10^{-8}$ & $1.342 \times 10^{-8}$ \\
\hline 4.5255 & 6.4 & $2.618 \times 10^{-8}$ & $8.737 \times 10^{-9}$ \\
\hline 6.4 & 9.051 & $1.692 \times 10^{-8}$ & $5.649 \times 10^{-9}$ \\
\hline 9.051 & 12.8 & $1.203 \times 10^{-8}$ & $3.317 \times 10^{-9}$ \\
\hline 12.8 & 18.1019 & $6.754 \times 10^{-9}$ & $2.074 \times 10^{-9}$ \\
\hline 18.1019 & 25.6 & $4.376 \times 10^{-9}$ & $1.221 \times 10^{-9}$ \\
\hline 25.6 & 36.2039 & $2.668 \times 10^{-9}$ & $7.013 \times 10^{-10}$ \\
\hline 36.2039 & 51.2 & $1.789 \times 10^{-9}$ & $4.052 \times 10^{-10}$ \\
\hline 51.2 & 72.4077 & $1.093 \times 10^{-9}$ & $2.245 \times 10^{-10}$ \\
\hline 72.4077 & 102.4 & $6.183 \times 10^{-10}$ & $1.236 \times 10^{-10}$ \\
\hline 102.4 & 144.8155 & $3.084 \times 10^{-10}$ & $6.608 \times 10^{-11}$ \\
\hline 144.8155 & 204.8 & $1.925 \times 10^{-10}$ & $3.748 \times 10^{-11}$ \\
\hline 204.8 & 289.6309 & $8.880 \times 10^{-11}$ & $2.145 \times 10^{-11}$ \\
\hline 289.6309 & 409.6 & $6.280 \times 10^{-11}$ & $1.605 \times 10^{-11}$ \\
\hline 409.6 & 579.2619 & $2.053 \times 10^{-11}$ & $9.515 \times 10^{-12}$ \\
\hline 579.2619 & 819.2 & $9.663 \times 10^{-12}$ & $6.448 \times 10^{-12}$ \\
\hline
\end{tabular}


Point-like contributions to the EGB at high energies consist almost entirely of blazars, including BL Lacertae objects (BL Lacs) and flat-spectrum radio quasars. In fact, it has been shown in [127] that $86_{-14}^{+16} \%$ of the total EGB above $50 \mathrm{GeV}$ corresponds to contributions from individual sources in the $2 \mathrm{FHL}$ catalog [128], most of them being blazars. Radio galaxies (RGs; also known as misaligned AGN) and star-forming galaxies (SFGs), on the other hand, are mostly too faint to be resolved and become diffuse EGB contributions. In this thesis, we take into account the well-established/conventional contributions from blazars using the luminosity-dependent density evolution model adopted in [129], as well as from RGs [130] and SFGs [131], which alone account for nearly $100 \%$ of the EGB. ${ }^{1}$ They are displayed in Figure 4.2, taken from [129].

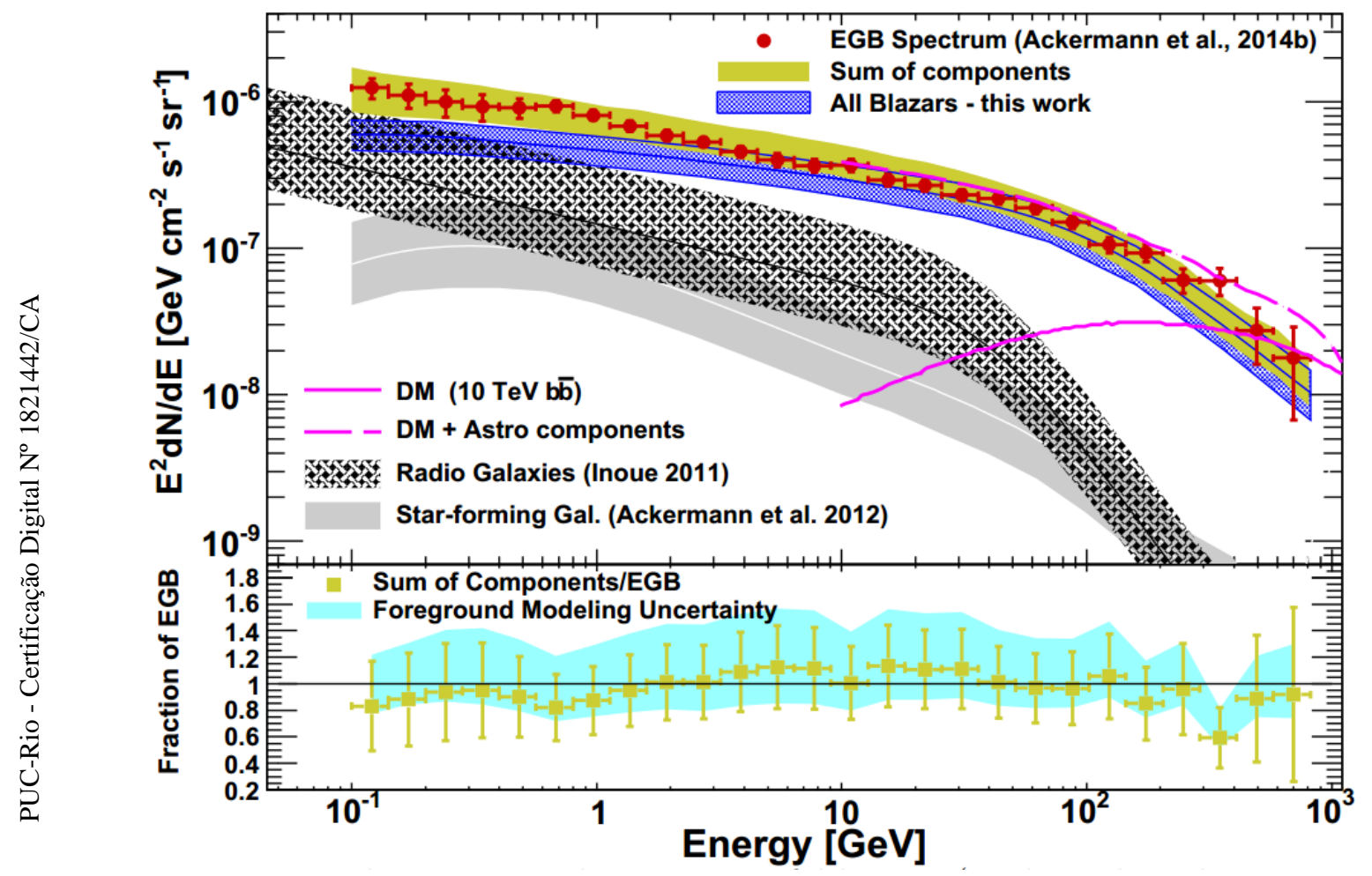

Figure 4.2: EGB data (red points) together with its three conventional contributions considered in this work (blazars: blue band [129], RGs: black striped band [130], SFGs: gray band [131]) and their respective uncertainties. Their sum is represented by the yellow band. Pink lines pertain to the original paper's [129] intention to set constraints on the DM annihilation cross section and are unrelated to this thesis.

\footnotetext{
${ }^{1}$ Other more recent estimates for the blazar, RG and SFG contributions can be found at [132], [133] and [134], respectively. A notable difference is that the new blazar contribution accounts for $\approx 100 \%$ of the EGB intensity at energies $\gtrsim 10 \mathrm{GeV}$, while RGs dominate at $\approx 0.1-1 \mathrm{GeV}$. Nevertheless, these differences will not change significantly the results obtained in this thesis.
} 
If we remove all individually resolved sources (together with all known galactic foregrounds and mis-reconstructed CR events) from the EGB, the leftover $\gamma$-ray flux is known as the isotropic diffuse $\gamma$-ray background (IGRB), displayed in Figure 4.3(a) for all three FG models. Multi-wavelength observations have identified most of the contributions to the IGRB, including the aforementioned RG (i.e. "non-blazar AGN") and SFG contributions, as well as a smaller fraction $(\lesssim 20 \%)$ coming from blazars [135, 136, 137, 138] and merging galaxy clusters $[139,140]$. Leading contributions are shown in Figure 4.3(b), with their corresponding error bands; they already provide a good fit for the entire IGRB by themselves and adding any extra contributions runs the risk of overshooting the data. Notwithstanding, there is still very narrow margin for unknown/yet-to-be-considered extragalactic sources and unresolved galactic emissions. Examples of unknown extragalactic source candidates include the IceCube neutrino sources (which should produce cascaded $\gamma$-rays at energies within the EGB range) as well as $\gamma$-rays originating from BSM processes such as DM annihilation [129] or decay [141]. As for galactic contributions to the IGRB, CR interactions with gas and radiation fields in the galactic halo, with solar system debris and with solar radiation have all been considered [142, 143, 144, 145, 146, 147]. However, the leading galactic contribution is expected to be from a hidden population of millisecond pulsars, accounting for $\lesssim 0.9 \%$ of the EGB [148].

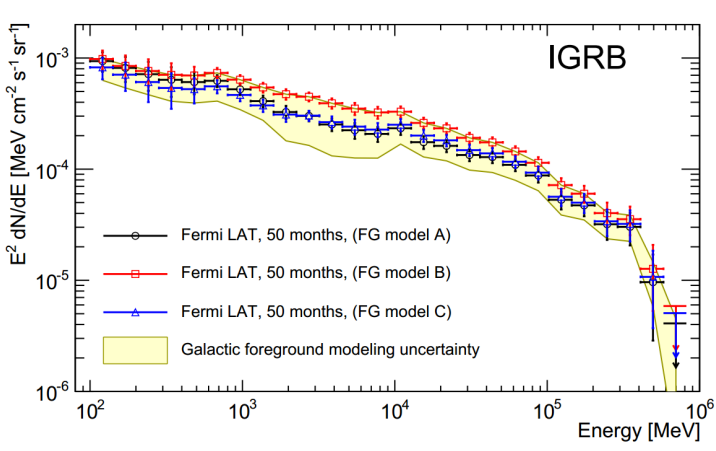

(4.3(a)) IGRB

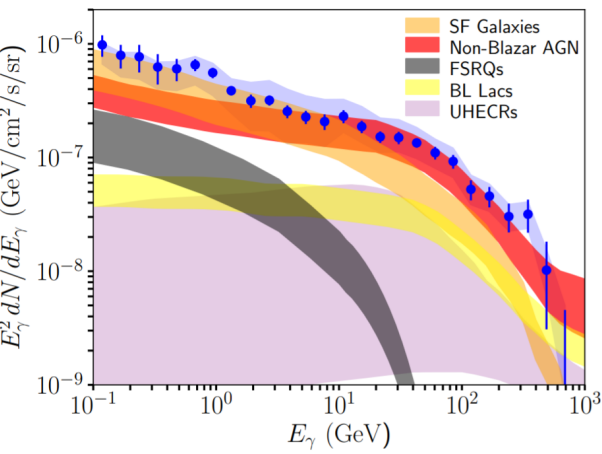

(4.3(b)) IGRB Contributions

Figure 4.3: Left: same as Figure 4.1 but for the IGRB [126]. Right: Conventional/well-established contributions to the IGRB, together with their sum and total uncertainty represented by the blue band [141].

Needless to say, the EGB is an essential feature for our multimessenger analysis. It is where we expect to find the $\gamma$-ray counterpart of IceCube's astrophysical neutrinos. In the following section, we will describe how we can use the EGB to establish constraints on the possible neutrino sources. 


\section{2 \\ Multimessenger Analyses}

We have just seen that the EGB is comprised of conventional sources, of which we will consider blazars, RGs and SFGs, and unknown/unidentified diffuse sources which end up in the IGRB. Searches for new physics contributions to the IGRB such as from DM decay [141] have already been done in the past. Here, we will instead consider a contribution which is naturally predicted by the Standard Model: the $\gamma$-ray counterpart of the IceCube astrophysical neutrino flux (henceforth referred to as the "cascaded" $\gamma$-ray contribution). Because we expect such a contribution to exist, provided that their sources are $\gamma$-ray transparent, this study can set robust multimessenger constraints on the astrophysical neutrino source candidates.

However, one should note that the IGRB is not a fundamental quantity. In contrast to the EGB, it is highly detector-dependent: a more precise detector would resolve more individual sources and have less background contamination, thus measuring a smaller IGRB intensity. The EGB, however, stays the same. Naively imposing that the cascaded $\gamma$-ray contribution must not exceed the IGRB, as was done in [141], sets constraints that eventually become obsolete once the EGB is measured with better precision. Also, this approach fails to account for the uncertainties in the known point-like contributions to the EGB, represented by the shaded bands in Figure 4.2. We are forced to conclude that a statistically meaningful analysis must incorporate the EGB instead.

With this in mind, we require that the sum of the cascaded and conventional contributions does not exceed the total EGB intensity. To implement this condition rigorously, we will define a $\chi^{2}$ function which correctly takes into account the conventional contributions' uncertainties. This is the main analysis of this thesis, and will be used to constrain possible emission scenarios for a given distribution of IceCube neutrino sources. To check our results, we perform a complementary analysis in Section 4.2.2 based on the integrated EGB flux above $50 \mathrm{GeV}$.

\subsection{1}

$\chi^{2}$ Test

For reasons presented in Chapter 2, we expect that the injected astrophysical neutrino spectrum takes on a broken power-law shape, such as the one in Eq. 2-6. In order to obtain more robust results, we wish to be as conservative as possible in our assumptions. Hence, we adopt the following minimal assumption: 


$$
\frac{\mathrm{d} N_{\nu}}{\mathrm{d} \varepsilon_{\nu}}\left(\varepsilon_{\nu}\right)= \begin{cases}B & \varepsilon_{\nu}<\varepsilon_{\mathrm{br}} \\ A \varepsilon_{\nu}^{-s_{h}} & \varepsilon_{\mathrm{br}}<\varepsilon_{\nu}<\varepsilon_{\mathrm{cut}} \\ 0 & \varepsilon_{\nu}>\varepsilon_{\mathrm{cut}}\end{cases}
$$

where $A$ and $B$ are normalization constants satisfying $B=A \varepsilon_{\mathrm{br}}^{-s_{h}}$ for continuity at $\varepsilon_{\nu}=\varepsilon_{\mathrm{br}}$. We impose a sharp cutoff at $\varepsilon_{\text {cut }}=10 \mathrm{PeV}$ because IceCube has not seen any neutrinos with higher energy. However, varying $\varepsilon_{\text {cut }}$ between 1$100 \mathrm{PeV}$ has a negligible effect on the corresponding cascaded $\gamma$-ray flux. The main contribution to the cascaded flux which adds to the EGB comes from the low-energy part of Eq. 4-1, characterized by $\varepsilon_{\text {br }}$. Note that Eq. 4-1 can be realised in the $p \gamma$ scenario $\left(s_{l}=0\right.$ in Eq. 2-6). Although this is something to keep in mind as a likely production mechanism of IceCube's astrophysical neutrinos, the main reason for adopting the spectrum in Eq. 4-1 is simply to make our analysis as conservative as possible.

At the end of the day, we are left with 3 free parameters, $A, \varepsilon_{\mathrm{br}}$ and $s_{h}$, which will be scanned over. Each of them is directly related to a measured quantity in IceCube's flux from Eq. 2-1:

$$
\begin{aligned}
A & \propto \Phi_{\text {astro }} \\
s_{h} & =\gamma_{\text {astro }} \\
\varepsilon_{\mathrm{br}} & \lesssim E_{\mathrm{th}} .
\end{aligned}
$$

The precise relations between $A$ and $\Phi_{\text {astro }}$, and $\varepsilon_{\text {br }}$ and $E_{\text {th }}$ are the subject of Sections 4.2 .3 and 4.2.4, respectively, but we can already see from $s_{h}=\gamma_{\text {astro }}$ that IceCube's fit in Eq. 2-1 corresponds to the region $\varepsilon_{\text {br }}<\varepsilon_{\nu}<\varepsilon_{\text {cut }}$ in Eq. 4-1. From now on, we shall use $s_{h}$ and $\gamma_{\text {astro interchangeably. }}$

Given a choice of $A, \varepsilon_{\mathrm{br}}$ and $s_{h}$, we obtain the corresponding $\gamma$-ray injection spectrum via relation 2-10 and supply it to $\gamma$-Cascade, together with a specific source density distribution (see Appendix B). The program then calculates the cascaded $\gamma$-ray flux at the Earth coming from this source population, producing a diffuse contribution to the EGB. We are now ready to elucidate our method for setting constraints on the $\left(A, \varepsilon_{\mathrm{br}}, s_{h}\right)$ parameter space.

This is achieved by defining a $\chi^{2}$ test statistic given by

$$
\chi^{2}=\min _{\mathcal{A}}\left[\sum_{i} \frac{\left(F_{i, \mathrm{EGB}}-\mathcal{A} F_{i \text {,astro }}-F_{i, \mathrm{cas}}\right)^{2}}{\sigma_{i}^{2}}+\frac{(\mathcal{A}-1)^{2}}{\sigma_{\mathcal{A}}^{2}}\right],
$$

where the index $i$ runs over all 26 Fermi energy bins. $F_{i \text {,EGB }}$ and $\sigma_{i}$ are the EGB intensity and uncertainty, respectively, in the $i$-th bin. $F_{i \text {,astro }}$ corresponds to the sum of all known astrophysical components (blazars + RGs + SFGs) to the 
EGB and $F_{i \text {,cas }}$ is the cascaded $\gamma$-ray contribution to the $i$-th energy bin. $F_{i \text {,astro }}$ is allowed to vary in normalization within the yellow shaded region in Figure 4.2 through the introduction of a nuisance parameter, $\mathcal{A}$. To compensate for this freedom of normalization which allows for a better fit to the EGB, one should include the Gaussian-type pull term $\left[(\mathcal{A}-1) / \sigma_{\mathcal{A}}\right]^{2}{ }^{2}$ Here, $\sigma_{\mathcal{A}} \approx 35 \%$ is the average normalization uncertainty of the conventional astrophysical contributions over all bins.

The allowed region in an $m$-parameter space (e.g. $m=2$ free parameters such as $\varepsilon_{\text {br }}$ and $s_{h}$, while keeping $A$ fixed) is obtained by requiring that $\Delta \chi^{2}=\chi^{2}-\chi_{\min }^{2}<q$; the values of $q$ are reported in Table 4.3 for several confidence levels (CL). Here, $\chi_{\min }^{2} \approx 27.03$ is the smallest $\chi^{2}$ found from Eq. 4-5 when scanning over the entire $\left(A, \varepsilon_{\mathrm{br}}, s_{h}, \mathcal{A}\right)$ parameter space, conveniently located at $\mathcal{A} \approx 1$ and $A=0$.

Table 4.3: Values of $q$ such that $\Delta \chi^{2}>q$ implies an exclusion of a scenario at a specific confidence level (CL). The values are reported for $m=1,2$ and 3 free parameters. This table is based on Table 40.2 from Particle Data Group's 2020 Statistics review [149].

\begin{tabular}{c|c|c|c} 
CL $(\%)$ & $m=1$ & $m=2$ & $m=3$ \\
\hline $68.27(1 \sigma)$ & 1.00 & 2.30 & 3.53 \\
90 & 2.71 & 4.61 & 6.25 \\
95 & 3.84 & 5.99 & 7.82 \\
$95.45(2 \sigma)$ & 4.00 & 6.18 & 8.03 \\
99 & 6.63 & 9.21 & 11.34 \\
$99.73(3 \sigma)$ & 9.00 & 11.83 & 14.16
\end{tabular}

The $\chi^{2}$ expression 4-5 has been used before in a similar context by the Fermi Collaboration [129], setting constraints on DM properties simply by swapping $F_{i \text {,cas }}$ by the $\gamma$-ray contribution arising from DM annihilation scenarios. In this work we are not searching for new physics, but instead for a component which is already predicted by the Standard Model and should be present if the IceCube neutrino sources are $\gamma$-ray transparent. Needless to say, this analysis method is adequate for the task; any inconsistencies would impact on several well established, generally accepted and widely used DM limits.

\footnotetext{
${ }^{2}$ Using a $\log$-normal pull-term $\left[\ln (\mathcal{A}) / \ln \left(1+\sigma_{\mathcal{A}}\right)\right]^{2}$ which accounts symmetrically for upper and lower uncertainties does not change significantly the results from this thesis.
} 


\subsection{2}

\section{Integrated Flux above $50 \mathrm{GeV}$}

As a complementary analysis, we recall an important result mentioned in Section 4.1: “... 86 $86_{-14}^{+16 \%}$ of the total EGB above $50 \mathrm{GeV}$ corresponds to contributions from individual sources in the 2FHL catalog, most of them being blazars." If we integrate the EGB spectrum $\Phi_{\mathrm{EGB}}$ above $50 \mathrm{GeV}$, we obtain

$$
F_{\mathrm{EGB}}^{>50 \mathrm{GeV}}=\int_{50 \mathrm{GeV}}^{820 \mathrm{GeV}} \mathrm{d} E_{\gamma} \Phi_{\mathrm{EGB}}\left(E_{\gamma}\right) \simeq 2.4 \times 10^{-9} \mathrm{ph} / \mathrm{cm}^{2} / \mathrm{s} / \mathrm{sr} .
$$

Thus, by requiring

$$
F_{\text {cas }}^{>50 \mathrm{GeV}}=\int_{50 \mathrm{GeV}}^{820 \mathrm{GeV}} \mathrm{d} E_{\gamma} \Phi_{\mathrm{cas}}\left(E_{\gamma}\right)<(1-q) F_{\mathrm{EGB}}^{>50 \mathrm{GeV}},
$$

where $q$ is the percentage of EGB above $50 \mathrm{GeV}$ corresponding to blazars (with mean value of $86 \%)$, we can also set constraints on the $\left(A, \varepsilon_{\mathrm{br}}, s_{h}\right)$ parameter space. Clearly, the choice of parameters affects the resulting cascaded $\gamma$-ray spectrum, $\Phi_{\text {cas }}$.

This analysis has the disadvantage that it is not sensitive to the spectral shape, only to its integral above $50 \mathrm{GeV}$. An excess at any energy bin can be compensated by a deficit at other bins, hiding any tension arising from overshooting and undershooting the EGB data. Indeed, our first/main analysis method would show a tension in this case. We can still use this as a sanity check of our main results, which should produce bounds not too different from the ones obtained via Eq. 4-7.

\subsection{3}

\section{Spectrum Normalization}

In this section, we shall describe exactly how we calculate the normalization of our neutrino injection spectrum for a given fit to the IceCube data. This normalization is given by the constant $A$ in Eq. 4-1, which we already mentioned is proportional to $\Phi_{\text {astro }}$, the IceCube fit normalization. Recall that each of the IceCube datasets (HESE, Cascade and TG, see Section 2.1.2) employs a single power-law fit:

$$
\Phi_{\nu}^{\mathrm{IC}}\left(E_{\nu}\right)=\Phi_{\text {astro }} \times 10^{-18}\left(\frac{E_{\nu}}{100 \mathrm{TeV}}\right)^{-\gamma_{\text {astro }}},
$$

with best-fit values summarized in Table 2.1. This measured flux corresponds to the flux we obtain after propagating the injected spectrum in Eq. 4-1 from their sources to the Earth. More specifically, we are interested in the energy range between $\varepsilon_{\mathrm{br}}<\varepsilon_{\nu}<\varepsilon_{\mathrm{cut}}$, since $\varepsilon_{\mathrm{br}}$ corresponds to $E_{\mathrm{th}}$ which is the lowest 
energy that IceCube considers in their fit. By means of Eq. 2-13, we find this flux to be

$$
\Phi_{\nu}^{\text {diff }}\left(E_{\nu}\right)=\frac{c}{4 \pi} \int_{0}^{\infty} \mathrm{d} z \frac{\mathcal{F}(z)}{H(z)} A(1+z)^{-s_{h}} E_{\nu}^{-s_{h}} .
$$

To find our normalization constant $A$, we equate 4-8 and 4-9 with $\gamma_{\text {astro }}=s_{h}$, solving for $A$ :

$$
A=\Phi_{\text {astro }}\left(\frac{1}{100 \mathrm{TeV}}\right)^{-s_{h}}\left[\frac{c}{4 \pi} \int_{0}^{\infty} \mathrm{d} z \frac{\mathcal{F}(z)}{H(z)}(1+z)^{-s_{h}}\right]^{-1} .
$$

This means that our injection spectrum normalization $A$ depends not only on $\Phi_{\text {astro }}$ but also on $s_{h}{ }^{3}$ Note that we are not sensitive to the overall normalization of $\mathcal{F}(z)$ : $A$ is inversely proportional to it, but this will later be compensated when we evaluate the integral 4-9 or simulate the $\gamma$-ray cascade, which also depends linearly on the normalization of $\mathcal{F}(z)$.

\subsection{4}

\section{$\varepsilon_{\text {br }}$ Redshift}

Now that we know how to obtain $\Phi_{\text {astro }}$ and $s_{h}$, what remains is only $\varepsilon_{\text {br }}$. Since we are working with a minimal assumption, we will assume that there is a cutoff in the observed astrophysical neutrino flux below $E_{\text {th }}$ for each dataset. In the injection spectrum, this is represented by the $\varepsilon_{\nu}<\varepsilon_{\mathrm{br}}$ region, where the power-law index is $s_{l}=0$.

For a point source at fixed redshift $z$, the break energy at the Earth is given by $E_{\mathrm{br}}=\varepsilon_{\mathrm{br}} /(1+z)$. However, for a distribution of sources $\mathcal{F}(z)$, this relation becomes nontrivial. For example, the position of $E_{\mathrm{br}}$ becomes $s_{h}$-dependent and the sharp break is smoothed out due to sources located at different redshifts. What is done in this case is the following: for a 2-dimensional grid of $\varepsilon_{\mathrm{br}}$ and $s_{h}$ values, we calculate the observed flux at the Earth via Eq. 2-13 and find its maximum, which occurs at $E_{\mathrm{br}}$. After interpolation, we end up with a function relating $E_{\mathrm{br}}$ and $\varepsilon_{\mathrm{br}}$ (which also depends on $s_{h}$ ), allowing us to easily find $\varepsilon_{\mathrm{br}}$ corresponding to $E_{\mathrm{br}}=E_{\mathrm{th}}$. The result is shown in Figure 4.4 for different $s_{h}$ values. We can clearly see that a larger $s_{h}$ causes $E_{\mathrm{br}}$ to be smaller with respect to $\varepsilon_{\mathrm{br}}$. This difference increases with the increase of $\varepsilon_{\mathrm{br}}$, reaching $E_{\mathrm{br}} \approx \varepsilon_{\mathrm{br}} / 2$ in the extreme case of $s_{h}=3.4$ and $\varepsilon_{\mathrm{br}}=200 \mathrm{TeV}$.

${ }^{3}$ It does not, however, depend on $\varepsilon_{\text {br }}$ due to our convenient normalization convention in Eq. 4-1. This greatly reduced the computational efforts to probe the full parameter space. 


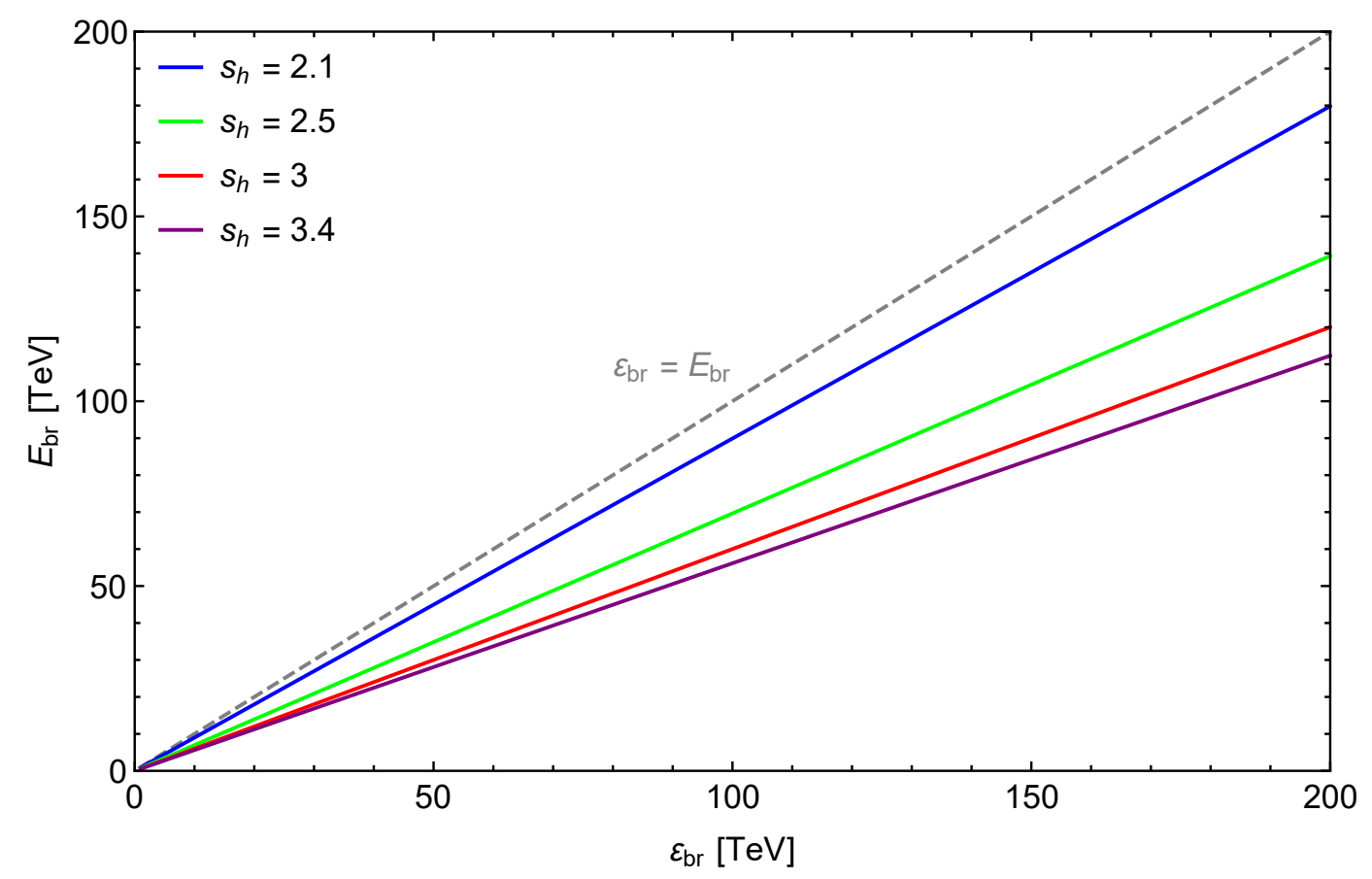

Figure 4.4: Cosmological redshift of the break energy in our injection spectrum 4-1. As expected, this effect results in $E_{\mathrm{br}}<\varepsilon_{\mathrm{br}}$ since all colored lines fall below the dashed gray line (which indicates no redshift).

A similar correction must be done for the cutoff energy $\varepsilon_{\text {cut }}$, which should lead to $E_{\text {cut }}=10 \mathrm{PeV}$ at the Earth. Although this is rigorously required, our results are almost unaffected by changing $E_{\text {cut }}$, as discussed in Section 4.2.1. 


\section{5}

\section{Results and Discussions}

In this chapter, we will present all the results obtained in this thesis, which can also be found in [79]. First, our main $\chi^{2}$ analysis results are shown in Section 5.1, followed by our complementary analysis results in Section 5.2. Evidence of a strong tension (spoiler alert!) between IceCube and EGB data will lead us to many interesting discussions regarding the possible sources of astrophysical neutrinos. Section 5.3 contemplates some of the most common solutions currently being studied to alleviate our newly-quantified multimessenger tension. Although our results are only valid for a source density distribution resembling the star formation rate (see Appendix B), a thorough and complete consideration of other distributions has been made in a follow-up paper also co-authored by me [80], confirming the robustness of our results.

\section{1}

\section{$\chi^{2}$ Analysis Results}

Based on the methodology described in Section 4.2.1, we present 2dimensional projections of our 3-dimensional parameter space $\left(A, \varepsilon_{\mathrm{br}}, s_{h}\right)$ (or equivalently, $\left.\left(\Phi_{\text {astro }}, E_{\mathrm{br}}, \gamma_{\text {astro }}\right)\right)$. These include limits in the $\varepsilon_{\mathrm{br}}-s_{h}$ plane for fixed normalization values and limits in the $\Phi_{\text {astro- }} \gamma_{\text {astro }}$ plane for fixed $E_{\mathrm{br}}$ values. The former will reveal to us a clear tension between IceCube and EGB data, which is then quantified in the latter.

\section{1 .1}

\section{Limits in the $\varepsilon_{\mathrm{br}}-s_{h}$ Plane}

Let us first recall the $1 \sigma$ contours in the $\Phi_{\text {astro- }} \gamma_{\text {astro }}$ plane reported by IceCube in their 7.5y HESE, 6y Cascades and 9.5y Tracks datasets, displayed in Figure 2.7. Upon fixing $\gamma_{\text {astro }}$ (i.e. limiting oneself to a vertical line passing through $\gamma_{\text {astro }}$ ), one can easily find the highest and lowest allowed normalizations for a given dataset, which we will call $\Phi_{\max }$ and $\Phi_{\min }$, respectively. For instance, the 7.5y HESE data allows for $\Phi_{\min }=1.66<\Phi_{\text {astro }}<2.91=\Phi_{\max }$ at $1 \sigma$ CL when $\gamma_{\text {astro }}=3$.

In this first analysis, we perform a scan over $\varepsilon_{\text {br }}$ from $1-200 \mathrm{TeV}$ and $s_{h} \in[2.15,3.2]$, which includes the $1 \sigma s_{h}$ ranges for all three datasets. For 
each $s_{h}$ value, we normalized our injection spectra with both $A_{\max }$ and $A_{\min }$, corresponding to $\Phi_{\max }$ and $\Phi_{\min }$ via relation $4-10 .{ }^{1}$ After following our standard routine of simulating the corresponding $\gamma$-ray cascade for each $\left(A, \varepsilon_{\mathrm{br}}, s_{h}\right)$ grid point and calculating their respective $\chi^{2}$ values by comparing with EGB data, we can derive a $90 \%$ CL contour $\left(\Delta \chi^{2}=4.61\right.$ for 2 d.o.f. $)$ in the $\varepsilon_{\mathrm{br}}-s_{h}$ plane separating the allowed and excluded regions. Figure 5.1 shows these limits in blue, green and orange for the 9.5y Tracks, 6y Cascades and 7.5y HESE datasets respectively, with arrows pointing towards the allowed regions and forbidden regions being color-shaded. Upper(lower) contours are for $A_{\max }\left(A_{\min }\right)$, henceforth referred to as "high(low) norm contours". As expected, a higher normalization yields stronger constraints. The real exclusion is probably located somewhere between the high and low norm contours for each dataset. The oval-like shape of these regions is mostly determined by the shape of the contours in Figure 2.7: at the $s_{h}$-extremes of each dataset, where $\Phi_{\max }$ and $\Phi_{\min }$ meet, the high and low contours also meet in Figure 5.1, while at the center of each dataset's $s_{h}$-range, $\Phi_{\max }$ and $\Phi_{\min }$ are further apart, resulting in distant high and low norm contours.

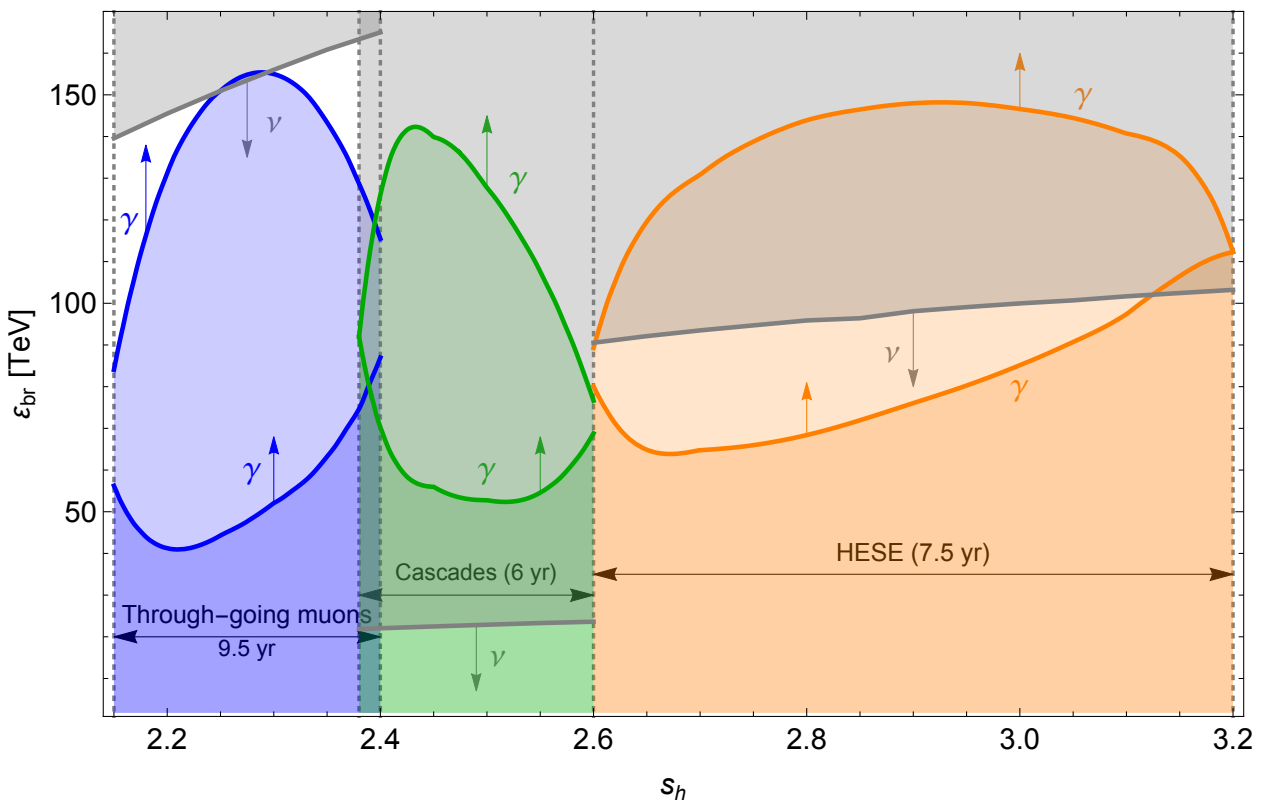

Figure 5.1: Limits in the $\varepsilon_{\mathrm{br}}-s_{h}$ plane from IceCube (gray contours, corresponding to $\left.E_{\mathrm{br}}=E_{\mathrm{th}}\right)$ and EGB data (colored contours, at $90 \%$ CL corresponding to $\left.\Delta \chi^{2}=4.61\right)$, with arrows pointing towards the allowed regions. EGB constraints are obtained assuming the smallest (lower contours) and largest (upper contours) normalizations allowed by IceCube at $1 \sigma$ for each $s_{h}$, according to Figure 2.7, for each dataset (9.5y Tracks: blue, 6y Cascades: green, 7.5y HESE: orange). A clear tension can be seen between neutrino and $\gamma$-ray data in the Cascade $s_{h}$ range. Figure reproduced from [79].

\footnotetext{
${ }^{1}$ Note that, for $s_{h}$ values in the intersection of two datasets, each dataset has its own $1 \sigma$ limits $A_{\min }<A<A_{\max }$.
} 
So far, we have only restricted the parameter space where IceCube is compatible with EGB data. However, we know that $E_{\mathrm{br}} \leq E_{\text {th }}$ (for each dataset) because IceCube does not see any change in spectral index down to $E_{\mathrm{th}} . E_{\mathrm{br}}$ is therefore expected to occur at lower energies, where atmospheric backgrounds limit IceCube's sensitivity. We can translate this restriction on $E_{\mathrm{br}}$ to a restriction on $\varepsilon_{\mathrm{br}}$ using the method described in Section 4.2.4. This is represented in Figure 5.1 by the gray contours, where once again the arrow indicates allowed and shaded indicates excluded regions.

While the through-going muon and HESE datasets are still compatible with the $\operatorname{EGB}$ ( $\gamma$ and $\nu$ contours do not exclude the whole parameter space), the Cascade data, on the other hand, clearly indicates a tension between neutrino and $\gamma$-ray constraints. This is a consequence of the low $E_{\mathrm{th}}$, lowering the gray $\nu$ contour such that even the low norm $\gamma$ contour is excluded by quite a wide margin. At this point, it is important to recognize two facts:

( $i$ ) Even though IceCube separates its neutrino events into different datasets, one should always keep in mind that the astrophysical neutrino flux we are observing in each dataset is the same "one" flux. In other words, a tension in the Cascade dataset cannot be ignored even if the other datasets are allowed by the EGB. We are truly observing astrophysical neutrinos down to $E_{\mathrm{th}}=16$ $\mathrm{TeV}$, and if we extend this threshold into the $s_{h}$ range of the other datasets, this tension would still exist (and even increase in the HESE $s_{h}$ range).

(ii) The $E_{\text {th }}=16 \mathrm{TeV}$ threshold reported by IceCube is very conservative. There are indeed astrophysical neutrino events at IceCube with lower energies $(\sim 1 \mathrm{TeV})$, but they are rejected in the fits to avoid too much background contamination. Lowering the gray contours would certainly increase the tension in Figure 5.1.

It is interesting to see where exactly in EGB energy range are the colored exclusions in Figure 5.1 coming from. In other words, which Fermi energy bins are being overshot by the conventional plus cascaded $\gamma$-ray flux for a given choice of $\left(A, \varepsilon_{\mathrm{br}}, s_{h}\right)$ ? Figure 5.2 shows the neutrino, cascaded and total $\gamma$-ray flux for several $\left(\varepsilon_{\mathrm{br}}, s_{h}\right)$ values with normalizations ranging from $\Phi_{\text {min }}$ to $\Phi_{\max }$ for each IceCube dataset. Also displayed are $\mathcal{A}$ obtained from the $\chi^{2}$ minimization in each case, both for $\Phi_{\min }$ and $\Phi_{\max }(\mathcal{A} \in[0.1,5]$ in our analysis). The dashed lines represent the allowed range based on IceCube's $1 \sigma$ limits on $\Phi_{\text {astro }}$ and $\gamma_{\text {astro }}$, extending down to $E_{\text {th }}$. 


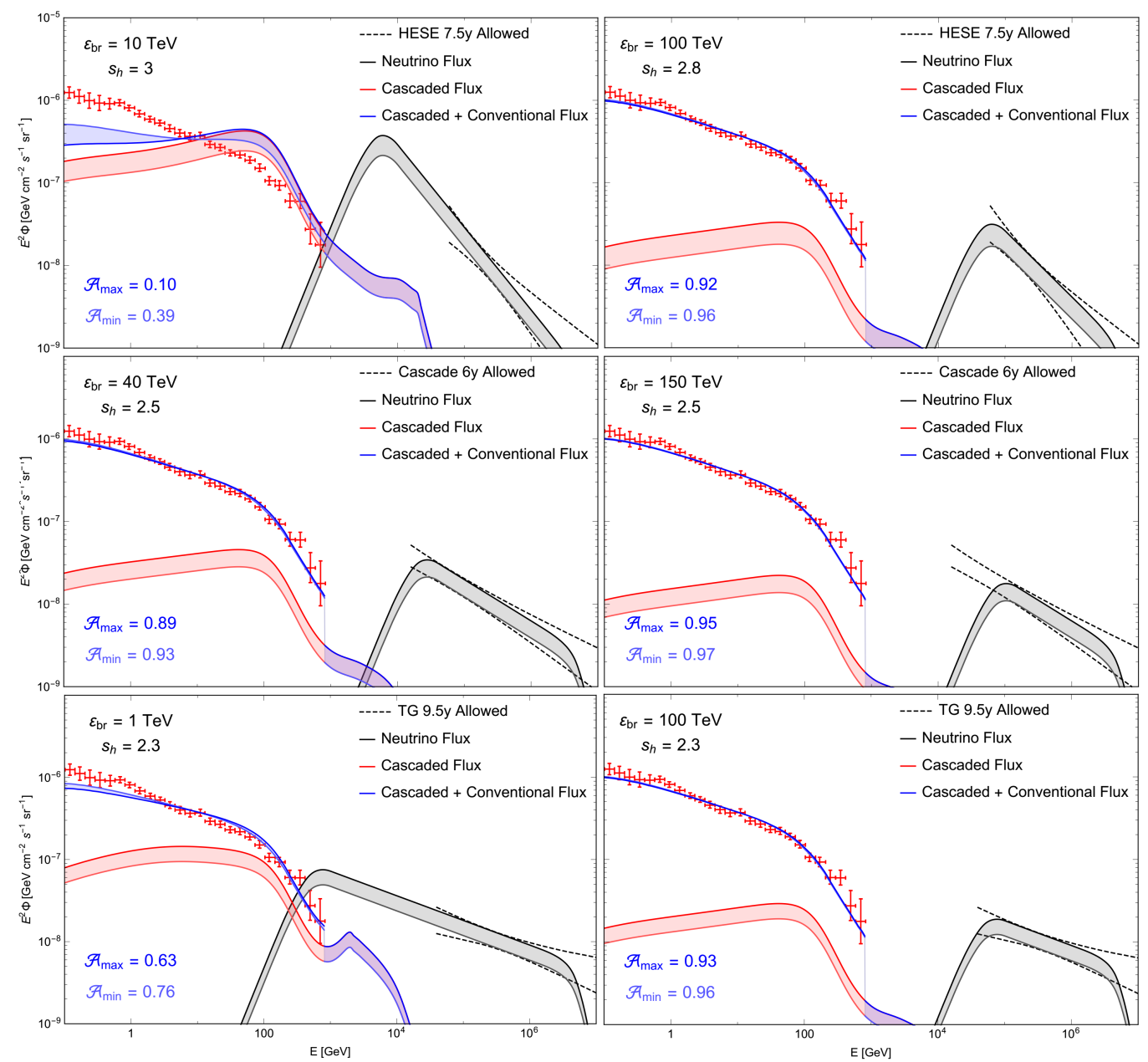

Figure 5.2: Neutrino (black lines), cascaded (red lines) and total (conventional + cascaded, blue lines) $\gamma$-ray fluxes for specific $\left(\varepsilon_{\mathrm{br}}, s_{h}\right)$ values, compared with the IceCube allowed regions for each dataset (black dashed lines) and the Fermi EGB data (red data points). For each flux, the darker tone has normalization $\Phi_{\max }$ while the lighter tone has normalization $\Phi_{\min }$, with a filling of the same color between both lines. $\mathcal{A}_{\text {min }}$ and $\mathcal{A}_{\text {max }}$ are the $\mathcal{A}$ values obtained from the minimization of our $\chi^{2}$ function $4-5$.

It is useful to look at the points in Figure 5.1 corresponding to the plots in Figure 5.2. In most excluded cases, the cascade contribution overshoots the EGB data points around $\sim 10-200 \mathrm{GeV}$, with the notable exception of the upper left plot, where the low $\varepsilon_{\text {br }}$ combined with the large spectral index extends this tension up to $\sim 500 \mathrm{GeV}$. This particular case has the smallest $\mathcal{A}$ values as the $\chi^{2}$ minimization does its best to reduce the conventional contribution and lower the tension, resulting in a peculiar overlap between the red and blue curves, and an inversion of the high and low normalization blue curves. Figure 5.2 also clearly shows the excluded cases where $E_{\mathrm{br}}>E_{\mathrm{thr}}$ where the black neutrino curves cross the dashed lines. The $\lesssim 10 \mathrm{GeV}$ region of the EGB is never a source of tension since the cascaded fluxes do not contribute significantly in that energy range. 
Alas, we are indeed faced with a multimessenger tension which we must quantify in order to determine its significance. From Figure 5.1, we can say that it is certainly larger than $1 \sigma$ (since we are considering the $1 \sigma$ deviations in $\Phi_{\text {astro }}$ and $\left.\gamma_{\text {astro }}\right)$, but perhaps we may have a $\sim 2-3 \sigma$ tension in our hands. The results form the following section will help us determine this significance with more precision.

\section{1 .2}

\section{Limits in the $\Phi_{\text {astro }}-\gamma_{\text {astro }}$ Plane}

With our $\varepsilon_{\text {br- }}-s_{h}$ grid already scanned over with $\Phi_{\text {astro }}=1$, we can vary over several normalizations simply by multiplying the cascaded spectra by the desired $\Phi_{\text {astro. }}{ }^{2}$ In this second analysis, we perform an additional scan over $\Phi_{\text {astro }} \in\left[10^{-2.5}, 10\right]$ in logarithmic steps, calculating the $\chi^{2}$ following Eq. $4-5$ in each case. We can now plot the exclusion contours for a fixed $E_{\mathrm{br}}$ (recalling the relation between $\varepsilon_{\mathrm{br}}$ and $E_{\mathrm{br}}$ from Section 4.2.4) in a $\Phi_{\text {astro }}$ versus $\gamma_{\text {astro }}$ plot.

Figure 5.3 shows the $2 \sigma$ contours $\left(\Delta \chi^{2}=6.18\right)$ in black for several $E_{\mathrm{br}}$ values, with arrows pointing towards the allowed regions (of course, they point towards smaller $\Phi_{\text {astro }}$ ). In order to see the tension, we also plot the $1 \sigma$ (dashed) and $2 \sigma$ (dotted) allowed contours around the best fit value (stars) for each IceCube dataset, $\mu$-tracks in blue, Cascades in green ${ }^{3}$ and HESE in orange.

We can see that the tension with the cascade dataset evidently becomes $>2 \sigma$ for $E_{\mathrm{br}} \lesssim 20 \mathrm{TeV}$. For $E_{\mathrm{br}}=10 \mathrm{TeV}$, this tension grows to $\gtrsim 3 \sigma$, and for $E_{\mathrm{br}}=1 \mathrm{TeV}$ it even reaches $\sim 5 \sigma$. Of course, we cannot say for sure what its exact value is because we do not have the $3 \sigma$ (and higher) contours from IceCube, but we can certainly estimate that $E_{\mathrm{th}}=16 \mathrm{TeV}$ for Cascade events implies a tension at $\sim 3 \sigma \mathrm{CL}$. Not to mention, we are working with conservative assumptions for $E_{\text {th }}$ and neglecting any other $\gamma$-ray emission process at the sources (e.g. from leptonic processes like synchrotron radiation); in realistic scenarios this tension becomes even more significant statistically.

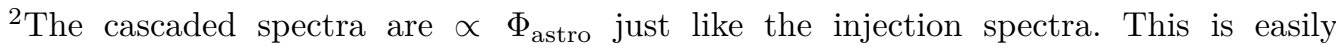
understood in terms of the individual cascade particles: a single photon injected at energy $\varepsilon_{\gamma}$ will reach the Earth at energy $E_{\gamma}$. The same is true for any number of photons injected at $\varepsilon_{\gamma}$, all reaching the Earth at $E_{\gamma}$, which is precisely what we do by multiplying the injection spectrum by a constant (i.e. changing the normalization).

${ }^{3}$ The 4 -year Cascade results show in Figure 5.3 are similar to the 6 -year dataset we have been using throughout this thesis. We only plot the 4-year data because we do not have the $2 \sigma$ contour in the $\Phi_{\text {astro- }} s_{h}$ plane for the 6-year data. 


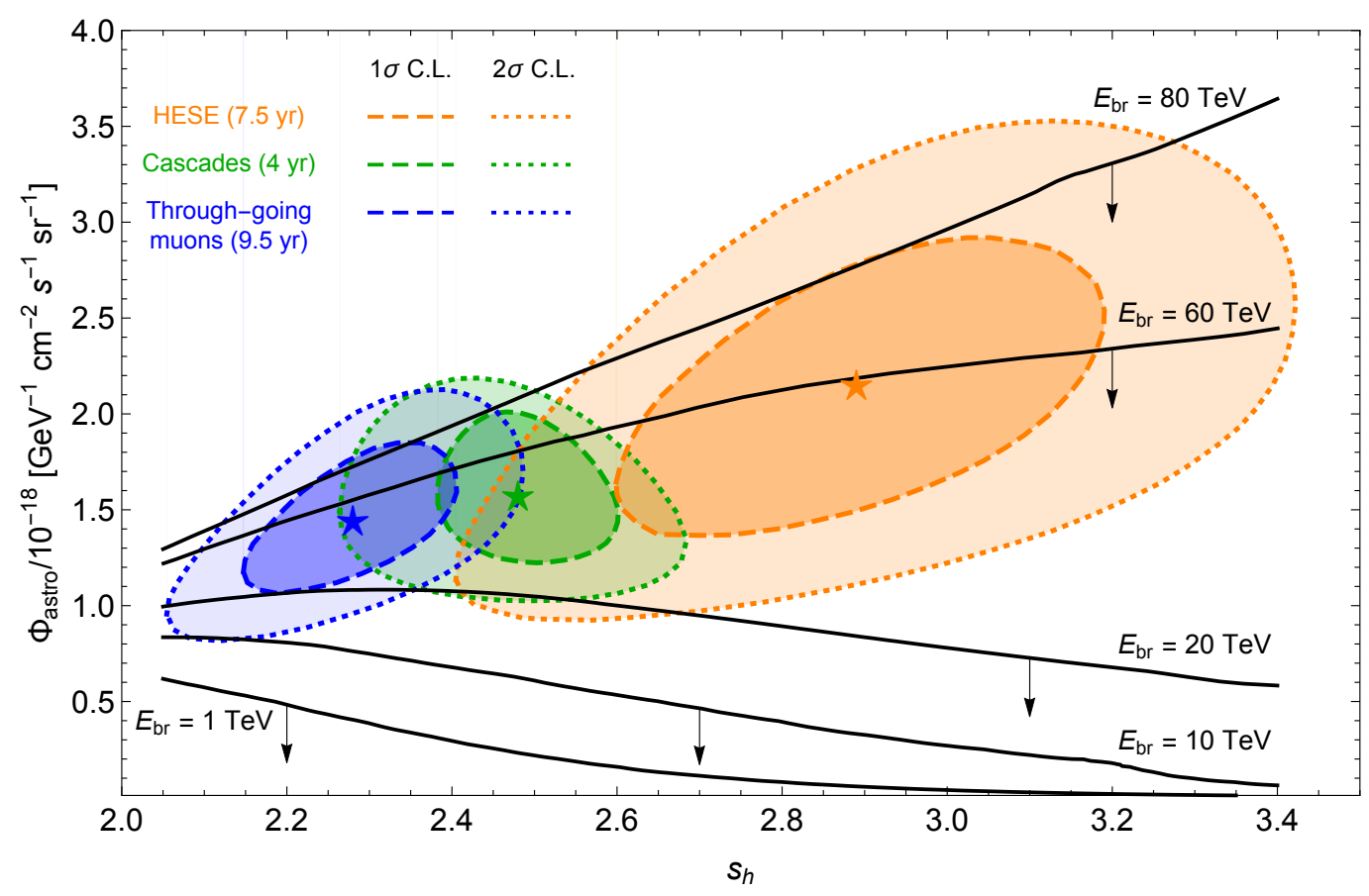

Figure 5.3: Limits for fixed $E_{\mathrm{br}}$ values in the $\Phi_{\text {astro }}-s_{h}$ plane are given by black contours, with arrows pointing towards allowed regions. The blue, green and orange contours are the $1 \sigma$ (dashed) and $2 \sigma$ (dotted) allowed regions for the 9.5y Tracks, 6y Cascade and 7.5y HESE IceCube datasets, respectively, with stars representing their best-fit values in Eq. 4-8. Since, $E_{\text {th }}=16 \mathrm{TeV}$ for the Cascade dataset, by assuming a change in spectral behaviour at $E_{\mathrm{br}}=10 \mathrm{TeV}$, we find a $\gtrsim 3 \sigma$ tension. This tension increases to $\sim 5 \sigma$ for $E_{\mathrm{br}}=1 \mathrm{TeV}$. Figure adapted from [79].

\section{2}

\section{Complementary Results and Discussions}

Finally, let's discuss our complementary analysis results from $\gamma$-ray flux integration above $50 \mathrm{GeV}$ as described in Section 4.2.2. We return to the $\varepsilon_{\mathrm{br}^{-}} s_{h}$ plane just as in Figure 5.1, this time establishing our $\gamma$-ray constraints without the use of a $\chi^{2}$ function, but instead of Eq. 4-7. Adopting the highest and lowest allowed normalizations at $1 \sigma$ CL for each dataset (same as in Figure 5.1), any $\left(\varepsilon_{\mathrm{br}}, s_{h}\right)$ pair satisfying $F_{\mathrm{cas}}^{>50 \mathrm{GeV}}\left(\varepsilon_{\mathrm{br}}, s_{h}\right) \geq(1-q) F_{\mathrm{EGB}}^{>50 \mathrm{GeV}}$ is directly excluded, with equality in this last expression determining the exclusion contours for a given value of $q$. The contours for $q=82 \%, 86 \%$ (best fit value) and $88 \%$ are all represented in Figure 5.4, with high norm results as solid colored lines and low norm results as dashed colored lines. We also represent the $E_{\mathrm{br}}=E_{\mathrm{th}}$ limits by gray contours with " $\nu$ "-labelled arrows. Invariably, all arrows point toward allowed regions. 


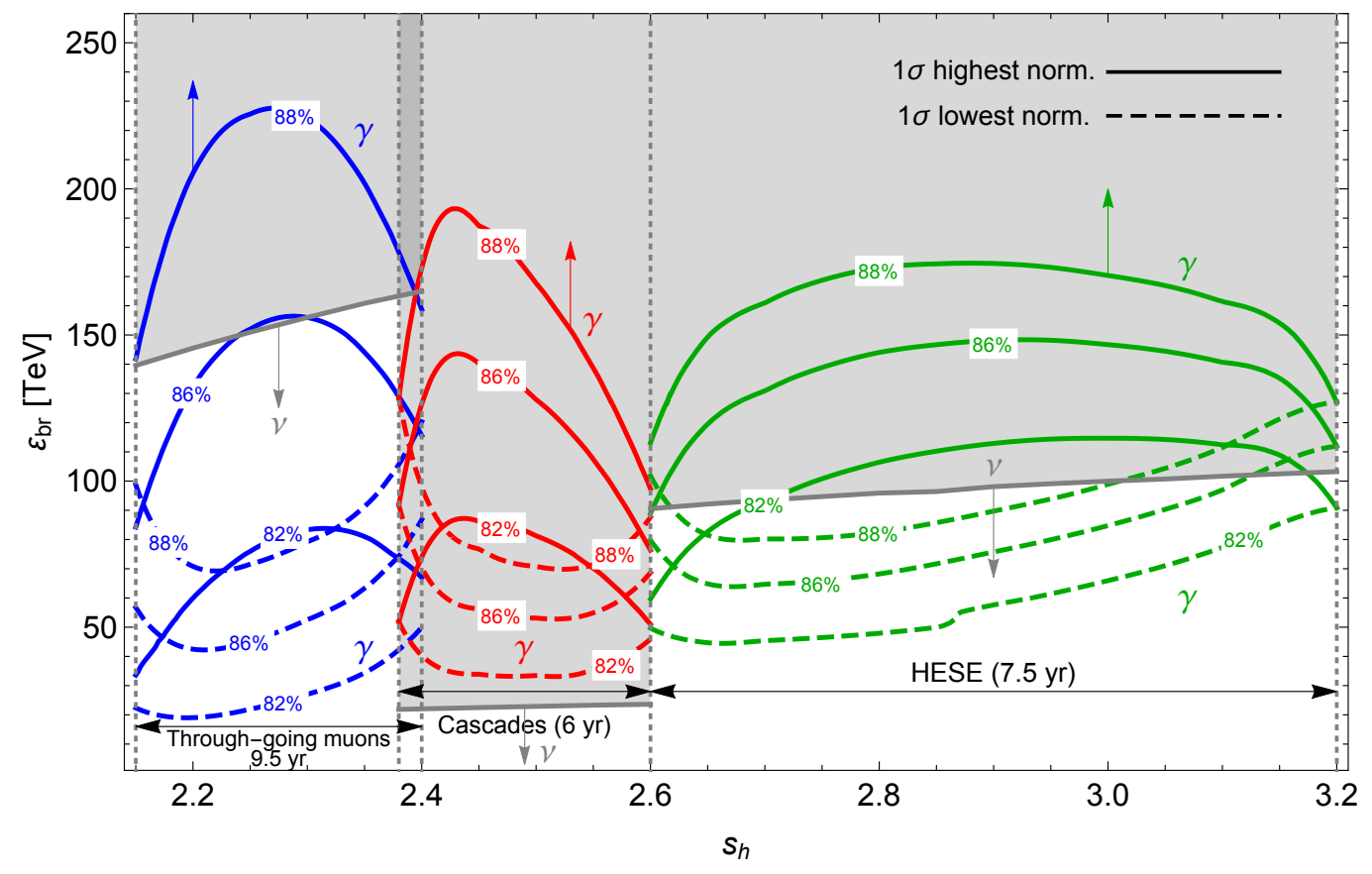

Figure 5.4: Exclusions from cascaded $\gamma$-ray flux integration (colored contours) as described in Section 4.2.2 for $q=82 \%, 86 \%$ and $88 \%$, with arrows pointing towards allowed regions. Contours for smallest(largest) normalization allowed at $1 \sigma$ CL are given by dashed(solid) colored lines. Gray contours indicate IceCube's thresholds for each dataset $\left(E_{\mathrm{br}}=E_{\mathrm{th}}\right)$. Similar to Figure 5.1, we see a multimessenger tension arising from the Cascade dataset. Figure reproduced from [79].

In agreement with our previous results from Figure 5.1, the through-going $\mu$-tracks (blue $\gamma$ contours) and HESE (green $\gamma$ contours) datasets enjoy a very similar parameter space region allowed by both neutrino and $\gamma$-ray constraints at $1 \sigma$ for the best fit value $q=86 \%$. The stricter $q=88 \%$ requirement is also marginally allowed by both at $1 \sigma$, although any increase in $q$ might result in tensions with HESE data. Still, the tension persists between the Cascade dataset and EGB constraints, even for $q=82 \%$. Please recall that this analysis is naturally less constraining than our main results because integration is not sensitive to the overall shape of the spectrum. Overshoots and undershoots of the EGB data points could compensate each other, even though such a scenario would be heavily excluded by our $\chi^{2}$ test.

With that said, we have successfully confirmed our previous results by means of this complementary analysis, which just serves to strengthen the evidence for a tension between IceCube and $\gamma$-ray data. Indeed, we are now left with the task of explaining this $\gtrsim 3 \sigma$ tension. This is the topic of this chapter's last section. 


\section{3 \\ Interpretations and Discussions}

Given all the arguments presented in the previous chapters and the current situation we find ourselves in, we can explore several ways to alleviate our newly quantified multimessenger tension:

( $i$ ) Damping the source's emission of $\gamma$-rays, but not neutrinos. In this case, we would probably be discovering a new type of source (or source environment) which is " $\gamma$-ray-opaque", meaning that only neutrinos can escape due to their small interaction probability, while high energy photons will almost always get absorbed before leaving. This eliminates (or damps significantly) the cascaded $\gamma$-ray contribution to the EGB, avoiding the tension. This solution, however, may present challenges in the model-building sense: on one hand the source must be dense enough to suppress $\gamma$-ray emission, on the other hand, the denser a source becomes, the harder it gets to accelerate charged particles (which are responsible for producing the neutrinos and $\gamma$-rays in the first place). Much work has been done in modelling such $\gamma$-ray-opaque source candidates, which include choked-jet GRBs [150, 151] and AGN cores $[152,153,154,155,156]$. MeV $\gamma$-ray and x-ray counterpart searches have also been suggested [100], since only lower energy photons would be capable of escaping their sources.

(ii) Source population distribution not resembling to the SFR cosmic evolution. Although the SFR is a natural assumption for $\mathcal{F}(z)$ in Eq. 2-13 (especially if a large fraction of them are GRBs or SBGs), some astrophysical objects follow very different $z$-distributions. BL Lacs are an example of a more locally concentrated population, with density peaking at $z=0$ shown in Figure B.2. $\gamma$-rays emitted closer to the Earth are less susceptible to being attenuated by the electromagnetic cascade, resulting in a displacement of the cascaded flux to higher energies. On the other hand, sources confined to the high- $z$ universe are guaranteed to fully develop the cascade, shifting the cascaded flux to lower energies. In either case, one can speculate that the resulting flux might avoid overshooting the EGB in the $10-100 \mathrm{GeV}$ range, which is the case for the SFR distribution. Alas, further investigations [80] have shown that this tension persists for any generic $\mathcal{F}(z)$.

(iii) Diffuse neutrino emissions coming from the Milky Way. In principle, it is possible that $\mathrm{CR}$ interactions with gas in the Galactic halo produces a quasi-isotropic diffuse neutrino flux which explains (at least partially) the 
IceCube excess $[157,158]$. However, this scenario predicts a comparable $\mathrm{PeV}$ contribution to the IGRB in tension with upper limits from the KASCADE [159] and HAWK [160] experiments. It is also disfavored since it requires a harder spectrum below $\varepsilon_{\mathrm{br}}\left(s_{l} \lesssim 2\right)$ than predicted by Galactic CR data $\left(s_{l} \sim 2.3-2.4\right)[42]$. As a side note, fluctuations around IceCube's isotropy may be accounted for by non-isotropic contributions from the Galactic Plane or from the so-called Fermi Bubbles [161].

(iv) Neutrino production from BSM processes. While heavy DM decay and annihilation (Galactic and extragalactic) have been extensively considered $[162,163]$ and updated $[164,165,166]$, other exotic scenarios such as neutrino decay $[167,168]$ could increase the $\gamma$-ray to neutrino ratio, increasing our tension and strengthening our results.

Finally, recall that we have been very conservative on our assumptions throughout this work. On top of assuming a minimal neutrino injection spectrum in Eq. 4-1, we neglect muon and meson cooling at the source, which can yield a larger ratio of produced $\gamma$-rays to neutrinos. Additional $\gamma$-ray emission unaccompanied by neutrinos can occur via leptonic processes such as synchrotron radiation, ICS, $\gamma \gamma$ annihilation and Bethe-Heitler pair production [169] $\left(p+\gamma \longrightarrow p+e^{+}+e^{-}\right)$, which is expected to dominate in AGN cores [170]. Furthermore, we have considered relatively conservative conventional EGB contributions; several higher estimates exist in the literature [132, 133, 134, 148]. In particular, we neglect the contribution from cosmogenic $\gamma$-ray production by UHECRs propagating through the intergalactic space, which could be significant at $\gtrsim 10 \mathrm{GeV}$ energies (see Figure 5 in [171]). Surely, the tension increases in more realistic scenarios, emphasising the need to further explore the aforementioned possibilities. With that said, we are now ready to express our closing remarks, recapping our original goals and evaluating how we have fulfilled them. 


\section{6}

\section{Final Remarks}

The discovery of astrophysical neutrinos by IceCube has opened up a new window to explore the most energetic and dense objects in the Universe. These cosmic messengers possess special properties that allow us to probe these environments better than ever, gaining information about their internal acceleration and neutrino production mechanisms. Searching for the sources of the neutrinos observed by IceCube has been a great challenge for many years, with point-source searches yielding null results so far.

With the help of a multimessenger approach, hints of a tension between neutrino and $\gamma$-ray data has led to speculations about the nature of these sources. In this context, we have established in this work the first quantitative results on this tension, under conservative assumptions regarding the ratio of produced gamma-rays to neutrinos. By comparing the expected bounds from IceCube and Fermi-LAT, we have found a $\gtrsim 3 \sigma$ tension between the "Cascades" IceCube dataset and the EGB, assuming that the single power-law fit for the astrophysical neutrino flux extends down to $10 \mathrm{TeV}$. This tension has been further confirmed in a complementary analysis in Section 5.2.

We have discussed a few possible interpretations for this result: $\gamma$-rayopaque sources, Galactic neutrino emission, BSM physics and different zdistribution of sources. The latter option seems to be unviable in view of recent results [80]. In fact, the refined analysis in [80] has shown that this tension is not only generic but also more robust. It persists when considering:

- All three EGB FG models (see Section 4.1),

- Separate nuisance parameters for blazars and radio galaxies in the $\chi^{2}$ definition,

- Gaussian and log-normal pull terms in the $\chi^{2}$,

- Updated conventional contributions from blazars [132], RGs [133] and SFGs [134],

- Only the $>10 \mathrm{GeV}$ part of the EGB, instead of the entire energy range.

In view of our current situation, some interesting prospects for the future of multimessenger astrophysics are suggested. Firstly, we emphasize the 
importance of extending the IceCube sensitivity to lower energies, lowering $E_{\mathrm{th}}$. The non-observation of a spectral break at lower energies would further increase this tension $(\sim 5 \sigma$ for a break at $1 \mathrm{TeV})$, eliminating any doubt that the tension exists. The much anticipated IceCube-Gen2 [19, 172, 173] upgrade will increase the instrumented volume of the current experiment by a factor of 10, deploying sparse DOMs which will increase the sensitivity mostly to higher energies ( $>$ EeV). A better low-energy resolution can be achieved by expanding IceCube's densely instrumented region called DeepCore. Still, the higher statistics of IceCube-Gen2 would allow for a better measurement of $\gamma_{\text {astro }}$ and $\Phi_{\text {astro }}$ which are crucial parameters in our analysis.

Other future experiments to look out for include KM3Net [174], a neutrino detector in the Mediterranean sea providing a complementary observation of the northern sky, HAWC [175], a water Cherenkov observatory capable of extending the EGB data beyond $>$ TeV, and LHAASO [176], pushing $\gamma$-ray observations even further to $\mathrm{PeV}$ energies (not to mention its superb sensitivity to CRs between $\mathrm{TeV}-\mathrm{EeV}$ ). These last two experiments will be particularly useful in constraining nearby sources, whose $\gamma$-ray cascades do not fully develop. We also encourage further assessments of the conventional EGB contributions, specially below $\sim \mathrm{GeV}$ energies where the RG and SFG contributions become significant, and so do their large uncertainties. Lastly, it is important that Fermi expands its high-energy EGB data into the multi-TeV range. Independent efforts have tried to achieve this [177]; however, it requires confirmation by the Fermi Collaboration.

All things considered, we are incredibly lucky that Nature has left us with so many exciting riddles in astrophysics, one of them being to explain this tension. The advancements of multimessenger searches predict a bright future for the field, with breakthroughs just waiting to be made as we push towards unexplored energies and higher sensitivities. I hope this dissertation has been helpful to you, whether you are experienced on the subject or an aspiring astrophysicist. Thank you for reading! 


\section{Bibliography}

[1] HESS, V. F. Über beobachtungen der durchdringenden strahlung bei sieben. Physik. Zeitschr., 13:1084-1091, 1912.

[2] ABDOllahI, S. ET. Al. [THE FERMi-LAT COlLABORATION]. A gamma-ray determination of the universe's star formation history. Science, 362 (6418):1031-1034, 2018.

[3] PENZIAS, A.; WILSON, R.. A measurement of excess antenna temperature at $4080 \mathrm{mc} / \mathrm{s}$. Astrophys. J., 142:419-421, 1965.

[4] GREISEN, K.. End to the cosmic-ray spectrum? Phys. Rev. Lett., 16:748-750, 1966.

[5] ZATSEPIN, G.; KUZMIN, V.. Upper limit of the spectrum of cosmic rays. Pis'ma Zh. Eksp. Teor. Fiz., 4:114-117, 1966.

[6] ZATSEPIN, G.; KUZMIN, V.. Upper limit of the spectrum of cosmic rays. JETP Lett., 4:78-80, 1966.

[7] BERESINSKY, V. S.; ZATSEPIN, G.. Cosmic rays at ultra high energies (neutrino?). Phys. Lett. B, 28:423-424, 1969.

[8] BERESINSKY, V. S.; ZATSEPIN, G.. Cosmic rays at ultra high energies (neutrino?). Sov. J. Nucl. Phys., 11:111, 1970.

[9] BEREZINSKY, V. S.; SMIRNOV, A. Y.. Cosmic neutrinos of ultrahigh energies and detection possibility. Astrophys. Space Sci., 32:461-482, 1975.

[10] FINKE, J. D. ET AL.. Constraints on the intergalactic magnetic field with gamma-ray observations of blazars. Astrophys. J., 814:20:1-14, 2015.

[11] AHARONIAN, F. A.; COPPI, P. S.; VOELK, H. J.. Very high energy gamma-rays from agn: Cascading on the cosmic background radiation fields and the formation of pair halos. Astrophys. J. Lett., 423:L5—L8, 1994. 
[12] ABBOT, B. P. ET AL. [LIGO SCIENTIFIC COLLABORATION AND VIRGO COLLABORATION]. Observation of gravitational waves from a binary black hole merger. Phys. Rev. Lett., 116:061102, 2016.

[13] AARTSEn M. G. ET AL. [ICECUBE COlLABORATION]. Astrophysical neutrinos and cosmic rays observed by icecube. Adv. Space Res., 62:2902-2930, 2018.

[14] AARTSEN, M. G. ET AL. [ICECUBE COLLABORATION]. First observation of pev-energy neutrinos with icecube. Phys. Rev. Lett., 111:021103, 2013.

[15] AARTSEN, M. G. ET AL. [ICECUBE COLLABORATION]. Evidence for high-energy extraterrestrial neutrinos at the icecube detector. Science, 342 (6161):1242856, 2013.

[16] AARTSEn, M. G. eT Al. [ICECUBe COLLABORATION]. Search for a diffuse flux of astrophysical muon neutrinos with the icecube 59-string configuration. Phys. Rev. D, 89:062007, 2014.

[17] HAACK, C.; WIEBUSCH, C.. A measurement of the diffuse astrophysical muon neutrino flux using eight years of icecube data. In: 35TH INTERNATIONAL COSMIC RAY CONFERENCE-ICRC2017, p. 30-37, Bexco, Busan, Korea, 10-20 July, 2017.

[18] AARTSEN, M. G. ET AL. [ICECUBE COLLABORATION]. Observation of high-energy astrophysical neutrinos in three years of icecube data. Phys. Rev. Lett., 113:101101, 2014.

[19] VAN SANTEN, J.. Highlights from icecube and prospects for icecube-gen2. In: 35TH INTERNATIONAL COSMIC RAY CONFERENCE-ICRC2017, volumen 301, p. 1110, Bexco, Busan, Korea, 10-20 July, 2017.

[20] BUSTAMANTE, M.; AHLERS, M.. Inferring the flavor of highenergy astrophysical neutrinos at their sources. Phys. Rev. Lett., 122:241101, 2019.

[21] AARTSEN, M. G. ET AL. [ICECUBE COLLABORATION]. A combined maximum-likelihood analysis of the high-energy astrophysical neutrino flux measured with icecube. Astrophysical Journal, 809:98, 2015. 
[22] BUSTAMANTE M.; CONNOLlY, A.. Extracting the energydependent neutrino-nucleon cross section above 10 tev using icecube showers. Phys. Rev. Lett., 122:041101, 2019.

[23] AartSen, M. G. eT Al. [ICECUBE COllabORATIOn]. Search for sources of astrophysical neutrinos using seven years of icecube cascade events. Astrophys. J., 886:12, 2019.

[24] AARTSEN, M. G. ET AL. [ICECUBE COLLABORATION]. Energy reconstruction methods in the icecube neutrino telescope. JINST, 9:P03009, 2014.

[25] GLASHOW, S. L.; ZHANG, B.. Resonant scattering of antineutrinos. Phys. Rev., 118:316-317, 1960.

[26] BHATTACHARYA, A.; GANDHI, R.; RODEJOHANN, W.; WATANABE, A.. The glashow resonance at icecube: signatures, event rates and $p p$ vs. $p \gamma$ interactions. JCAP, 10:017, 2011.

[27] HAACK, C.; LU, L.; YUAN, T. (ON BEHALF OF THE ICECUBE COLLABORATION). Detection of a neutrino event at the glashow resonance energy in icecube. In: EUROPEAN PHYSICAL SOCIETY CONFERENCE ON HIGH ENERGY PHYSICS-EPS-HEP2019, Ghent, Belgium, July 10th-17th, 2019.

[28] AARTSEN, M. G. ET AL. [ICECUBE COLLABORATION]. Searches for extended and point-like neutrino sources with four years of icecube data. Astrophys. J., 796:109:14, 2014.

[29] ICECUBE COLLABORATION. "IceCube Media Gallery". IceCube, 2020. Access in: July 2020.

[30] STACHURSKA, J. (ON BEHALF OF THE ICECUBE COLLABORATION). First double cascade tau neutrino candidates in icecube and a new measurement of the flavor composition. In: 36TH INTERNATIONAL COSMIC RAY CONFERENCE-ICRC2019, p. 1-7, Madison, WI, U.S.A., July 24th-August 1st, 2019.

[31] COWEN, D. F. (ON BEHALF OF THE ICECUBE COLLABORATION). Tau neutrinos in icecube. J. Phys. Conf. Ser., 60:227-230, 2007.

[32] URRY, C. M.. Agn unification: An update. In: AGN PHYSICS WITH THE SLOAN DIGITAL SKY SURVEY, p. 49-60, Princeton, NJ, U.S.A., July, 2004. 
[33] RIEGER, F. M.. Uhe cosmic rays and agn jets. In: $7 \mathrm{TH} \mathrm{HIGH}$ ENERGY PHENOMENA IN RELATIVISTIC OUTFLOWS (HEPRO VII), p. 1-12, Barcelona, Spain, 9-12 July, 2019.

[34] ESSEY, W.; KALASHEV, O. E.; KUSENKO, A.; BEACOM, J. F.. Secondary photons and neutrinos from cosmic rays produced by distant blazars. Phys. Rev. Lett., 104:141102, 2010.

[35] KALASHEV, O. E.; KUSENKO, A.; ESSEY, W.. Pev neutrinos from intergalactic interactions of cosmic rays emitted by active galactic nuclei. Phys. Rev. Lett., 111:041103, 2013.

[36] MURASE, K.; INOUE, Y.; DERMER, C. D.. Diffuse neutrino intensity from the inner jets of active galactic nuclei: Impacts of external photon fields and the blazar sequence. Phys. Rev. D, 90:023007, 2014.

[37] HOOPER, D.. A case for radio galaxies as the sources of icecube's astrophysical neutrino flux. JCAP, 09(002):1-15, 2016.

[38] BOETTCHER, M.. Modeling the spectral energy distributions and variability of blazars. In: 2012 FERMI \& JANSKY PROCEEDINGS ECONF C111110, p. 1-9, Harbourtowne Conference Center, St Michaels, MD, USA 10-12 November, 2012.

[39] MIHOS, J. C.; HERNQUIST, L.. Gasdynamics and starbursts in major mergers. Astrophys. J., 464:641-663, 1996.

[40] HE, H. -N. ET AL.. Diffuse pev neutrino emission from ultraluminous infrared galaxies. Phys. Rev. D, 87:063011, 2013.

[41] LOEB, A.; WAXMAN, E.. The cumulative bakground of highenergy neutrinos from starburst galaxies. JCAP, 05(003):1-8, 2006.

[42] MURASE, K.; AHLERS, M.; LACKI, B. C.. Testing the hadronuclear origin of pev neutrinos observed with icecube. Phys. Rev. D, 88:121301, 2013.

[43] ROMERO, G. E.; TORRES, D. F.. Signatures of hadronic cosmic rays in starbursts? high-energy photons and neutrinos from ngc 253. Astrophys. J., 586:L33-L36, 2003.

[44] LACKI, B. C. ET AL.. On the gev and tev detections of the starburst galaxies $\mathbf{m 8 2}$ and ngc 253. Astrophys. J., 734:107:1-14, 2011. 
[45] MÉSZÁROS, P.. Neutrino Astronomy - Current Status, Future Prospects, chapter 1. Gamma Ray Bursts as Neutrino Sources. World Scientific, 2017.

[46] WAXMAN, E.; BAHCALL, J.. High energy neutrinos from cosmological gamma-ray burst fireballs. Phys. Rev. Lett., 78:2292-2295, 1997.

[47] WAXMAN, E.; BAHCALL, J.. Neutrino afterglow from gamma-ray bursts: $\sim 10^{18} \mathrm{ev}$. Astrophys. J., 541:707-711, 2000.

[48] GABICI, S.; GAGGERO, D.; ZANDANEL, F.. Can supernova remnants accelerate protons up to pev energies? In: 28TH RENCONTRES DE BLOIS ON PARTICLE PHYSICS AND COSMOLOGY, p. 1-8, Blois, France, May 29th-June 3rd, 2016.

[49] ALVAREZ-MUÑIZ, J.; HALZEN, F.. Possible high-energy neutrinos from the cosmic accelerator $\mathrm{rx} \mathbf{j} 1713.7-3946$. Astrophys. J., 576:L33-L36, 2002.

[50] CAVASINNI, V.; GRASSO, D.; MACCIONE, L.. Tev neutrinos from supernova remnants embedded in giant molecular clouds. Astropart. Phys., 26:41-49, 2006.

[51] GONZALEZ-GARCIA, M. C.; HALZEN, F.; NIRO, V.. Reevaluation of the prospect of observing neutrinos from galactic sources in the light of recent results in gamma ray and neutrino astronomy. Astropart. Phys., 57-58:39-48, 2014.

[52] VISSANI, F.; AHARONIAN, F.; SAHAKYAN, N.. On the detectability of high-energy galactic neutrino sources. Astropart. Phys., 34:778783, 2011.

[53] FARRAR, G. R.; GRUZINOV, A.. Giant agn flares and cosmic ray bursts. Astrophys. J., 693:329-332, 2009.

[54] LUNARDINI, C.; WINTER, W.. High energy neutrinos from the tidal disruption of stars. Phys. Rev. D, 95:123001, 2017.

[55] AARTSEn, M. G. et Al. [ICECUBe COlLabORATION]. Search for astrophysical sources of neutrinos using cascade events in icecube. Astrophys. J., 846:136:1-12, 2017.

[56] ABDOLLAHI, S. ET AL. [THE FERMI-LAT COLLABORATION]. Fermi large area telescope fourth source catalog. ApJS, 247:33:1-37, 2020. 
[57] WAKELY, S. P.; HORAN, D.. "TeVCat: online catalogue of TeV sources". TeVCat, 2018. Access in: August 2020.

[58] VORUGANTI, A. ET AL.. "Gamma-Cat: online catalogue of Gamma-ray sources". Gamma-Cat, 2018. Access in: August 2020.

[59] AARTSEN, M. G. ET AL. [ICECUBE COllabORATION]. Timeintegrated neutrino source searches with 10 years of icecube data. Phys. Rev. Lett., 124:051103, 2020.

[60] AARTSEN, M. G. ET AL. [ICECUBE COLLABORATION]. All-sky search for time-integrated neutrino emission from astrophysical sources with 7 yr of icecube data. Astrophys. J., 835:151:1-15, 2017.

[61] AARTSEN, M. G. ET AL. [ICECUBE COLLABORATION]. Searches for extended and point-like neutrino sources with four years of icecube data. Astrophys. J., 796:109:1-14, 2014.

[62] SCHATTO, K.. Stacked searches for high-energy neutrinos from blazars with IceCube. Dissertation, Johannes Gutenberg-Universität Mainz, 55128 Mainz, Germany, 2014.

[63] AARTSEN, M. G. ET AL. [ICECUBE COLLABORATION]. Search for time-independent neutrino emission from astrophysical sources with 3 yr of icecube data. Astrophys. J., 779:132:1-17, 2013.

[64] ABBASI, R. ET AL. [ICECUBE COLlabORATION]. Time-integrated searches for point-like sources of neutrinos with the 40-string icecube detector. Astrophys. J., 732:18:1-16, 2011.

[65] ACHTERBERG, A. ET AL. [ICECUBE COlLABORATION]. On the selection of agn neutrino source candidates for a source stacking analysis with neutrino telescopes. Astropart. Phys., 26:282-300, 2006.

[66] SU, M.; SLATYER, T. R.; FINKBEINER, D. P.. Giant gamma-ray bubbles from fermi-lat: Agn activity or bipolar galactic wind? Astrophys. J., 724:1044-1082, 2010.

[67] LUNARDINI, C. ET AL.. Neutrino events at icecube and the fermi bubbles. Phys. Rev. D, 90:023016, 2014. 
[68] THE ICECUBE COLLABORATION ET AL.. Multimessenger observations of a flaring blazar coincident with high-energy neutrino icecube-170922a. Science, 361:1-8, 2018.

[69] AARTSEN, M. G. ET AL. [ICECUBE COlLABORATION]. Neutrino emission from the direction of the blazar txs $0506+056$ prior to the icecube-170922a alert. Science, 361:147-151, 2018.

[70] HOOPER, D.; LINDEN, T.; VIEREGG, A.. Active galactic nuclei and the origin of icecube's diffuse neutrino flux. JCAP, 02:012:1-20, 2019.

[71] BRAUN, J. ET AL.. Time-dependent point source search methods in high energy neutrino astronomy. Astropart. Phys., 33:175-181, 2010.

[72] BRAUN, J. ET AL.. Methods for point source analysis in high energy neutrino telescopes. Astropart. Phys., 29:299-305, 2008.

[73] AARTSEN, M. G. ET AL. [ICECUBE COLLABORATION]. Extending the search for muon neutrinos coincident with gamma-ray bursts in icecube data. Astrophys. J., 843:112:1-13, 2017.

[74] AARTSEN, M. G. ET AL. [ICECUBE COLLABORATION]. An all-sky search for three flavors of neutrinos from gamma-ray bursts with the icecube neutrino observatory. Astrophys. J., 824:115:113, 2016.

[75] AARTSEN, M. G. ET AL. [ICECUBE COLLABORATION]. Search for prompt neutrino emission from gamma-ray bursts with icecube. Astrophys. J. Lett., 805:L5:1-7, 2015.

[76] ABBASI, R. U. ET AL. [ICECUBE COLLABORATION]. Search for muon neutrinos from gamma-ray bursts with the icecube neutrino telescope. Astrophys. J., 710:346-359, 2010.

[77] THE ICECUBE, PIERRE AUGER AND TELESCOPE ARRAY COLLABORATIONS. Search for correlations between the arrival directions of icecube neutrino events and ultrahigh-energy cosmic rays detected by the pierre auger observatory and the telescope array. JCAP, 037:1-33, 2016.

[78] WAXMAN, E.; BAHCALL, J.. High energy neutrinos from astrophysical sources: An upper bound. Phys. Rev. D, 59:023002, 1998. 
[79] CAPANEMA, A.; ESMAILI, A.; MURASE, K.. New constraints on the origin of medium-energy neutrinos observed by icecube. Phys. Rev. D, 101:103012, 2020.

[80] CAPANEMA, A.; ESMAILI, A.; SERPICO, P.. Where do icecube neutrinos come from? hints from the diffuse gamma-ray flux. arXiv:2007.07911 [hep-ph], 2020.

[81] ABBASI, R. ET AL. [ICECUBE COLLABORATION]. Extending the search for neutrino point sources with icecube above the horizon. Phys. Rev. Lett., 103:221102, 2009.

[82] AARTSEN, M. G. ET AL. [ICECUBE COLLABORATION]. Development of a general analysis and unfolding scheme and its application to measure the energy spectrum of atmospheric neutrinos with icecube. Eur. Phys. J. C, 75 (116), 2015.

[83] LIPARI, P.. Lepton spectra in the earth's atmosphere. Astroparticle Physics, 1:195-227, 1993.

[84] SCHÖNERT, S. ET AL.. Vetoing atmospheric neutrinos in a high energy neutrino telescope. Phys. Rev. D, 79:043009, 2009.

[85] GAISSER, T. K. ET AL.. Generalized self-veto probability for atmospheric neutrinos. Phys. Rev. D, 90:023009, 2014.

[86] AARTSEN, M. G. ET AL.. Atmospheric and astrophysical neutrinos above 1 tev interacting in icecube. Phys. Rev. D, 91:022001, 2015.

[87] KOOPER, C.; GIANG, W; KURAHASHI, N.. Observation of astrophysical neutrinos in four years of icecube data. In: THE 34TH INTERNATIONAL COSMIC RAY CONFERENCE: CONTRIBUTIONS TO ICRC 2015 PART II: ATMOSPHERIC AND ASTROPHYSICAL DIFFUSE NEUTRINO SEARCHES OF ALL FLAVORS, p. 45-52, The Hague, The Netherlands, 30 July-6 August, 2015.

[88] KOOPER, C.. Observation of astrophysical neutrinos in six years of icecube data. In: 35TH INTERNATIONAL COSMIC RAY CONFERENCE-ICRC2017(981), p. 1-8, Bexco, Busan, Korea, 10-20 July, 2017.

[89] SCHNEIDER, A.. Characterization of the astrophysical diffuse neutrino flux with icecube high-energy starting events. In: 
36TH INTERNATIONAL COSMIC RAY CONFERENCE-ICRC2019, p. 1-8, Madison, WI, U.S.A., July 24th-August 1st, 2019.

[90] NIEDERHAUSEN, H.; LESIAK-BZDAK, M.; STOESS, A.. High energy astrophysical neutrino flux characteristics for neutrinoinduced cascades using ic79 and ic86-string icecube configurations events. In: THE 34TH INTERNATIONAL COSMIC RAY CONFERENCE-ICRC2015, p. 1-8, The Hague, The Netherlands, 30 July-6 August, 2015.

[91] NIEDERHAUSEN, H.; LESIAK-BZDAK, M.; STOESS, A.. High energy astrophysical neutrino flux measurement using neutrinoinduced cascades observed in 4 years of icecube data. In: $35 \mathrm{TH}$ INTERNATIONAL COSMIC RAY CONFERENCE-ICRC2017(981), p. 1-8, Bexco, Busan, Korea, 10—20 July, 2017.

[92] AARTSEN, M. G. ET AL. (ICECUBE COLLABORATION). Characteristics of the diffuse astrophysical electron and tau neutrino flux with six years of icecube high energy cascade data. Phys. Rev. Lett., 125:121104, 2020.

[93] AARTSEN, M. G. ET AL. [ICECUBE COLLABORATION]. Evidence for astrophysical muon neutrinos from the northern sky with icecube. Phys. Rev. Lett., 115:081102, 2015.

[94] AARTSEN, M. G. ET AL. [ICECUBE COLLABORATION]. Observation and characterization of a cosmic muon neutrino flux from the northern hemisphere using six years of icecube data. Astrphys. J., 833, 2016.

[95] STETTNER, J.. Measurement of the diffuse astrophysical muonneutrino spectrum with ten years of icecube data. In: $36 \mathrm{TH}$ INTERNATIONAL COSMIC RAY CONFERENCE-ICRC2019, p. 1-7, Madison, WI, U.S.A., July 24th-August 1st, 2019.

[96] MURASE, K.. On the origin of high-energy cosmic neutrinos. AIP Conference Proceedings, 1666:040006, 2015.

[97] GAISSER, T.K.; ENGEL, R.; RESCONI, E.. Cosmic Rays and Particle Physics. Cambridge University Press, Cambridge, UK, 2nd edition, 2006.

[98] LOEB, A.; WAXMAN, E.. The cumulative background of highenergy neutrinos from starburst galaxies. JCAP, 05:003, 2006. 
[99] MURASE, K.; INOUE, S.; NAGATAKI, S.. Cosmic rays above the second knee from clusters of galaxies and associated highenergy neutrino emission. Astrophys. J., 689:L105, 2008.

[100] MURASE, K.; GUETTA, D.; AHLERS, M.. Hidden cosmic-ray accelerators as an origin of tev-pev cosmic neutrinos. Phys. Rev. Lett., 116:071101, 2016.

[101] GIUNTI, C.; KIM, C.; LEE, U.. When do neutrinos really oscillate? quantum mechanics of neutrino oscillations. Phys. Rev. D, 44 (11):3635-3640, 1991.

[102] ESTEBAN, I. ET AL.. The fate of hints: updated global analysis of three-flavor neutrino oscillations. JHEP, 09:178, 2020.

[103] ESTEBAN, I. ET AL.. "NuFIT 5.0 (2020)". NuFIT, 2020. Access in: October 2020.

[104] GIUNTI, C.; KIM, C.. Coherence of neutrino oscillations in the wave packet approach. Phys. Rev. D, 58:017301, 1998.

[105] ATHAR, H.; JEŻABEK, M.; YASUDA, O.. Effects of neutrino mixing on high-energy cosmic neutrino flux. Phys. Rev. D, 62:103007, 2000.

[106] BEREZINSKY, V.; KALASHEV, O.. High-energy electromagnetic cascades in extragalactic space: Physics and features. Phys. Rev. D, 94:023007, 2016.

[107] CAIRNS, J.. Developing the theory of flux limits from $\gamma$-ray cascades. arXiv:0705.2760 [astro-ph], 2007.

[108] BEREZINSKY, V. S.; BULANOV, S. V.; DOGIEL, V. A.; GINZBURG, V. L.(ED.); PTUSKIN, V. S.. Astrophysics of cosmic rays. North-Holland, Amsterdam, Netherlands, 1st edition, 1990.

[109] BLANCO, C.. $\gamma$-cascade: A simple program to compute cosmological gamma-ray propagation. JCAP, 01:13, 2019.

[110] MATHER, J. ET AL.. Measurement of the cosmic microwave background spectrum by the cobe firas instrument. Astrophys. J., 420:439-444, 1994.

[111] FIXSEN, D. ET AL.. The cosmic microwave background spectrum from the full cobe firas data set. Astrophys. J., 473:576-587, 1996. 
[112] FIXSEN, D.. The temperature of the cosmic microwave background. Astrophys. J., 707:916-920, 2019.

[113] PLANCK COLLABORATION. "Planck 2018 Results". Planck Publications, 2018. Access in: April 2020.

[114] COORAY, A.. Extragalactic background light: Measurements and applications. Roy. Soc. Op. Sci., 3:150555, 2016.

[115] DOMÍNGUEZ, A. ET AL.. Extragalactic background light inferred from aegis galaxy-sed-type fractions. Mon. Not. R. Astron. Soc., 410:2556-2578, 2011.

[116] FINKE, J. D.; RAZZAQUE, S.; DERMER, C. D.. Modeling the extragalactic background light from stars and dust. Astrophys. J., 712:238-249, 2010.

[117] FRANCESCHINI, A.; RODIGHIERO, G.; VACCARI, M.. The extragalactic optical-infrared background radiations, their time evolution and the cosmic photon-photon opacity. Astron. Astrophys., 487:837852, 2008.

[118] GILMORE, R. C.; SOMERVILLE, R. S.; PRIMACK, J. R.; DOMÍNGUEZ, A.. Semi-analytic modeling of the ebl and consequences for extragalactic gamma-ray spectra. Mon. Not. R. Astron. Soc., 422:31893207, 2012.

[119] KNEISKE, T. M.; DOLE, H.. A lower-limit flux for the extragalactic background light. Astron. Astrophys., 515:A19, 2010.

[120] SIGL, G.. Astroparticle Physics: Theory and Phenomenology. Atlantis Press, Hamburg, Germany, 1st edition, 2017.

[121] MOTS, J. W.; OLSEN, H. A.; KOCH, H. W.. Pair production by photons. Rev. Mod. Phys., 41:581-639, 1969.

[122] BLUMENTHAL, G. R.; GOULD, R. J.. Bremsstrahlung, synchrotron radiation, and compton scattering of high-energy electrons traversing dilute gases. Rev. Mod. Phys., 42:237-270, 1970.

[123] BREIT, G.; WHEELER, J. A.. Collision of two light quanta. Phys. Rev., 46:1087-1091, 1934. 
[124] PESKIN, M. E.; SCHROEDER, D. V.. An Introduction to Quantum Field Theory. Addison-Wesley Publishing Company, Boston, (MA, USA), 1st edition, 1995.

[125] LONGAIR, M. S.. High Energy Astrophysics. Cambridge University Press, Cambridge, (NY, USA), 3rd edition, 2011.

[126] ACKERMANN, M. ET AL. [THE FERMI LAT COLLABORATION]. The spectrum of isotropic diffuse gamma-ray emission between 100 mev and 820 gev. Astrophys. J., 799:86, 2015.

[127] ACKERMANN, M. ET AL. [THE FERMI LAT COLLABORATION]. Resolving the extragalactic $\gamma$-ray background above 50 gev with fermi-lat. Phys. Rev. Lett., 116:151105, 2016.

[128] ACKERMANN, M. ET AL.. 2fhl: The second catalog of hard fermilat sources. Astrophys. J., 222:1, 2006.

[129] AJELLO, M. ET AL.. The origin of the extragalactic gammaray background and implications for dark matter annihilation. Astrophys. J., 800:L27, 2015.

[130] INOUE, Y.. Contribution of gamma-ray-loud radio galaxies' core emissions to the cosmic mev and gev gamma-ray background radiation. Astrophys. J., 733:66, 2011.

[131] ACKERMANN, M. ET AL. [THE FERMI LAT COLLABORATION]. Gev observations of star-forming galaxies with Fermi lat. Astrophys. J., 755:164, 2012.

[132] QU, Y.; ZENG, H.; YAN, D.. Gamma-ray luminosity function of bl lac objects and contribution to the extragalactic gamma-ray background. Mon. Not. Roy. Astron. Soc., 490:758-765, 2019.

[133] DI MAURO, M. ET AL.. Diffuse $\gamma$-ray emission from misaligned active galactic nuclei. Astrophys. J., 780:161, 2014.

[134] AJELLO, M. ET AL.. The $\gamma$-ray emission of star-forming galaxies. Astrophys. J., 894:88, 2020.

[135] CUOCO, A.; KOMATSU, E.; SIEGAL-GASKINS, J. M.. Joint anisotropy and source count constraints on the contribution of blazars to the diffuse gamma-ray background. Phys. Rev. D, 86:063004, 2012. 
[136] HARDINGA, J. P.; ABAZAJIAN, K. N.. Models of the contribution of blazars to the anisotropy of the extragalactic diffuse gamma-ray background. JCAP, 11:026, 2012.

[137] ABDO, A. A. ET AL. [THE FERMI-LAT COLLABORATION]. The fermilat high-latitude survey: Source count distributions and the origin of the extragalactic diffuse background. Astrophys. J., 720:435-453, 2010.

[138] AJELLO, M. ET AL.. The luminosity function of fermi-detected flat-spectrum radio quasars. Astrophys. J., 751:108, 2012.

[139] KESHET, U. ET AL.. Gamma-rays from intergalactic shocks. Astrophys. J., 585:128-150, 2003.

[140] GABICI, S.; BLASI, P.. The gamma ray background from large scale structure formation. Astropart. Phys., 19:679-689, 2003.

[141] BLANCO, C.; HOOPER, D.. Constraints on decaying dark matter from the isotropic gamma-ray background. JCAP, 03:019, 2019.

[142] FELDMANN, R.; HOOPER, D.; GNEDIN, N. Y.. Circum-galactic gas and the isotropic gamma ray background. Astrophys. J., 763:21, 2013.

[143] KESHET, U.; WAXMAN, E.; LOEB, A.. The case for a low extragalactic gamma-ray background. JCAP, 04:006, 2004.

[144] MOSKALENKO, I. V.; PORTER, T. A.. Isotropic gamma-ray background: Cosmic-ray induced albedo from debris in the solar system? Astrophys. J., 692:54-57, 2009.

[145] MOSKALENKO, I. V. ET AL.. A celestial gamma-ray foreground due to the albedo of small solar system bodies and a remote probe of the interstellar cosmic-ray spectrum. Astrophys. J., 681:1708-1716, 2008.

[146] ORLANDO, E.; STRONG, A. W.. Gamma rays from halos around stars and the sun. Astrophys. Space Sci., 309:359-363, 2007.

[147] ORLANDO, E.; STRONG, A. W.. Gamma-ray emission from the solar halo and disk: a study with egret data. Astron. Astrophys., 480:847-857, 2008. 
[148] CALORE, F.; DI MAURO, M; DONATO, F.. Diffuse $\gamma$-ray emission from galactic pulsars. Astrophys. J., 796:14, 2014.

[149] ZYLA, P. A. ET AL. (PARTICLE DATA GROUP). Review of particle physics. Prog. Theor. Exp. Phys., 2020(8):083C01, 2020.

[150] TAMBORRA, I.; ANDO, S.. Inspecting the supernova-gammaray-burst connection with high-energy neutrinos. Phys. Rev. D, 93:053010, 2016.

[151] SENNO, N.; MURASE, K.; MESZAROS, P.. Choked jets and lowluminosity gamma-ray bursts as hidden neutrino sources. Phys. Rev. D, 93:083003, 2016.

[152] STECKER, F. W.. Ice cube observed pev neutrinos from agn cores. Phys. Rev. D, 88:047301, 2013.

[153] KALASHEV, O.; SEMIKOZ D.; TKACHEV, I.. Neutrinos in icecube from active galactic nuclei. JETP, 120:541-548, 2015.

[154] INOUE, Y., KHANGULYAN, D.; INOUE, S.; DOI, A.. On high-energy particles in accretion disk coronae of supermassive black holes: Implications for mev gamma-rays and high-energy neutrinos from agn cores. Astrophys. J., 880:40, 2019.

[155] MURASE, K.; KIMURA, S. S.; MESZAROS, P.. Hidden cores of active galactic nuclei as the origin of medium-energy neutrinos: Critical tests with the mev gamma-ray connection. Phys. Rev. Lett., 125:011101, 2020.

[156] KIMURA, S. S.; MURASE, K.; MÉSZÁROS, P.. Multi-messenger tests of cosmic-ray acceleration in radiatively inefficient accretion flows. Phys. Rev. D, 100:083014, 2019.

[157] TAYLOR, A. M.; GABICI, S.; AHARONIAN, F.. A galactic halo origin of the neutrinos detected by icecube. Phys. Rev. D, 89:103003, 2014.

[158] LIU, R. ET AL.. Gamma-ray production in the extended halo of the galaxy and possible implications for the origin of galactic cosmic rays. Astrophys. J., 871:40, 2019.

[159] SCHATZ, G. ET AL. [KASCADE COLLABORATION]. Search for extremely high energy gamma rays with the kascade experiment. In: 28TH INTERNATIONAL COSMIC RAY CONFERENCE - ICRC2003, p. 2293-2296, Tsukuba, Japan, 31 July-7 August, 2003. 
[160] HARDING, J. P. (FOR THE HAWC COLLABORATION). Constraints on the diffuse gamma-ray background with hawc. In: 36TH INTERNATIONAL COSMIC RAY CONFERENCE - ICRC2019(691), p. 1-7, Madison, WI, U.S.A., July 24th-August 1st, 2019.

[161] AHLERS, M.; MURASE, K.. Probing the galactic origin of the icecube excess with gamma-rays. Phys. Rev. D, 90:023010, 2014.

[162] ESMAILI, A.; SERPICO, P. D.. Are icecube neutrinos unveiling pevscale decaying dark matter? JCAP, 11:054, 2013.

[163] FELDSTEIN, B.; KUSENKO, A.; MATSUMOTO, S.; YANAGIDA, T. T.. Neutrinos at icecube from heavy decaying dark matter. Phys. Rev. D, 88:015004, 2013.

[164] ESMAILI, A.; KANG, S. K.; SERPICO, P. D.. Icecube events and decaying dark matter: hints and constraints. JCAP, 12:054, 2014.

[165] BHATTACHARYA, A.; ESMAILI, A.; PALOMARES-RUIZ, S.; SARCEVIC, I.. Update on decaying and annihilating heavy dark matter with the 6-year icecube hese data. JCAP, 05:051, 2019.

[166] CHIANESE, M.; FIORILLO, D. F. G.; MIELE, G.; MORISI, S.; PISANTI, O.. Decaying dark matter at icecube and its signature on high energy gamma experiments. JCAP, 11:046, 2019.

[167] DENTON, P. B.; TAMBORRA, I.. Invisible neutrino decay resolves icecube's track and cascade tension. Phys. Rev. Lett., 121:121802, 2018.

[168] BUSTAMANTE, M.; BEACOM, J. F.; MURASE, K.. Testing decay of astrophysical neutrinos with incomplete information. Phys. Rev. D, 95:063013, 2017.

[169] BETHE, H.; HEITLER, W.. On the stopping of fast particles and on the creation of positive electrons. Proc. Roy. Soc. Lond. A, 146:856:83-112, 1934.

[170] MURASE, K.; KIMURA, S. S.; MÉSZÉROS, P.. Hidden cores of active galactic nuclei as the origin of medium-energy neutrinos: Critical tests with the mev gamma-ray connection. Phys. Rev. Lett., 125:011101, 2020. 
[171] CHOLIS, I.; HOOPER, D.; MCDERMOTT, S. D.. Dissecting the gamma-ray background in search of dark matter. JCAP, 02(014):1-24, 2014.

[172] AARTSEN, M. G. ET AL. [ICECUBE-GEN2 COLLABORATION]. Icecubegen2: A vision for the future of neutrino astronomy in antarctica. 2014.

[173] AARTSEN, M. G. ET AL. [ICECUBE COLLABORATION]. Neutrino astronomy with the next generation icecube neutrino observatory. 2019.

[174] ADRIÁN-MARTÍNEZ, S. ET AL.. Letter of intent for km3net 2.0. J. Phys. G: Nucl. Part. Phys., 43:084001, 2016.

[175] HARDING, J. P. (ON BEHALF OF THE HAWC COLLABORATION). Constraints on the diffuse gamma-ray background with hawc. In: 36TH INTERNATIONAL COSMIC RAY CONFERENCE-ICRC2019, p. 1-6, Madison, WI, U.S.A., July 24th-August 1st, 2019.

[176] BAI, X. ET AL.. The large high altitude air shower observatory (lhaaso) science white paper. 2019.

[177] NERONOV, A.; KACHELRIESS, M.; SEMIKOZ, D. V.. Multimessenger gamma-ray counterpart of the icecube neutrino signal. Phys. Rev. D, 98:023004, 2018.

[178] CARROLL, S. M.. Spacetime and Geometry: An Introduction to General Relativity, chapter 8. Cosmology. Pearson Education, Inc., 2004.

[179] HARTLE, J. B.. Gravity: An Introduction to Einstein's General Relativity, chapter 18. Cosmological Models. Pearson Education, Inc., 2003.

[180] PLANCK COLLABORATION. Planck 2018 results. vi. cosmological parameters. arXiv:1807.06209v2 [astro-ph.CO], 2019.

[181] NTELIS, P.. The homogeneity scale of the universe. arXiv:1607.03418 [astro-ph.CO], 2016.

[182] SARKAR, S.; PANDEY, B.; KHATRI, R.. Testing isotropy in the universe using photometric and spectroscopic data from the sdss. arXiv:1810.07410v2 [astro-ph.CO], 2018. 
[183] RIESS, A, G.; CASERTANO, S.; YUAN, W.; MACRI, L. A.; SCOLNIC, D.. Large magellanic cloud cepheid standards provide a $1 \%$ foundation for the determination of the hubble constant and stronger evidence for physics beyond $\lambda \mathbf{c d m}$. arXiv:1903.07603v2 [astro-ph.CO], 2019.

[184] GELMINI, G. B.; KUSENKO, A.; TAKHISTOV, V.. Hints of sterile neutrinos in recent measurements of the hubble parameter. arXiv:1906.10136v1 [astro-ph.CO], 2019.

[185] MARCHESE, L.. Friedmann equations: The newtonian derivation of friedmann equations and an introductory study of them as a dynamical system. Bachelor thesis in physics, University of Trento, Via Sommarive 14, 38123 Povo (TN), 2018.

[186] HOPKINS, A. M.; BEACOM, J. F.. On the normalization of the cosmic star formation history. Astrophys. J., 651:142-154, 2006.

[187] HOPKINS, A. M.. On the evolution of star-forming galaxies. Astrophys. J., 615:209-221, 2004.

[188] YUKSEL, H. ET AL.. Revealing the high-redshift star formation rate with gamma-ray bursts. Astrophys. J. Lett., 683:L000-L000, 2008.

[189] AJELLO, M. ET AL.. The cosmic evolution of fermi bl lacertae objects. Astrophys. J., 780:73:1-24, 2014.

[190] BEREZINSKY, V.; BLASI, P.. Ultrahigh energy neutrinos from population iii stars: Concept and constraints. Phys. Rev. D, 85:123003, 2012.

[191] MEBANE, R. H.; MIROCHA, J.; FURLANETTO, S. R.. The persistence of population iii star formation. Mon. Not. R. Astron. Soc., 479:4:4544—4559, 2018. 


\section{A Brief Review on Cosmology}

Let us begin by reviewing the necessary tools from Einstein's theory of General Relativity (GR) that will be extensively used throughout this chapter. Our main goal is to lay out the basic foundations of cosmology required to become comfortable with the terminology used in astrophysics. Having said that, this section has no intention on providing a complete overview of the subject; the reader is referred to GR textbooks for more details [178, 179].

We start from the Friedmann-Lemaitre-Robertson-Walker (FLRW) metric $^{1}$ in natural units,

$$
d s^{2}=-d t^{2}+a^{2}(t)\left[\frac{d r^{2}}{1-k r^{2}}+r^{2} d \Omega^{2}\right],
$$

where $a(t)$ is a dimensionless scale factor representing the relative distances between two points at any given time $\left(a\left(t_{0}\right)=1\right.$, where $t_{0}$ is the present age of the universe) and $k=+1,0,-1$ for closed, flat or open universes, respectively (measurements of spatial curvature are consistent with a flat geometry, so we will adopt $k=0)$ [180]. We can write A-1 as $d s^{2}=-d t^{2}+a^{2}(t) d \Sigma^{2}$, where the coordinates $(t, \boldsymbol{\Sigma})$ are called comoving coordinates. An observer at constant $\boldsymbol{\Sigma}$ is said to be "comoving" and sees an isotropic universe.

In fact, equation A-1 represents a spatially homogeneous and isotropic universe, allowed to expand or contract. Observations have shown us that our universe satisfies these assumptions at scales $\gtrsim 200 \mathrm{Mpc}$, confirming the cosmological principle [181, 182].

To characterize the rate of expansion of the universe, it is common to use the Hubble parameter

$$
H=\frac{\dot{a}}{a}=\frac{d(\ln a)}{d t} .
$$

It's value today is called the Hubble constant, $H_{0}$, which has units of inverse time, but is usually expressed in the rather unusual units of $\mathrm{km} \mathrm{s}^{-1} \mathrm{Mpc}^{-1}$. This is because $H_{0}$ was first introduced by Edwin Hubble to explain the relation

\footnotetext{
${ }^{1}$ It is sometimes called Friedmann-Robertson-Walker, Robertson-Walker, Friedmann-Lemaitre, or simply Friedmann metric. This equation was first derived in the 1920s by the Soviet mathematician Alexander Friedmann; given its almost 100 years of existence, it is rather (un)impressive that there is still no consensus in literature as to what it's name should be!
} 
between the recessional velocity of galaxies $v$ (typically expressed in $\mathrm{km} \mathrm{s}^{-1}$ ) and their distance to us $d$ (typically measured in Mpc), known as Hubble's Law:

$$
v=H_{0} d
$$

Nowadays, we know that Equation A-3 is not exact because $H$ is not constant, but it is approximately valid for nearby galaxies, as we will soon see. Typical cosmological scales are set by the Hubble length, $d_{H}=\mathrm{cH}_{0}^{-1}$, and the Hubble time, $t_{H}=H_{0}^{-1}$.

It is worth mentioning that, currently, there is a troubling discrepancy between the two main independent measurements of $H_{0}$. Observations of Cepheids (pulsating stars) by the Hubble Space Telescope (HST) yield $H_{0}=$ $74.03 \pm 1.42 \mathrm{~km} \mathrm{~s}^{-1} \mathrm{Mpc}^{-1}$ [183]. On the other hand, CMB and BAO data from Planck satellite have obtained a value of $H_{0}=67.4 \pm 0.5 \mathrm{~km} \mathrm{~s}^{-1} \mathrm{Mpc}^{-1}$ [180]. Although there are several proposals for resolving this tension (see [184], for example) it remains a critical open problem in cosmology. Throughout this work, we will adopt the value $H_{0}=70 \mathrm{~km} \mathrm{~s}^{-1} \mathrm{Mpc}^{-1}$, which is commonly done in literature (at the end of the day, our results are independent of $H_{0}$ ).

Moving on, our next goal is to understand the phenomenon of cosmological redshift, which is essential for this chapter. Suppose a distant source emits two light pulses at times $t_{e}$ and $t_{e}+\delta t_{e}$, which are observed at times $t_{o}$ and $t_{o}+\delta t_{o}$, respectively. In other words, the pulses are emitted with frequency $\omega_{e}=2 \pi / \delta t_{e}$ and detected with frequency $\omega_{o}=2 \pi / \delta t_{o}$. Choosing axes in which these pulses propagate radially, we can take $d \Omega=0$ in Equation A-1. Since photons follow lightlike trajectories $\left(d s^{2}=0\right)$, each pulse satisfies

$$
\frac{d t}{a(t)}=\frac{d r}{\sqrt{1-k r^{2}}}
$$

Integrating along the light ray trajectories, we notice that the radial part is equal for both pulses:

$$
\int_{t_{e}}^{t_{o}} \frac{d t}{a(t)}=\int_{r_{e}}^{r_{o}} \frac{d r}{\sqrt{1-k r^{2}}}=\int_{t_{e}+\delta t_{e}}^{t_{o}+\delta t_{o}} \frac{d t}{a(t)}
$$

If we rewrite the last integral as

we obtain

$$
\int_{t_{e}+\delta t_{e}}^{t_{o}+\delta t_{o}}=\int_{t_{e}}^{t_{o}}+\int_{t_{o}}^{t_{o}+\delta t_{o}}-\int_{t_{e}}^{t_{e}+\delta t_{e}},
$$

$$
\int_{t_{o}}^{t_{o}+\delta t_{o}} \frac{d t}{a(t)}=\int_{t_{e}}^{t_{e}+\delta t_{e}} \frac{d t}{a(t)} .
$$

For small $\delta t_{e}$ and $\delta t_{o}$ (the time interval between two successive maxima of a light ray, for example), this becomes 


$$
\frac{\delta t_{o}}{a\left(t_{o}\right)}=\frac{\delta t_{e}}{a\left(t_{e}\right)}
$$

which means, in terms of the wavelength of the light ray,

$$
\frac{\lambda_{o}}{\lambda_{e}}=\frac{a\left(t_{o}\right)}{a\left(t_{e}\right)} .
$$

Since the scale factor was smaller at the time of emission, this means the observer will see a photon with a longer wavelength (i.e. a smaller energy) due to the cosmological expansion of the universe.

The redshift $z$ is defined as the fractional change in wavelength:

$$
z=\frac{\lambda_{o}-\lambda_{e}}{\lambda_{e}}
$$

Therefore, if the observation is made today $\left(a\left(t_{o}\right)=1\right)$, A-9 and A-10 imply ${ }^{2}$

$$
1+z=\frac{1}{a\left(t_{e}\right)}
$$

Although redshift strictly means the change in a photon's wavelength, we can interpret the cosmological redshift as a measure of distance or time. Astrophysicists colloquially refer to "a source emitting photons which exhibit a redshift of $z$ when measured at the Earth" as simply "a source at redshift $z$ ". The higher the redshift, the further away the source is from us. Additionally, when we observe sources at high redshift, we are seeing them as they were a long time ago because of the time it takes for their photons to reach us. The higher the redshift, the further we are looking into the past. Infinite redshift corresponds to the Big Bang, while $z=0$ corresponds to the present time.

Equation A-11 is valuable to us because it allows us to express the evolution of the Hubble parameter $H$ in terms of the redshift $z$. To achieve this, we must take a look at one of the two Friedmann equations, which is a direct consequence of writing down Einstein's field equations for a perfect fluid in the FLRW metric:

$$
H^{2}=\frac{8 \pi G}{3} \rho-\frac{k}{a^{2}}+\frac{\Lambda}{3}=\frac{8 \pi G}{3} \sum_{i} \rho_{i} .
$$

Here, $\Lambda$ is the cosmological constant and $\rho$ is the energy density of the fluid. In fact, there are three components which contribute to the universe's total energy density: matter $\left(\rho_{m}\right)$, radiation $\left(\rho_{r}\right)$, and vacuum $\left(\rho_{\Lambda}\right)$. The first two are included in $\rho=\rho_{m}+\rho_{r}$, while the last is represented by the cosmological constant term: $\rho_{\Lambda}=\frac{\Lambda}{8 \pi G}$. There is also a term relative to curvature $\left(\rho_{k}=\frac{-3 k}{8 \pi G a^{2}}\right)$, which is not necessarily an energy density, but must be accounted for in non-flat universes. Each component has a different dependency on $a(t)$

${ }^{2}$ Although all of these calculations were made assuming lightlike trajectories, this is still valid for particles with small masses, since the next-order term is proportional to $m^{2}$. Thus, we will adopt the same $(1+z)$ factor for the cosmological redshift of neutrinos. 
as $\rho_{i}=\rho_{i, 0} a^{-n_{i}}$. To find this dependency, one should propose an equation of state relating the fluid's energy density and pressure, and write down the conservation of energy using the energy-momentum tensor for a perfect fluid. For convenience, we only present the results in this dissertation (see Table A.1), and the reader is referred to [178], [179] or [185] for the details of these calculations.

Table A.1: Energy density dependence on the scale factor following $\rho_{i}=$ $\rho_{i, 0} a^{-n_{i}}$ for different components of our universe.

\begin{tabular}{c|c} 
& $n_{i}$ \\
\hline Matter & 3 \\
\hline Radiation & 4 \\
\hline Curvature & 2 \\
\hline Vacuum & 0
\end{tabular}

Defining the density parameters

$$
\Omega_{i}=\frac{8 \pi G}{3 H^{2}} \rho_{i}=\Omega_{i, 0} a^{-n_{i}}
$$

such that $\sum_{i} \Omega_{i}=1$, we can recall A-11 and finally write A-12 as

$$
H(z)=H_{0} \sqrt{\Omega_{m, 0}(1+z)^{3}+\Omega_{r, 0}(1+z)^{4}+\Omega_{k, 0}(1+z)^{2}+\Omega_{\Lambda, 0}},
$$

where $H(0)=H_{0}$ and we have made explicit all $z$-dependencies. Since our universe is (as far as we know) flat, we can take For our flat universe, we will take $\Omega_{k}=0$. Also, because the local universe is matter-dominated (rather than radiation dominated like the early universe), we can set $\Omega_{r, 0}=0$ and be left with the simpler expression

$$
H(z) \simeq H_{0} \sqrt{\Omega_{m, 0}(1+z)^{3}+\Omega_{\Lambda, 0}}
$$

which we will use throughout this work. Experimentally, we find that $\Omega_{m, 0} \simeq$ 0.3 and $\Omega_{\Lambda, 0} \simeq 0.7$. We will adopt these values in all our calculations.

Some final remarks must be made regarding distance measures in cosmology. "Distance" is a tricky concept in an expanding universe, and there exists several different notions of it. Nevertheless, all of them involve some sort of integration over FLRW spatial coordinates. It will be useful to express these integrals in terms of redshift instead, which can be easily done by using Equations A-2, A-4 for $k=0$ and A-11:

$$
\int_{0}^{r} \mathrm{~d} r=\int_{0}^{t} \frac{\mathrm{d} t}{a(t)}=\int_{a}^{1}=\frac{\mathrm{d}(\ln a)}{a H(a)}=-\int_{z}^{0} \frac{\mathrm{d} z^{\prime}}{H\left(z^{\prime}\right)}=\int_{0}^{z} \frac{\mathrm{d} z^{\prime}}{H\left(z^{\prime}\right)} .
$$


We have already introduced comoving coordinates in which distances are constant instead of evolving with $a(t)$. Associated to this coordinate system we have the comoving distance

$$
d_{c}(z)=\int_{0}^{z} \frac{\mathrm{d} z^{\prime}}{H\left(z^{\prime}\right)}
$$

in contrast with the proper/physical distance $d=a(t) d_{c}$. It is also common to talk about the comoving volume,

$$
\mathcal{V}(z)=\frac{4}{3} \pi d_{c}^{3}(z)
$$

which preserves number densities of non-evolving objects as the universe expands.

We can also measure distance by relating the radiated flux of objects to their luminosity. The source luminosity if defined as the photon energy emitted per unit time: $L=N E / \Delta t$, where $E$ is the energy of a single photon and $N$ is the total number of emitted photons. The flux we measure is the photon energy passing through a unit surface per unit time, which is related to the source luminosity by taking into account cosmological redshift and time dilation effects:

$$
F=\left(\frac{N E(1+z)^{-1}}{\Delta t(1+z)}\right)\left(\frac{1}{4 \pi d_{c}^{2}}\right)=\frac{L}{4 \pi d_{L}^{2}} .
$$

The associated distance is called luminosity distance,

$$
d_{L}(z)=(1+z) d_{c}=(1+z) \int_{0}^{z} \frac{\mathrm{d} z^{\prime}}{H\left(z^{\prime}\right)} .
$$




\section{SFR and other Source Population Distributions}

Two crucial calculations in this thesis are the neutrino and $\gamma$-ray propagation through intergalactic space (see Section 2.3 and Chapter 3, respectively, for details). Although they are qualitatively very different processes, both require the source population distribution as an input in order to obtain the total flux at the Earth. In other words, we must make an assumption for the number of sources per unit volume as a function of comoving coordinates, $\mathcal{F}(r, \theta, \phi)$ (units: $\left[\mathrm{Mpc}^{-3}\right]$ ). By assuming that these sources are extragalactic, isotropy at large scales ( $\gtrsim 200 \mathrm{Mpc}$, or equivalently, $z \gtrsim 0.05$ ) implies that $\mathcal{F}$ is solely redshift-dependent: $\mathcal{F}=\mathcal{F}(z)$.

This function can have radically different shapes depending on which astrophysical objects we are dealing with. In this work, we consider sources distributed according to the cosmic star formation rate $(\mathrm{SFR})^{1}, \dot{\rho}_{*}(z)$ (units: $\left.\left[\mathrm{M}_{\odot} \mathrm{yr}^{-1} \mathrm{Mpc}^{-3}\right]\right)$. This is an adequate assumption to make since starforming regions are likely to harbor dense environments where protons can be accelerated to ultra-high energies, producing $\mathrm{TeV}-\mathrm{PeV}$ secondary neutrinos and photons. This is the case for SBGs and GRBs, for example.

Multi-wavelength surveys across the entire electromagnetic spectrum have allowed us to model the SFR with a $\sim 30-50 \%$ accuracy up to $z \approx 1$ and within a factor of $\sim 3$ for $1 \lesssim z \lesssim 6[186,187]$. GRB observations have also allowed us to determine the SFR up to $z \approx 7$, with better precision [188]. We use the fit from [188] parametrized by a continuous doubly-broken power-law

$$
\dot{\rho}_{*}(z)=\dot{\rho}_{0}\left[(1+z)^{a \eta}+\left(\frac{1+z}{B}\right)^{b \eta}+\left(\frac{1+z}{C}\right)^{c \eta}\right]^{1 / \eta},
$$

where $a=3.4, b=-0.3$ and $c=-3.5$. The constants $B \simeq 5000$ and $C \simeq 9$ correspond to spectral breaks at $z \simeq 1$ and $z \simeq 4$, respectively, and $\eta=-10$ smooths the transition between the breaks $(\eta \rightarrow \infty$ recovers their sharpness). Figure B.1 explicitly shows this SFR fit for a normalization $\dot{\rho}_{0}=0.02 \mathrm{M}_{\odot} \mathrm{yr}^{-1} \mathrm{Mpc}^{-3}$. Our assumed comoving space density has the same shape, $\mathcal{F}(z) \propto \dot{\rho}_{*}(z)$, with its own normalization $\mathcal{F}_{0}$, which our analysis is

\footnotetext{
${ }^{1}$ Technically, the SFR is the rate of stellar mass formation (typically inside a galaxy). The correct term for the density distribution of star formation in space and time is "star formation history". For simplicity, we use SFR to refer to to both concepts in this thesis.
} 
blind to (See Section 4.2.3).

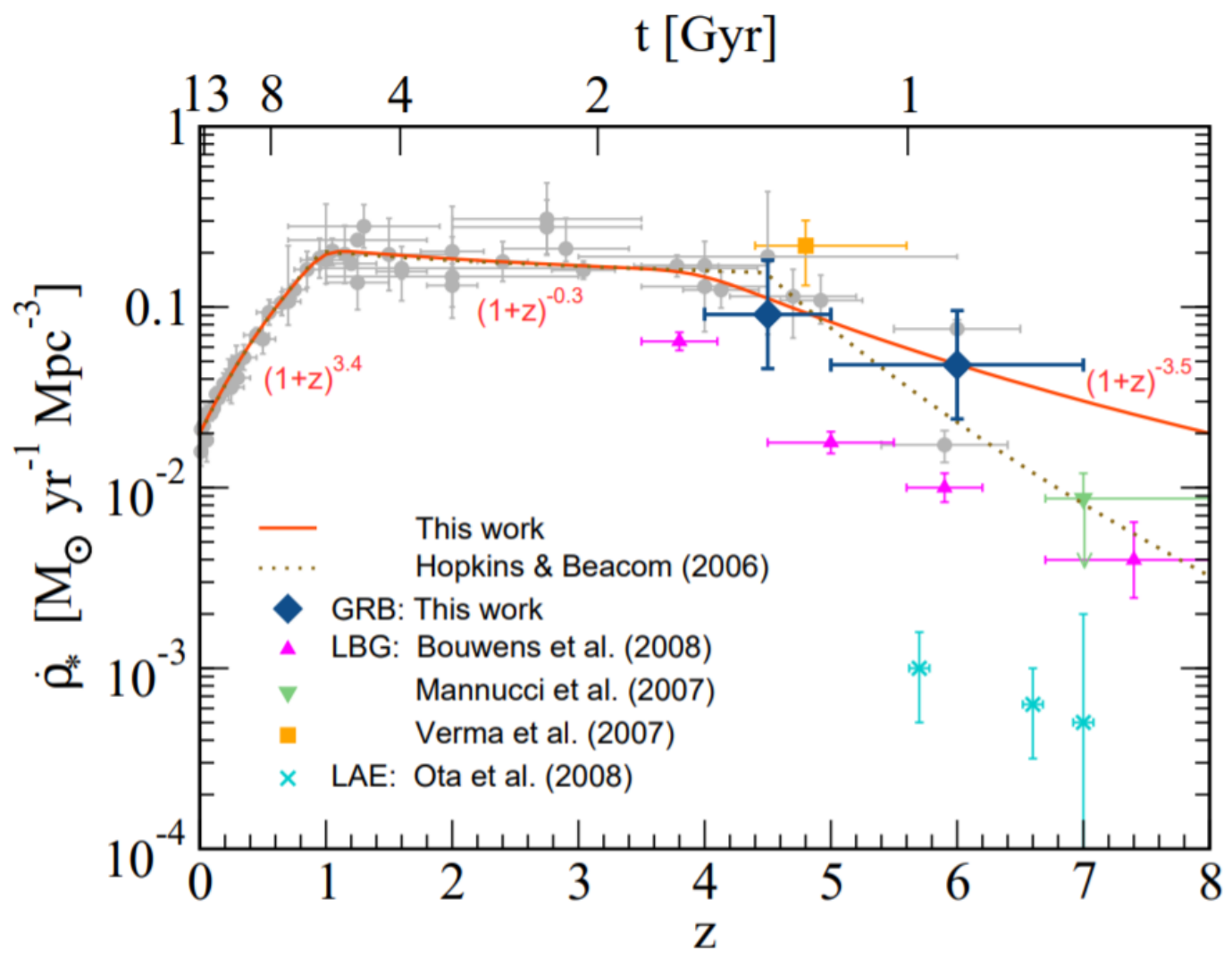

Figure B.1: Redshift dependence of the cosmic SFR density. The fit from Eq. B-1 is shown by the orange line. The gray points and dotted line are the compiled data and fit from [186]. The colored points are more recent high- $z$ data from this Figure's source article [188] and references therein.

BL Lacs are an example of candidate sources following a very different cosmic evolution than the SFR. We can obtain $\mathcal{F}(z)$ for BL Lacs by integrating their luminosity function (number density as a function of redshift, luminosity $L_{\gamma}$ and spectral index $\left.\Gamma\right)$ :

$$
\mathcal{F}_{\mathrm{BLL}}(z)=\int_{\Gamma_{\min }}^{\Gamma_{\max }} \int_{L_{\gamma, \min }}^{L_{\gamma, \max }} \operatorname{LF}\left(z, L_{\gamma}, \Gamma\right) \mathrm{d} L_{\gamma} \mathrm{d} \Gamma .
$$

$\operatorname{LF}\left(z, L_{\gamma}, \Gamma\right)$ has been modeled using Fermi data of $>200$ BL Lacs [189]. Figure B.2 shows both the SFR and BL Lac distributions for comparison. While the SFR dominates between $1 \lesssim z \lesssim 4$, leading to a fully developed $\gamma$-ray cascade for a large fraction of sources, the BL Lacs have peak density at $z=0$. This clearly reflects on the cascaded flux and, consequently, on the results of our analysis: while SFR-distributed sources peak at $\sim 100 \mathrm{GeV}$ and results in tension with the EGB data between 10 and $100 \mathrm{GeV}$, the BL Lac flux is shifted to higher energies and presents tension with the last $\sim 2$ EGB data points (see Figure 6 in [80]). 


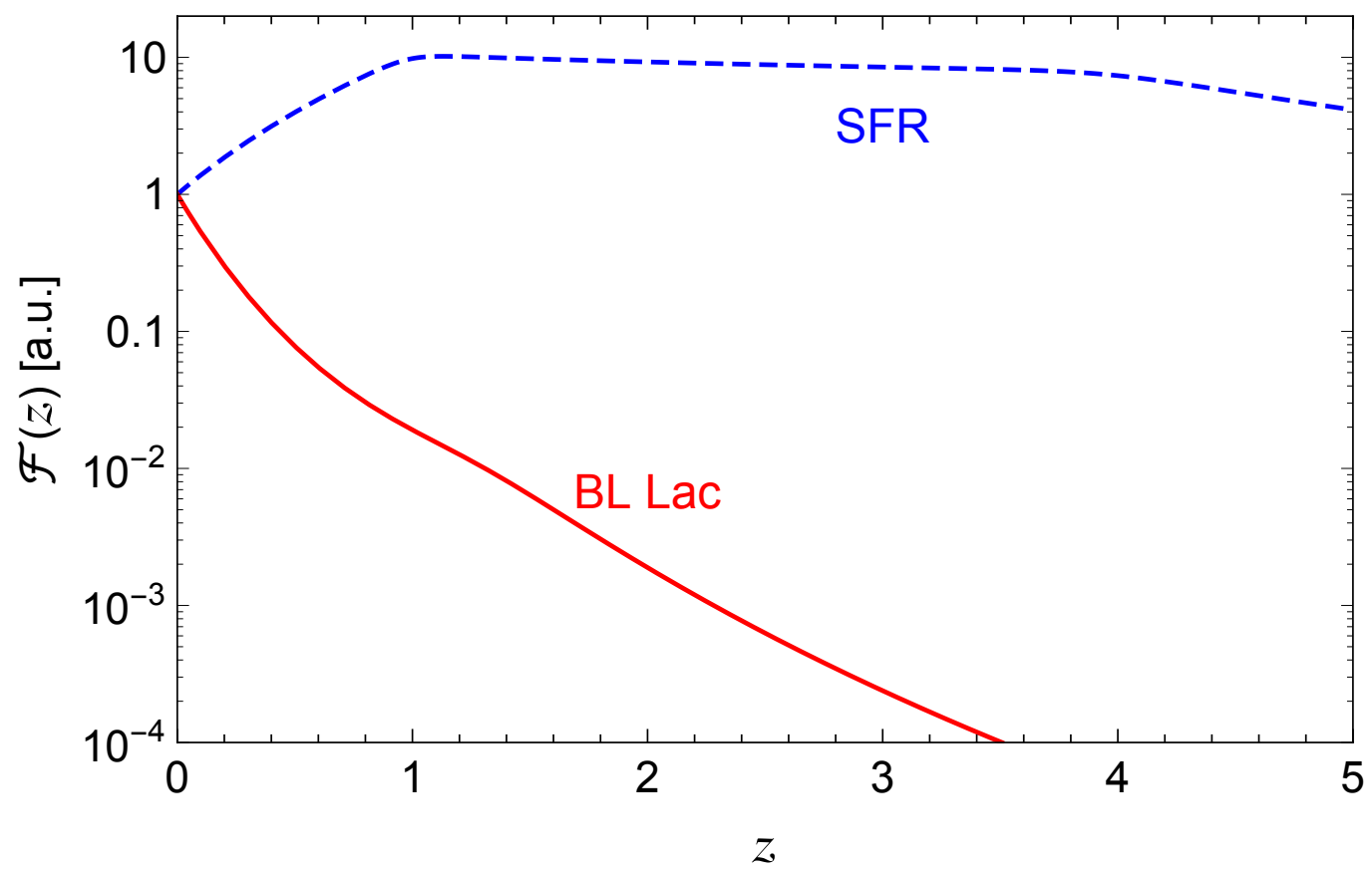

Figure B.2: Comparison between the SFR [188] and BL Lac [189] evolution functions normalized to unity at $z=0$. The BL Lac function has been parametrized using a luminosity-dependent density evolution model. Figure reproduced from [80].

In contrast, a high- $z$ class of objects which has been associated to the production of ultra-high energy neutrinos is Population III stars [190]. Their cosmic evolution has been modelled to extend from $z \approx 13$ up to $z \approx 45$ (see Figure 3 in [191]), which surely guarantees that their $\gamma$-rays have undergone the full electromagnetic cascade. Nevertheless, [80] has shown us that, although the tension with EGB data can be seemingly avoided for populations confined to $z \gtrsim 3$ with $E_{\mathrm{br}}=10 \mathrm{TeV}$, achieving the required neutrino luminosity budget would be extremely challenging, to put it lightly. 